\title{
THE EFFECTIVENESS OF COGNITIVE PROCESSING THERAPY AND PROLONGED EXPOSURE IN THE DEPARTMENT OF VETERANS AFFAIRS
}

\author{
BY \\ (C) 2014 \\ BENJAMIN TODD RUTT, M.S.
}
Submitted to the graduate degree program in Psychology and Research in Education and the Graduate Faculty of the University of Kansas in partial fulfillment of the requirements for the degree of Doctor of Philosophy

Chairperson: Thomas S. Krieshok, Ph.D.

James W. Lichtenberg, Ph.D.

David M. Hansen, Ph.D.

Mary E. Oehlert, Ph.D.

Philip C. McKnight, Ph.D.

Date Defended: 05/13/2014 
The Dissertation Committee for Benjamin Todd Rutt

certifies that this is the approved version of the following dissertation:

\section{THE EFFECTIVENESS OF COGNITIVE PROCESSING THERAPY AND PROLONGED EXPOSURE IN THE DEPARTMENT OF VETERANS AFFAIRS}

Committee Chairperson: Thomas S. Krieshok, Ph.D.

Date approved: 05/13/2014

- ii- 


\begin{abstract}
Multiple randomized clinical trials have shown that cognitive processing therapy (CPT) and prolonged exposure $(\mathrm{PE})$ are evidence-based treatments for posttraumatic stress disorder (PTSD). However, additional research should verify the effectiveness of CPT and PE in conditions more reflective of clinical practice. The present study evaluated the effectiveness of CPT and PE at reducing veterans' self-reported PTSD symptoms as measured by total scores on the PTSD Checklist (PCL). This study involved a retrospective review of 2,030 charts. 750 veterans from 10 U.S. states who received CPT or PE in individual psychotherapy from the Veterans Health Administration were included in the study ( $N$ participants in CPT $=376 ; N$ participants in PE = 374). The study used multilevel modeling to evaluate the absolute and relative effectiveness of both treatments and to determine the relationship between patient-level factors and total PCL scores during treatment. Results showed that both CPT and PE were equally effective at reducing total PCL scores, but that the effectiveness of both therapies was reduced when compared to results obtained in randomized clinical trials. Patients who completed therapy reported significantly larger reductions on the PCL than patients who did not complete therapy. On average, patients who did not complete a full course of therapy reported little to no improvement on the PCL. Encouragingly, as a group these patients did not report an increase in the severity of their total PCL scores prior to dropping out from therapy. Patients who reported more severe total PCL scores at baseline experienced significantly larger reductions in PCL scores during therapy, but veterans at all levels of initial PTSD symptom severity benefited from treatment. Older veterans and Hispanic veterans had higher total PCL scores at baseline. However, veterans across all ages and three racial/ethnic groups (Caucasian, African American, Hispanic) experienced equivalent rates of symptom reduction. Therapist effects were small, but
\end{abstract}


statistically significant. The study discusses the significance of these findings in light of prior research on trauma-focused therapy, future directions for research, and implications for practice. 


\section{Acknowledgements}

This study would not have been possible without the generous support of many people who, in one way or another, helped me along in my journey toward completing this dissertation.

After graduating from the University of Rhode Island with a degree in marine biology, I experienced a high amount of career indecision. For a time, I considered entering the field of medicine. I am fortunate that Col. Leon Moores, M.D. invited me to shadow him and other physicians at Walter Reed Army Medical Center in Washington, D.C. Volunteering at Walter Reed was a major turning point in my life. It helped me decide that I belonged in the field of psychology and is why I became interested in working with veterans. My experiences at Walter Reed also sparked my interest in trauma recovery, which started me on the path to this dissertation.

Thank you to the members of my dissertation committee, whose assistance was vital in bringing this study about. Dr. David Hansen helped me frame my research questions and provided me with valuable methodological consultation. Dr. Mary Oehlert has been one of my mentors almost from the beginning of my graduate training. I feel fortunate that she took me under her wing and encouraged me to explore research opportunities with the VA. I am thankful for the training in research methods and clinical research that I received from Dr. Jim Lichtenberg. Jim helped me learn how to think critically about psychotherapy outcome research, which provided me with the foundation for this dissertation. Finally, Dr. Tom Krieshok, my advisor, has been a source of constant support throughout my time at the University of Kansas. He has guided me through the multiple hurdles of my graduate program, served as a mentor for my clinical work and research, and provided me with a calm voice when I needed it most. 
The statistical and methodological consultation I received from Dr. Vicki Peyton was invaluable in helping me complete this study. She truly is the sixth member of my dissertation committee. Thank you to Dr. Kathy Copley, who helped fund this study through the CopwoodHill Dissertation Award. Thank you to Michael Carney, Richard Do, and Sundaragandhi Radhakrishnan for their logistical support. Finally, the training and mentorship I received from the CPSY faculty and the support I received from my fellow students were vital in enabling me to complete this study. I feel fortunate to have pursued my graduate training in such an enriching environment.

None of this would have been possible without my family. I inherited my passion and courage from my mother. When I was a child, she knew I would go far and gave me the freedom and confidence to follow my heart wherever it led. She also taught me to never forget to celebrate the milestones along the way. The completion of this study is certainly a milestone in need of celebration! I inherited my curiosity and calm demeanor from my father. From an early age, he helped spark my curiosity about the world, my love of learning, and taught me to ask questions. I have felt grounded in the calmness he instilled in me during times of crisis and during my development as a therapist. My sister has been a source of inspiration to me throughout my life. She has served as a role model for me and helped me decide to enter the field of psychology.

Last and most of all, words cannot express how thankful I am for the support my wife, Lindsay, has given me. I couldn't have done this without her. I am excited to complete this phase of my graduate training and am looking forward to beginning the next chapter in our life together, one in which we will have more time for each other and for the things in life that are most important to us. 


\section{TABLE OF CONTENTS}

Page

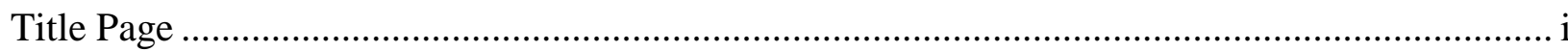

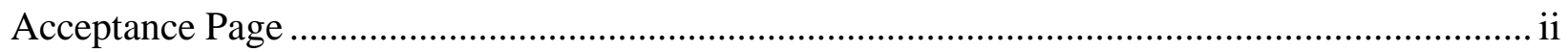

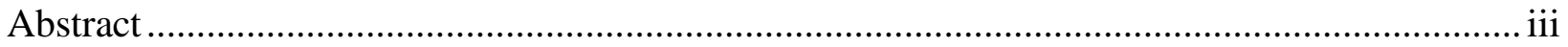

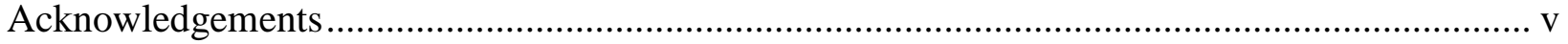

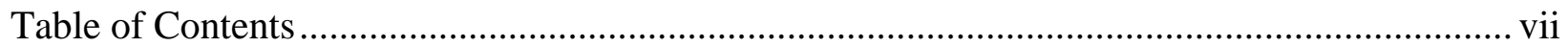

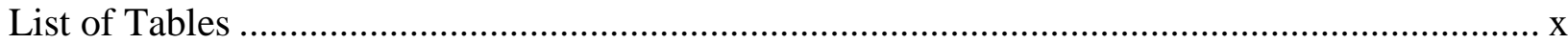

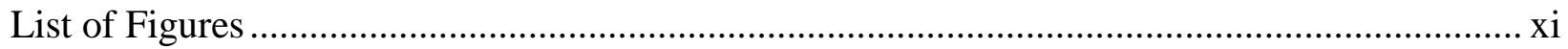

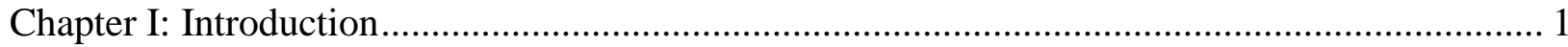

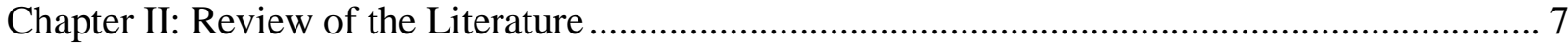

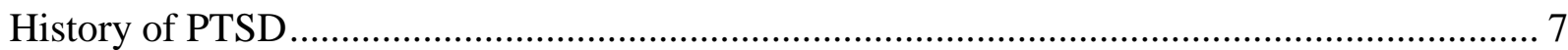

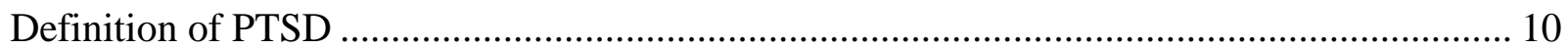

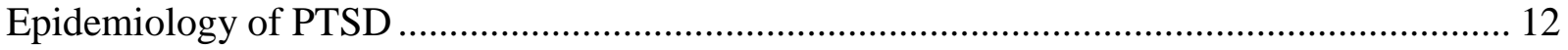

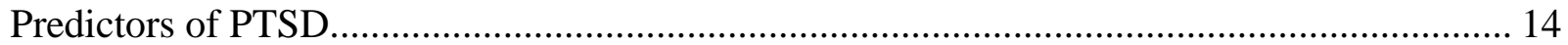

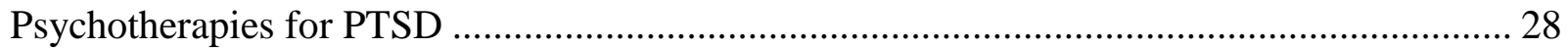

Factors Associated With Patient Outcomes ........................................................................ 35

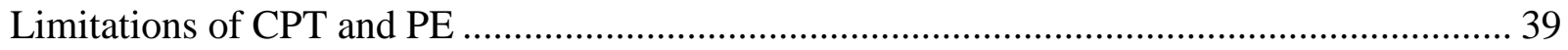

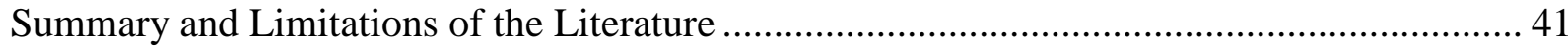

Research Questions \& Hypotheses ........................................................................... 43

-vii- 


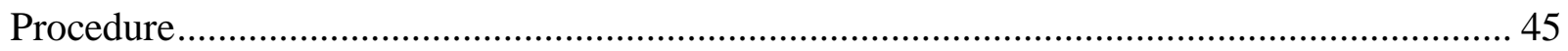

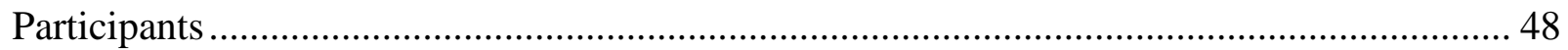

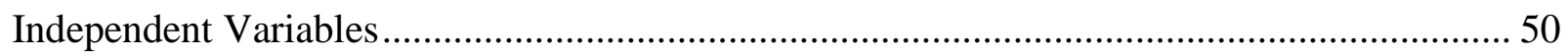

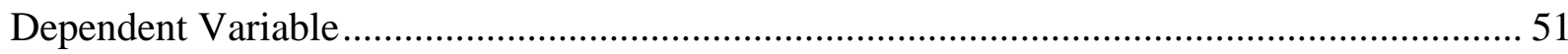

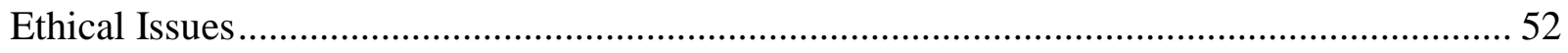

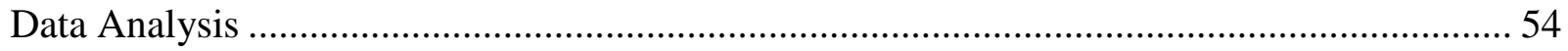

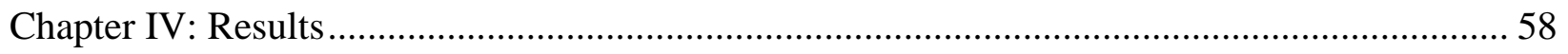

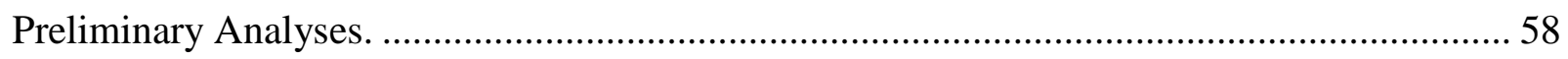

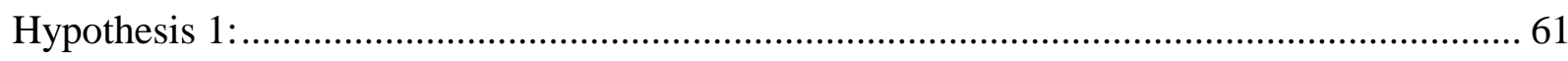

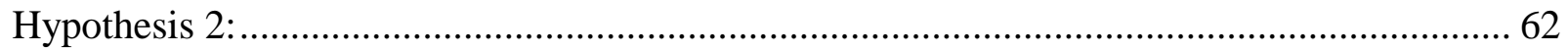

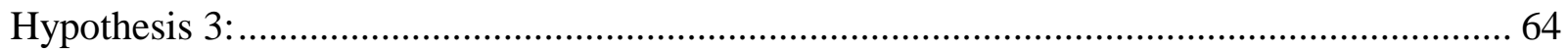

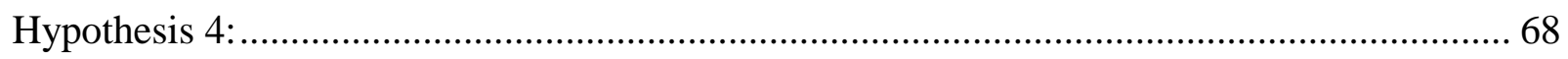

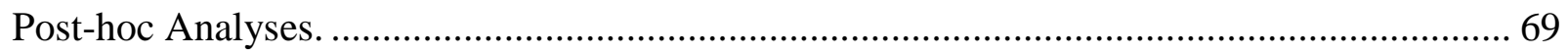

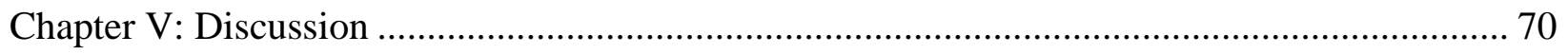

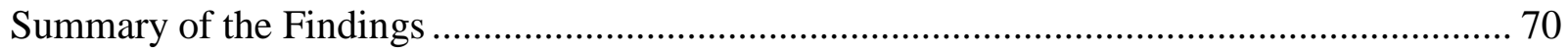

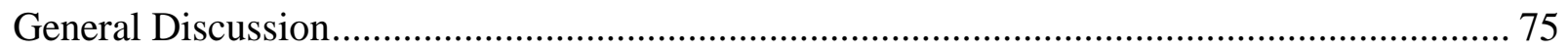

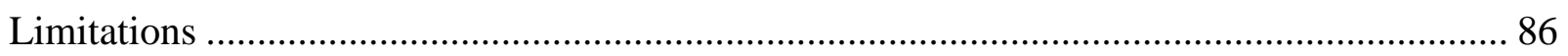

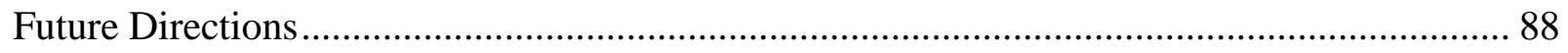

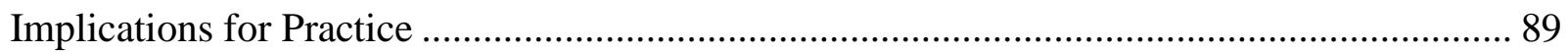


Conclusion.

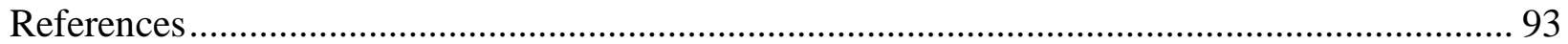

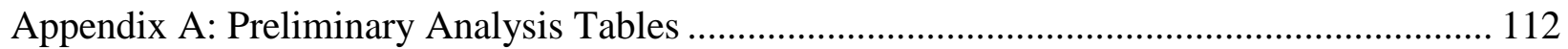

Appendix B: Results from Preliminary Analyses ............................................................. 115

Appendix C: Post-hoc Analysis of Therapist Effects ..................................................... 118

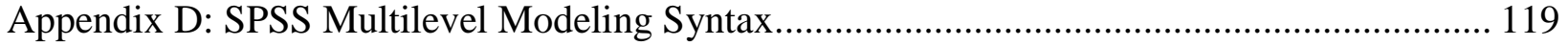

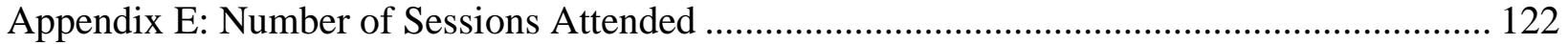




\section{LIST OF TABLES}

Page

Table 1. Demographics of sample and descriptive statistics. $(N=750)$

Table 3. One-way random effect ANOVAs for the three-level and two-level hierarchical models. 112

Table 5. Testing for random effects with the linear and quadratic slope terms. 114

Table 6. Frequency distribution of number of sessions attended for cognitive processing therapy $(C P T)$ and prolonged exposure (PE). 122 


\section{LIST OF FIGURES}

Page

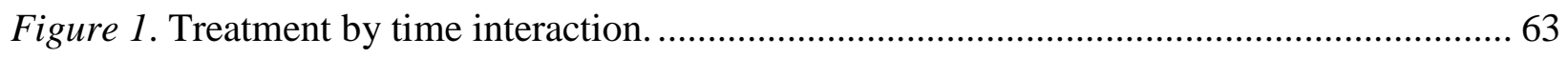

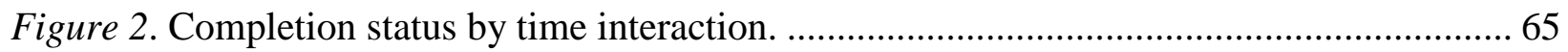

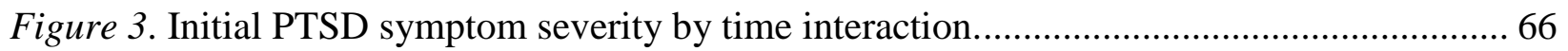




\section{Chapter I}

\section{Introduction}

"One cannot approach a veteran of prolonged, heavy combat from the position of knowing anything, other than one's own mortality and finitude." (Shay, 1991, p. 577).

The wars in Iraq and Afghanistan have become defining events in the lives of a generation of men and women who serve in the armed forces. Both wars have defined a new era in American history following the events of September 11, 2001. Operation Enduring Freedom (OEF) (2001 to present) began with the objectives of capturing Osama Bin Laden, dismantling Al Qaeda, and removing the Taliban from power. It has become the longest continuous deployment of U.S. ground forces. Operation Iraqi Freedom (OIF) (2003-2011) began with the objectives of finding weapons of mass destruction and deposing Saddam Hussein. However, it transitioned into an extended nation-building and counterterrorism operation. In 2010, the Department of Defense transitioned these activities into Operation New Dawn (OND).

Due to the protracted nature of the conflicts in Iraq and Afghanistan, nearly 2.2 million U.S. military personnel have served during OEF-OIF-OND (Institute of Medicine, 2013). Many veterans have returned from OEF-OIF-OND with deployment-related mental health problems. For those who have been exposed to combat in Iraq and Afghanistan, nearly 15 percent have developed posttraumatic stress disorder (PTSD) (Sundin, Fear, Iversen, Rona, \& Wessely, 2010). An even higher percentage of veterans may experience partial PTSD symptoms. Furthermore, approximately one third of veterans presenting for treatment served in multiple deployments (Seal et al., 2009), which increases the risk for PTSD (Hoge, Terhakopian, Castro, Messer, \& 
Engel, 2007). There also continues to be large cohorts of veterans from the Vietnam War and Operation Desert Storm who are in need of treatment for PTSD.

PTSD has been associated with a number of harmful outcomes, including death. The rate of completed suicides by veterans increased by 26 percent from 2005 to 2007, and has continued to grow (APA, 2013). Suicide also continues to be a great problem in the U.S. military. In 2012, an all-time high of 350 active duty members of the military committed suicide - more than the number of personnel killed in active duty in Afghanistan during that year (Dao \& Lehren, 2013). Increased prevalence of PTSD is one reason why suicides have increased during the last decade. PTSD also increases the risk of poor physical health (Norman et al., 2006), additional mental health problems (Kessler, Sonnega, Bromet, Hughes, \& Nelson, 1995), and substance abuse (Zatzick et al., 1997). People with PTSD are also more likely to be unemployed or underemployed, homeless, and divorced (Schnurr, Lunney, Bovin, \& Marx, 2009). Studies of PTSD clearly show that it has a negative impact on quality of life including reduced overall satisfaction with life, problems with interpersonal functioning, and vocational problems including reduced productivity and decreased vocational satisfaction ( Schnurr et al., 2009).

Given the many problems that veterans with PTSD experience, there is a great need for effective PTSD treatments. The present study addressed this need by researching the effectiveness of two evidence-based treatments for PTSD in the Veterans Health Affairs (VA) system.

The VA currently serves nearly 4 million veterans in hundreds of facilities across the United States (U.S. Medicine, 2009). To meet the needs of a large influx of OEF-OIF-OND veterans in recent years, the VA system recently implemented an initiative to research and disseminate evidence-based treatments for PTSD. The field of psychology has an important role 
to play in this national effort. Psychologists should continue to research and disseminate effective psychotherapies to treat PTSD so that this large cohort of veterans can receive the care that they need. Two treatments that have received a high amount of promotion in the VA are Cognitive Processing Therapy (CPT) and Prolonged Exposure (PE).

CPT adapts principles from Cognitive Behavioral Therapy for the treatment of PTSD. It involves creating a detailed account of the trauma, exploring the veteran's thoughts and feelings related to the trauma, and having veterans come up with more adaptive ways to process the trauma. PE is a behavioral treatment that encourages veterans to re-experience memories of traumatic events so that habituation of the anxiety response may occur. PE also encourages veterans to resume activities considered to be reasonably safe they previously avoided due to extreme anxiety (e.g., going to a restaurant; going to a public sporting event). Most veterans who participate in PE eventually realize that the emotional intensity related to their memories of the trauma as well as participating in public events decrease to manageable levels with repeated exposures. Both treatments involve creating a safe environment where veterans can feel comfortable as they talk about and work through their reactions to traumatic events. Due to their mutual focus on the traumatic events experienced by veterans, both therapies are often referred to as trauma-focused therapies.

Both CPT and PE have received much empirical support in the prior literature. Current findings from randomized clinical trials suggest that CPT and PE are equally efficacious (Benish, Imel, \& Wampold, 2008). The meta-analytic findings regarding CPT and PE have shown that patients who complete CPT or PE experience decreases in PTSD symptoms (Bradley, Greene, Russ, Dutra, \& Westen, 2005). However, such studies have largely been conducted in highly controlled settings. A smaller body of work (e.g., Chard et al., 2012; Jeffreys et al., 2014) has 
attempted to show the effectiveness of PTSD in settings that are more reflective of actual clinical practice.

The current study added to that body of literature by evaluating the effectiveness of CPT and PE within the real world setting of the VA system. The past research on CPT and PE has tended to focus on maximizing internal validity. This practice makes sense when a treatment is first being investigated. However, CPT and PE have both passed the initial review process with a high amount of empirical support. Both therapies have become highly implemented treatments for PTSD across the VA system. At this time, the research on these treatments should shift to having a greater focus on external validity to address the limitations of past studies.

One limitation of the past literature involves generalizing findings from the small samples in randomized clinical trials to the large population of veterans who are currently receiving treatment. Many of the thousands of veterans participating in CPT or PE in the VA may not have met the inclusion criteria for the past clinical trials. Many veterans currently receiving CPT or PE may also differ in potentially important ways from the research samples, such as race/ethnicity. Racial/ethnic minority patients have tended to not be well represented in randomized clinical trials. Additional research on trauma-focused therapy should verify that these treatments are effective for all veterans.

There is conflicting evidence regarding the differential effectiveness of treatments across different races/ethnicities. Some studies have found no significant effects of race/ethnicity (Alvarez, 2011) while others have (Walling, Suvak, Howard, Taft, \& Murphy, 2012). For those patients who fully participate, there have not been any observed differences in treatment outcomes across multiple racial/ethnic groups (Miranda et al., 2005). However, past research has tended to not investigate whether CPT and PE have a different level of effectiveness across 
multiple racial/ethnic groups. The current study addressed this limitation by evaluating the effectiveness of CPT and PE across three ethnicities: Caucasians, African Americans, and Hispanics.

The literature on CPT and PE has also suggested that certain groups of veterans respond differently to therapy. For example, there is evidence that veterans from the Vietnam War are less responsive to trauma-focused therapy than OEF-OIF veterans (Chard, Schumm, Owens, \& Cottingham, 2010). Other studies have suggested that veterans with more severe PTSD symptoms may experience a significantly larger reduction in PTSD symptoms (e.g., Jeffreys et al., 2014). The study included additional patient variables such as age and initial PTSD symptom severity to add to this body of literature. It is important to note that age may not be directly related to treatment outcome. Variables other than age, such as the type of combat exposure, may be more significantly related to treatment outcomes. However, age is one proxy variable that may enable the uncovering of this effect.

Past studies on CPT and PE have tended to have relatively small samples (e.g., below 150 participants) and were conducted in a relatively few number of treatment locations. Furthermore, most of the past research has utilized a pre-post design, which provides no information on how PTSD symptoms change between the first and last session. An additional limitation of pre-post designs is that there is no way to measure outcomes for patients who drop out from therapy. The current study addressed these limitations by including a large sample of patients from multiple treatment sites, by modeling self-reported PTSD symptoms from the beginning to the end of treatment, and by comparing outcomes between patients who complete treatment to those who dropped out from therapy. 
The researcher conducted a retrospective chart review of veterans who received CPT and PE. The study analyzed the trajectory of self-reported PTSD symptoms from a large, diverse sample of 750 veterans who received CPT or PE from VA facilities across the United States. The data from this study were analyzed using multilevel modeling (MLM). This approach involved the creation of a two-level hierarchical model: repeated measures over time (level 1) nested within individual patients (level 2). MLM enabled the researcher to model the change in PTSD symptoms over the course of treatment. The study sought to evaluate the absolute and relative effectiveness of each treatment, as well as which patient variables (i.e., completion status, initial PTSD symptom severity, age, and racial/ethnic status) are related to the reduction in PTSD symptoms.

Some researchers have suggested that therapist effects should be accounted for in psychotherapy outcome research and that ignoring therapist effects leads to biased estimates of treatment effects (e.g., Wampold, 2001). However, therapist effects are typically not accounted for in psychotherapy outcome research. In a post-hoc analysis, the current study calculated the size of therapist effects in the present sample to determine whether ignoring this variable biased the results.

The next chapter provides a brief history of PTSD, and then discusses the definition of the diagnosis as well as the epidemiology of the disorder. The chapter also reviews the risk factors for the disorder and potentially important variables that have received little attention to date in the literature. 


\section{Chapter II}

\section{Review of the Literature}

The current chapter begins with a description of the history of PTSD. It gives a brief overview of the current diagnostic criteria and then reviews the epidemiology of the disorder including its prevalence, comorbidity, chronicity, and clinical significance. A substantial portion of the chapter focuses on predictors of PTSD. The chapter then covers the treatments for PTSD. The section covering PTSD treatments begins with a description of the main meta-analytic findings. Then, it reviews the main findings in the outcome research of CPT and PE due to their prominence within the VA system. Following this section, the influence of race and ethnicity on psychotherapy outcomes and PTSD treatment outcomes in particular is reviewed. The chapter then gives a brief overview of therapist effects as a relatively unaccounted for variable in outcome research. The chapter concludes with a summary of the main findings and limitations of the literature and a brief description of the current study.

\section{History of PTSD}

The cluster of symptoms currently recognized as PTSD has been described in oral traditions, historical accounts, and literature for thousands of years (Birmes, Hatton, Brunet, \& Schmitt, 2003). Shay (1991) observed that there are many similarities between the experiences of Achilles in Homer's Illiad (850 BCE) and those of Vietnam veterans. Though separated by thousands of years, both accounts commonly involve a sense that war results in moral violations, as well as psychological reactions that include emotional numbing, intense grief following the loss of comrades, and misplaced guilt. Froissart (1334-1404 CE) described how dreams of past battles caused one medieval knight to rise from his bed and grab his sword on many occasions (cited in Birmes et al. 2003). The knight would swing the sword at his family members while in a 
semiconscious state, presumably in an attempt to fight off other soldiers in his dreams. Likening the traumatic reactions of people across historical eras to our modern understanding of PTSD is tenuous. It ignores the cultural context within which people experience traumatic reactions and relies on anecdotal accounts. However, such a comparison shows that some aspects of what is now considered PTSD have been commonly reported throughout human history.

Scientific inquiry into human reactions to trauma began in earnest during the $19^{\text {th }}$ Century (Lasiuk \& Hegadoren, 2006a). One of the earliest clinical descriptions of what would later become known as PTSD was in the 1860s following observations of people who survived railway accidents. Waller Lewis, a British physician, noted that many survivors of such accidents often experienced sleep disturbances, nightmares about collisions, and intolerance of railway travel. He classified this condition as railway spine. Other physicians who treated military veterans described similar reactions to the trauma of combat. Meyers (1870) observed that combat veterans often reported experiencing fatigue, palpitations, sweating, tremors, and fainting, a condition he called soldier's heart. Da Costa (1871) observed similar symptoms in veterans of the American Civil War, which later became known as Da Costa's syndrome. Within the psychiatric community, the above diagnoses were soon called traumatic neurosis or battle neurosis.

As traumatic neurosis became increasingly described, the medical community debated its etiology (Lasiuk \& Hegadoren, 2006a). At the heart of the matter was a central question regarding whether dispositional or environmental factors were responsible for the disorder. Some argued that traumatic neurosis was due to flawed personality traits and malingering, while others argued that it was due to exposure to traumatic events. An additional focus of the debate involved whether organic or psychiatric factors caused the disorders. Janet, a French psychiatrist, 
proposed that people with psychological trauma were incapable of integrating memories of the traumatic event and the intense emotions that became associated with them into their narrative memory (Lasiuk \& Hegadoren, 2006a). During the formative years of psychoanalysis, Freud and Bruer also found that enabling people to put into words their reactions and emotions surrounding past traumatic events led to a reduction in symptoms.

The issue of battle neurosis increased in prominence due to World War I. As the war progressed, many soldiers who experienced the horrors of trench warfare began experiencing severe psychological reactions to combat. Physicians treating these soldiers frequently described symptoms including screaming, uncontrollable crying, emotional numbing, irritability, traumatic dreams, and an exaggerated startle response. Meyers (1915), a British military psychiatrist, described these symptoms as being part of a new condition called shell shock. Although he originally thought the disorder was caused by organic damage due to a soldier's proximity to exploding shells, Meyers later recognized that shell shock also involved a psychological component. However, the majority of military authorities did not consider shell shock to be a legitimate medical condition. Instead, they considered soldiers with shell shock to be cowards and malingerers. Some soldiers with shell shock were even executed (Babington, 1993).

During World War II (WWII), shell shock was renamed combat fatigue or combat stress. At the time, the U.S. military implemented psychological screens in an attempt to remove from service individuals who were considered psychologically unfit (Lasiuk \& Hegadoren, 2006a). The emphasis during this period was still largely on how dispositional traits resulted in making some people unfit for service. Despite the U.S. military's efforts to weed out psychologically unfit soldiers, combat fatigue became one of the most common diagnoses in military hospitals during WWII. The prevailing opinion among military commanders was that combat fatigue was 
caused by dispositional weaknesses rather than environmental influences. However, as the war progressed military psychiatrists increasingly recognized that almost all soldiers were susceptible to psychiatric problems following prolonged combat (Atkinson, 2002). When veterans returned home following WWII, they rarely spoke about their experiences or reactions to the war. However, as cultural attitudes regarding mental illness shifted, the momentum to create a formal diagnosis for psychological reactions to trauma increased. This was in part due to yet another generation of returning combat veterans from the Vietnam War.

By 1980, the American Psychiatric Association formally recognized PTSD in the DSMIII as a diagnosable mental disorder. This development was not without controversy. There are still active debates regarding the validity and clinical utility of the PTSD diagnosis (Dell'Osso \& Carmassi, 2011). However, there is broad agreement that both individual and environmental variables act as risk factors of PTSD.

\section{Definition of PTSD}

Since 1980, the definition of PTSD has undergone multiple changes (Lasiuk \& Hegadoren, 2006b). Previously, PTSD was conceptualized as consisting of three clusters that included intrusive thoughts, avoidance, and hyper-arousal. However, studies within the last 20 years have indicated that four symptom clusters is a more accurate (Simms, Watson, \& Doebbeling, 2002). By using confirmatory factor analysis, Simms and others have shown that the fit statistics for a four-factor model of PTSD is significantly better than the three-factor model. Subsequently, the American Psychiatric Association altered the definition of PTSD to take this evidence into account. Recently, a five-factor model with dysphoric arousal specified as a potential new factor has also been proposed (Armour et al., 2012). However, the factor analytic research of PTSD is ongoing and subject to debate. 
In the DSM-V, PTSD is classified as a trauma and stressor-related disorder. Criterion A specifies that a person must be exposed to a traumatic event during which they experienced, witnessed, or were confronted with an event or events that involved actual or threatened death or serious injury, or a threat to the physical integrity of others. The person's response must involve fear, helplessness, or horror. PTSD is specified as consisting of four clusters of symptoms: (1) intrusive thoughts (2) avoidance (3) negative alterations in cognitions and mood, and (4) alterations in arousal and reactivity.

Intrusive thoughts may include recurrent and intrusive distressing recollections of the event, recurrent distressing dreams of the event, acting or feeling as if the traumatic event were recurring, intense psychological distress at exposure to cues that resemble the traumatic event, or physiological reactivity to cues that represent the event. Avoidance may include efforts to avoid thoughts, feelings, activities, or conversations associated with the trauma. It may also involve an inability to recall an important aspect of the trauma,

Negative alterations in cognition and mood can involve emotional numbing, diminished interest or participation in activities, feelings of detachment or estrangement from others, restricted range of affect, and a sense of a foreshortened future. Alterations in arousal and reactivity can include difficulty falling or staying asleep, irritability or outbursts of anger, difficulty concentrating, hyper-vigilance, exaggerated startle response, or reckless and destructive behavior. Individuals may also have persistent and distorted blame of self or others and a persistent negative emotional state.

A new clinical subtype "with dissociative symptoms" was also added for individuals for whom depersonalization and/or de-realization is especially prominent. 


\section{Epidemiology of PTSD}

Prevalence. Within the civilian population, the lifetime prevalence of PTSD is about 7.8 percent (Kessler et al., 1995). However, the prevalence of PTSD is significantly higher among military veterans and that prevalence varies from conflict to conflict. Vietnam veterans appear to have the highest prevalence of PTSD, with somewhere between 8.5 and 19.3 percent of veterans meeting criteria for PTSD (Magruder \& Yeager, 2009). In one of the largest studies of Vietnam Veterans, the National Vietnam Veterans Readjustment Study (NVVRS) estimated the prevalence of PTSD to be 15.2 percent (Kulka et al., 1990). However, Kulka et al. (1990) found that close to one-third of Vietnam Veterans had experienced PTSD at some point in time. Magruder and Yeager's (2009) meta-analysis estimated the prevalence of PTSD among Persian Gulf War veterans to between 1.9 and 24 percent. For OEF-OIF veterans, between 4.7 and 19.9 percent met criteria for PTSD. The wide range in estimates of the prevalence of PTSD across studies is largely due to a variation in methodology, as well as when PTSD was assessed (Sundin et al., 2010). Sundin et al. (2010) found that PTSD prevalence was higher in studies that used anonymous surveys and that PTSD symptom severity increased in the months following return from deployment. The prevalence of PTSD among veterans seeking treatment in the Veterans Affairs (VA) system is even higher, with one study estimating its prevalence to be near 22 percent (Seal et al., 2009).

Comorbidity and Chronicity. People who have PTSD commonly have comorbid diagnoses. Kessler et al. (1995) found that among people diagnosed with PTSD in the general population, nearly 90 percent had at least one other mental disorder at some point in time. They found that the most commonly diagnosed comorbid conditions were major depression, alcohol abuse, alcohol dependence, drug abuse, drug dependence, and additional anxiety disorders such 
as social phobia and generalized anxiety disorder. Within the VA system, the most common comorbid disorders include major depression, anxiety disorders, and substance abuse or dependence (Magruder et al., 2005). Magruder et al. (2005) also found the comorbidity rate of veterans with PTSD was similar to the civilian population.

The evidence suggests that PTSD often becomes a chronic disorder. In the general population, Kessler et al. (1995) found that nearly 30 percent of people with PTSD still had symptoms ten years following the onset of symptoms. In one study, WWII and Korean War veterans reported significant PTSD symptoms up to 50 years following their service (Spiro, Schnurr, \& Aldwin, 1994). Schnurr, Lunney, Sengupta, and Waelde (2003) found that the average symptom duration of PTSD among Vietnam veterans was 18 years. Without treatment, veterans of OEF-OIF who have PTSD are likely to continue experiencing symptoms long into the future. Furthermore, the severity of PTSD symptoms tends to increase over time, especially as patients become further isolated from sources of social support due to the illness.

Clinical Significance of PTSD. PTSD is associated with a number of negative behavioral and health outcomes. As noted above, the likelihood of substance abuse and dependence is greater among people who have PTSD (Kessler et al., 1995). Hoge et al. (2007) found that about one-quarter of Army soldiers and one-third of marines engage in hazardous drinking. Hazardous alcohol use is more common among younger veterans and men (Scott et al., 2013). Guerra and Calhoun (2011) found that, even after controlling for combat exposure and suicide attempt history, PTSD alone was associated with an increased risk of suicidal ideation. For patients with PTSD, Guerra and Calhoun (2011) found that suicidality was uniquely associated with emotional numbing symptoms. People with PTSD are also more likely to engage in violent behavior (Zatzick et al., 1997), including domestic violence. 
PTSD is associated with lower quality of life across a number of domains (Schnurr, Lunney, Bovin, \& Marx, 2009). Among OEF-OIF veterans, Schnurr et al. (2009) found that people with PTSD are more likely to be unemployed and homeless, have problems with social and interpersonal functioning, have strained relationships with family and at work, and be divorced. Furthermore, OEF-OIF veterans with PTSD are likely to report significantly lower overall life satisfaction. It also appears that the different clusters of PTSD symptoms may influence quality of life in unique ways (Shea, Vujanovic, Mansfield, Sevin, \& Liu, 2010). Shea et al. (2010) found that emotional numbing symptoms appear to be uniquely associated with decreased social and occupational functioning while hyper-arousal symptoms are associated with subjective ratings of distress and lower clinician ratings on the Global Assessment of Functioning scale.

PTSD has also been associated with numerous negative physical health outcomes. People with PTSD are at an increased risk for many physical health problems including digestive problems, problems with the nervous system, memory loss, fatigue, and knee and joint problems (Possemato, Wade, Andersen, \& Ouimette, 2010). The impact of PTSD on physical health can last for many decades (Boscarino, 1997).

\section{Predictors of PTSD}

The following section organizes the predictors of PTSD within the biopsychosocial model. The terms predictor and risk factor are used interchangeably throughout. Also, it should be noted that predictors of PTSD can interact with one another in complex ways. Wherever possible, such interactions are noted. The below section focuses on research conducted with veteran or members of the military. However, studies that were conducted with civilian samples that are highly relevant are also included. 
Exposure to Trauma. Predictors of PTSD range from internal characteristics to environmental influences. Of these influences, the most important predictor of PTSD is exposure to trauma. By definition, exposure to trauma must occur in order for PTSD to develop. Such trauma may be experienced directly or witnessed. All that is required is a real or perceived threat to life or a severe intrusion or violation of the body, as in sexual assault. In the civilian population, Kessler et al. (1995) found that rape and sexual molestation were the most common traumas associated with PTSD among women. In OEF-OIF veterans, military sexual assault has gained increasing attention in recent years as one type of trauma that disproportionally affects women (Institute of Medicine, 2013). For men, combat exposure and witnessing combat or death are the most common traumas associated with PTSD (Kessler et al., 1995). The occurrence of additional traumatic events following the initial trauma can increase the risk of subsequent PTSD (King, King, Vogt, Knight, \& Samper, 2006).

Biological Predispositions. The body responds to stress and trauma in a number of ways. Physiological mechanisms, such as the hypothalamic-pituitary-adrenal axis, changes in neurotransmitter activity, and the activity of hormones (e.g., cortisol) are all components of the stress response. There is strong evidence that individual differences in the stress response, as well as other biological factors, may increase the risk of PTSD. For example, studies have shown that mutations in genes that alter the activity of serotonin (Goenjian et al., 2012) and dopamine (Hoexter et al., 2012) are significantly more common in individuals with PTSD. Furthermore, a mutation in the glucocorticoid receptor is significantly more common in soldiers who developed PTSD following combat (Van Zuiden et al., 2012). The glucocorticoid receptor is found in almost every cell in the body and is one of the main receptors for cortisol, a key stress hormone. Interestingly, De Quervain et al. (2012) found that one mutation in a gene associated with 
memory capacity was found in survivors of the Rwandan genocide who were diagnosed with PTSD. Such a mutation may increase PTSD risk by making traumatic memories more accessible post-trauma.

Although genetic mutations can create biological predispositions for mental illness, there has been growing evidence that gene-environment interactions are a key factor in the development of PTSD (Mehta \& Binder, 2012). We now know that environmental factors can directly alter gene expression. For example, a traumatic event may increase the expression of a receptor gene, which would alter the binding activity of specific neurotransmitters or hormones to that cell. If those changes occur in multiple neurons, it could alter the pattern of neural activity in the brain. Thus, altering the expression of genes in the central nervous system could have important implications for the development of PTSD (Zovkic \& Sweatt, 2012).

Personality Traits. Following the development of personality tests, many researchers attempted to identify which personality traits predispose people to developing PTSD following exposure to traumatic events. For example, one recent study found that high PTSD symptoms among National Guard and Army Reserve service members were associated with higher neuroticism, lower conscientiousness, and lower openness to experience (Caska \& Renshaw, 2013). Caska and Renshaw also found evidence that extraversion moderates the association between severity of combat and PTSD symptom severity. However, a significant limitation of such studies is their cross-sectional design. Cross-sectional studies may uncover associations between personality traits and PTSD, but their design makes it impossible to determine the direction of the relationship. Although it is possible that some personality traits may predispose people to developing PTSD following trauma exposure, traumatic events may also be severe 
enough to alter a person's personality. Anecdotally, family members of people with PTSD often report large changes in the personality of significant others with PTSD.

Of the many studies that have attempted to link PTSD to personality factors within combat veterans, at least two involved the administration of personality measures prior to trauma exposure. One study involving WWII veterans found that the psychological vulnerability of soldiers before combat was associated with trait neuroticism four decades later (Lee, Vaillant, Torrey, \& Elder, 1995). The researchers measured personality traits immediately before or after deployment during WWII and then again in the early 1990s. However, there was no relationship between trait neuroticism and PTSD symptom severity. The reporting of trauma symptoms in this study's sample was completed in 1946, so it is possible that the severity of trauma symptoms reported by service members was artificially low due to the cultural attitudes regarding mental illness at the time. Schnurr, Friedman, and Rosenberg (1993) compared pre-combat MMPI scores obtained in the 1960s with PTSD symptoms measured in the late 1980s in a sample of Vietnam veterans. After controlling for the level of combat exposure, the investigators found that pre-combat scores on the psychopathic deviate, masculinity-femininity, social introversion, and paranoia scales were significantly more elevated in individuals who would later develop PTSD. Unfortunately, the aforementioned studies are rare and most studies on PTSD and personality traits are cross-sectional. An additional weakness of such studies is their tendency to place the blame for PTSD within the individual while ignoring the considerable influence of environmental factors on the expression of trauma symptoms.

Personal History. Multiple personal history variables have been investigated as risk factors for PTSD. Researchers have focused on psychological adjustment prior to trauma exposure, family history, and history of prior trauma among other variables. A meta-analysis of 
63 studies found that all three variables had a small but statistically significant effect size ( $r=$ .17) on trauma symptoms (Ozer, Best, Lipsey, \& Weiss, 2003). The large body of evidence suggests that while these variables play a role, other variables are more important predictors of PTSD.

Age is an additional personal variable that has also consistently been implicated as a predictor of PTSD, especially in soldiers and combat veterans. Brewin, Andrews, and Valentine (2000) found that military veterans who were younger age at time of trauma were at a significantly higher risk of developing PTSD. This finding has held up in additional studies of OEF-OIF veterans (Grieger et al., 2006).

Cognitive and Affective Factors. Given the large emphasis on researching environmental risk factors for PTSD (e.g. combat exposure), the role of cognitive and affective responses in the development of PTSD was unappreciated until relatively recently. Thoughts and emotions that occur during a traumatic event are referred to as peritraumatic responses. Much research has shown that peritraumatic responses, as well as the cognitive and affective responses of people post-trauma, are important factors in the development of PTSD. All environmental stimuli, including trauma, are filtered through a person's perceptual systems. Thus, it is understandable that the cognitive and affective reactions people have during and after trauma are important risk factors for PTSD. It seems reasonable that the impact of trauma is at the very least moderated by cognitive and affective factors. However, recent findings also suggest that, at least in some cases, cognitive and affective factors may directly mediate the impact of trauma on PTSD symptoms.

One meta-analysis showed that the effect of combat on PTSD is mediated by the perceived threat of combat experiences (Ozer et al., 2003). This implies that perceptions people 
have during combat regarding the level of threat is an important risk factor for PTSD. King, King, Gudanowski, and Vreven (1995) found that when people are passive recipients of trauma (i.e. being close to an IED blast, being shot at, etc.) their PTSD symptoms are mediated by the level of threat they perceive in that situation. As with other attributions, perceptions of threat can have varying levels of accuracy. Some forms of combat are highly threatening. In those situations, service members may accurately perceive that the level of threat is high and have an increased likelihood of PTSD.

However, the level of training and prior experience of service members can influence the accuracy of their perceptions of threat. Service members who received limited training may inaccurately inflate the level of perceived threat in some situations and subsequently be at an increased risk for PTSD. One study of OEF-OIF veterans found that although the level of combat exposure between Active Duty and Army Reserve and National Guard troops was similar, a higher percentage of Reserve and National guard troops reported the belief that they might be killed during combat (Milliken, Auchterlonie, \& Hoge, 2007). The Army Reserve and National Guard soldiers in this study also reported significantly more mental health problems, including PTSD.

Another study showed that the type of coping that soldiers used during and immediately following combat was significantly related to their risk for developing PTSD (Sharkansky et al., 2000). When faced with a trauma or a traumatic event, people have two broad types of coping they may engage in. Approach-based coping involves a direct attempt to resolve or manage the stressor. Avoidance-based coping involves an attempt to avoid thinking about or avoid experiencing the affect associated with the stressor (Moos, 1990). Sharkansky and colleagues found that Persian Gulf soldiers who used more approach-based coping were more likely to think 
about their experiences, make positive reappraisals of the situation, and seek out social support. Such soldiers had better outcomes than those who coped with combat stress by avoiding thinking about it or giving up. An additional key finding in this study was that approach-based coping was significantly associated with higher resources. Soldiers who received additional training prior to combat exposure due to active duty status, older soldiers, and officers were more likely to use approach-based coping.

There is increasing evidence that trauma-related guilt is an additional predictor of PTSD (Kubany et al., 1995). Trauma-related guilt, as defined by Kubany and colleagues (1995), involves both cognitive and affective components. Individuals who feel trauma-related guilt may perceive that they did something wrong or violated a moral principal. Often, they perceive that they were the cause of the negative event, whether due to action or inaction. If there is a perceived lack of justification for their actions (e.g. killing a civilian), guilt is often more intense. Furthermore, individuals with trauma-related guilt often engage in hindsight bias- the person believes he or she should have foreseen the traumatic event. Depending on the situation, while such thoughts may be accurate, they can also be debilitating. The affective component of traumarelated guilt involves intense feelings of distress due to a traumatic event. Kubany et al. (1995) found that the most commonly reported sources of guilt among Vietnam combat veterans were perceptions of not being able to do more during combat. Related to this concern was the perceived inability of veterans to prevent the death or suffering of civilians or fellow American soldiers. Furthermore, accidental harm to others and the accidental killing of civilians were reported by 12 percent of veterans in their sample. Given that their study occurred twenty to thirty years after the Vietnam War, it is significant that trauma-related guilt was highly related to PTSD symptoms decades after the traumatic events occurred. 
Emotional factors are also relevant components in the traumatic response. During the peritraumatic emotional response, it is common for people to feel intense horror, fear, and helplessness. Ozer et al. (2003) found that the peritraumatic emotional response exerted a significant $(r=.35)$ effect on subsequent PTSD symptoms.

Peritraumatic Dissociation. Given the intensely distressful emotions and cognitions that arise during and after traumatic events, it is a common response for many people is to distance themselves from such experiences. Peritraumatic dissociation involves the detachment and distancing from unpleasant emotions and thoughts during a traumatic event. Individuals who use this coping strategy often report experiencing a sense that the traumatic event was somehow unreal. Peritraumatic dissociation is a response to severe trauma that protects people from horror. Although it may lead to some short-term benefits during traumatic events (e.g., emotional distance from horrifying events), it is actually one of the most important risk factors for subsequent PTSD (Marmar, Weiss, Schlenger, Fairbank, \& et al., 1994).

In Ozer et al.'s (2003) meta-analysis, peritraumatic dissociation was the strongest predictor of PTSD. They concluded that people who have dissociative experiences during or immediately after the traumatic event have a significantly higher level of PTSD symptoms. Peritraumatic dissociation is also a significant factor in the persistence of PTSD (Schnurr, Lunney, \& Sengupta, 2004). Recent changes in the diagnostic criteria in the DSM-V have taken these findings into account, creating a dissociative subtype of PTSD for when such symptoms are particularly prominent.

Life Stress. Life stress has been implicated as an additional risk factor for PTSD (Brewin et al., 2000). Simply living in a war zone can increase the risk of PTSD (King et al., 1995). King et al. (1995) refer to war zones as malevolent environments—environments marked by repeated 
day-to-day irritations and aggravating living conditions. Malevolent environments result in people feeling increased levels of distress, as well as feelings of helplessness, futility, and emotional emptiness. A meta-analysis of PTSD symptoms experienced by veterans of WWII through OEF-OIF found that simply being deployed to a war zone results in a 1.5 to 3.5 -fold increase in PTSD risk, regardless of war era (Magruder \& Yeager, 2009).

Combat Exposure. Combat exposure is a significant predictor of PTSD. Among WWII and Korean War veterans, those who were exposed to moderate or heavy combat were 13.3 times more likely to have PTSD symptoms 45 years later (Spiro et al., 1994). Combat exposure has been found to be a significant predictor of PTSD symptoms immediately after deployment, as well as 18 to 24 months later (Sharkansky et al., 2000).

Research with OEF-OIF soldiers has had the additional advantage of occurring during wartime. Such studies have further helped uncover the relationship between combat exposure and PTSD. Exposure to combat among OEF-OIF veterans has been common. Between 50 to 60 percent of soldiers and marines reported having at least one combat experience, although the combat exposure of marines in both conflicts has been significantly higher (Mental Health Advisory Team, 2008). Research of OEF-OIF service members has shown that there is a linear relationship between the number of reported combat experiences and acute stress symptom severity (Mental Health Advisory Team, 2008), as well as PTSD symptom severity (Hoge et al., 2007). When units that were exposed to combat are surveyed, the prevalence of PTSD is higher than units that experienced less combat (Sundin et al., 2010).

Type of Combat. Type of combat is also related to PTSD symptoms. The most commonly reported combat experiences of OEF-OIF marines includes being shot at (97\%), being attacked or ambushed (95\%), seeing dead bodies or human remains (94\%), shooting or 
directing fire at the enemy (87\%), and seeing ill or injured women and children (83\%) (Hoge et al., 2007). However, increasing attention has also been paid to the influence of post-battle experiences, which are another source of PTSD. Post-battle experiences may include witnessing traumatic events, handling mutilated bodies, or witnessing the devastation that combat inflicts on the environment (Renshaw, 2011). People who have post-battle experiences rarely report feeling threatened by their experiences. Rather, post-battle experiences that result in PTSD most often do so due to feelings of shock and horror. Such symptoms can directly cause subsequent PTSD (Renshaw, 2011).

Sustaining Combat Wounds. Hoge et al. (2007) found that soldiers and marines who sustained a wound or injury were between two to three times more likely to have a diagnosis of PTSD. Furthermore, about one-third met at least one of the PTSD criteria. The manifestation of PTSD following combat wounds is often delayed. Among soldiers who were seriously wounded, the majority $(80 \%)$ did not meet criteria for PTSD one month after sustaining their injuries (Grieger et al., 2006). Grieger and colleagues found that the prevalence of PTSD among such soldiers increased with time and that the severity of their PTSD symptoms was highly related to the severity of their physical injuries.

In comparison to other conflicts, the type of wounds sustained by veterans of Iraq and Afghanistan are unique. Combat in World War II, Korea, and the Vietnam War was more likely to result in thoracic gunshot and shrapnel wounds, while service members in Iraq and Afghanistan have experienced and continue to experience significantly more head and neck wounds (Owens et al., 2008). Furthermore, Owens and colleagues (2008) found that 78 percent of the wounds suffered by OEF-OIF service members were sustained from explosions, often due to improvised explosive devices (IEDs). Blast-related injuries represent a higher percentage of 
total Iraq and Afghanistan combat injuries than all prior large-scale conflicts. Due to advancements in battlefield medicine, soldiers in Iraq and Afghanistan are significantly more likely to survive blast-related injuries - even when compared to survival rates during the Persian Gulf War. In Iraq and Afghanistan, a service member who sustains a blast-related injury can be transported to a modern medical facility within hours. While their physical wounds may be healed, soldiers who survive blast-related injuries are likely to have psychological wounds as well.

Blast related injuries are a significant cause of traumatic brain injuries (TBI). Much recent research has investigated the relationship between TBI and PTSD. One study found that the PTSD symptom severity of solders with blast-related TBI was not significantly higher than individuals who had non-blast related TBI (i.e. due to falls or motor vehicle accidents) (Kennedy, Leal, Lewis, Cullen, \& Amador, 2010). However, loss of consciousness was associated with significantly more severe PTSD. Also, veterans with blast-related TBI had significantly higher re-experiencing symptoms including more nightmares and flashbacks. Nearly 15 percent of soldiers reported sustaining an injury that involved loss of consciousness or an altered mental state (Hoge et al., 2008). Another study found that about 11 percent of soldiers met criteria for mild TBI (Mental Health Advisory Team, 2008). Hoge and et al. (2008) found that PTSD was strongly associated with mild TBI. About 44 percent of soldiers who reported loss of consciousness met criteria for PTSD compared with 27 percent who experienced altered mental status. In comparison, the prevalence of PTSD among soldiers who did not sustain a battle injury was around 9 percent.

Race \& Ethnicity. Race/ethnicity is one sociodemographic variable that has received increasing attention in the literature, in part due to the fact that racial/ethnic minority patients 
have traditionally been underrepresented in randomized clinical trials. However, studies investigating race/ethnicity as a predictor of PTSD have yielded conflicting findings.

Frueh, Gold, de Arellano, and Brady (1997) investigated racial differences on a number of symptom measures between Caucasian and African American Vietnam veterans. They did not find any significant racial differences on measures of anxiety, depression, PTSD, or personality, although some scales did trend toward statistical significance. There were also no between-group differences on clinician-rated Global Assessment of Functioning. However, a weakness of this study is an overly conservative statistical approach. Due to the large number of statistical analyses performed, the alpha level was set a priori to $p<.001$. Alternate statistical methods (e.g. using multivariate analysis of variance rather than multiple ANOVAs) and different procedures to adjust for Type I error may have resulted in different findings.

Frueh, Elhai, Monnier, Hamner, and Knapp (2004) also failed to find many significant racial differences in another sample of Caucasian and African American Vietnam veterans. There were no significant differences with respect to anxiety, depression, anger and hostility, psychotic symptoms, or level of dissociation. One additional study with a larger sample also failed to find significant racial differences between Caucasian and African American Vietnam veterans (Grubaugh et al., 2006).

In perhaps the largest study of Vietnam Veterans, the National Vietnam Veterans Readjustment Study (NVVRS) (Kulka et al., 1990), some significant racial differences did emerge. Kulka and colleagues (1990) found that the prevalence of PTSD was higher in African Americans (20.6\%) and Latinos (27.9\%) when compared to Caucasian veterans (13.7\%).

Additional studies have found that significant differences in PTSD do exist across race/ethnicity. Within the VA system, there is a higher prevalence of PTSD among minority 
veterans (Magruder \& Yeager, 2009). Schnurr, Lunney, and Sengupta (2004) found that Latino status was a significant premilitary factor associated with PTSD. Furthermore, minority status was significantly related to the maintenance of PTSD over time. An additional study found that the PTSD symptom severity of Latinos was significantly higher than for other populations (Marshall, Schell, \& Miles, 2009). Furthermore, Latinos with PTSD in this study had significantly higher reports of hyper-vigilance, flashbacks, intrusive thoughts, and emotional reactivity. A meta-analysis by Brewin et al. (2000) suggested that for military veterans, minority status is a significant risk factor for PTSD. In a more recent meta-analysis, Alcántara, Casement, and Lewis-Fernández (2013) found that Latinos were significantly more likely to meet criteria for PTSD following exposure to trauma. Latinos also were significantly more likely than nonLatinos (i.e. Caucasians and African Americans) to have higher and more severe PTSD symptoms.

Exposure to combat among OEF-OIF veterans has been extremely common. Between 50 to 60 percent of soldiers and marines reported having at least one combat experience, although the combat exposure of marines has been significantly higher (Mental Health Advisory Team, 2008).

There are multiple reasons why race and ethnicity may be related to PTSD. In prior conflicts such as the Vietnam War, African American and Hispanic service members were more likely than Caucasian service members to be assigned to positions and units that experienced combat. Race and ethnicity may also be a proxy for additional factors that lead to PTSD. Throughout the United States, racial and ethnic minority members of the population are more likely to live in areas with higher crime rates. There are additional societal reasons that may also explain the discrepancy. Members from racial and ethnic minority groups who are seeking out 
mental health treatment encounter more barriers than Caucasians. Reduced ability to pay for services, lack of access to services, and the stigma against receiving mental health treatment may cause people from racial/ethnic minority groups to delay seeking treatment until their symptoms are more severe than Caucasians. It may be the case that they do not present to treatment until the

Socioeconomic Status. The impact of socioeconomic status on PTSD is significantly less controversial. Low socioeconomic status (SES) is consistently implicated as a risk factor for PTSD. Not only does low SES increase the risk for developing PTSD following a traumatic event, but low educational attainment is also a factor in the persistence of PTSD (Schnurr et al., 2004). Alcántara et al. (2013) found that low income and low education were both associated with higher PTSD symptom severity. Brewin et al. (2000) found that low education was a significant risk factor. Much research has shown that low SES significantly increases the exposure of people to traumatic events. Less access to resources may also influence the methods by which people cope with trauma.

Additional Sociodemographic Risk Factors. A large number of additional sociodemographic risk factors have been implicated in the development of PTSD. Lack of social support, especially post-trauma, has consistently been found to be a significant PTSD risk factor (Brewin et al., 2000). Low social support at homecoming, as well as low current social support, are risk factors for veterans (Schnurr et al., 2004). The presence of relationship concerns during deployment is also an additional risk factor (Vogt et al., 2011). Vogt et al. (2011) suggested that relationship concerns may influence the risk of PTSD through their influence the perceived threat of combat experiences. Furthermore, Vogt et al. (2011) found that concerns about family disruptions during deployment are strong predictors of low social support post-deployment. 
Finally, in the general population, gender is also a risk factor for PTSD (Kessler et al, 1995).

Female status is significantly associated with an increased prevalence of PTSD.

\section{Psychotherapies for PTSD}

A large body of outcome research has attempted to determine the efficacy of psychotherapies for PTSD. A review of the meta-analyses of PTSD outcome research shows signs for encouragement as well as continued challenges in this area of treatment. The initial research on PTSD treatment outcomes sought to investigate the absolute efficacy of psychotherapy for PTSD. Absolute efficacy is defined as whether or not patients who participate in a therapy are better off than those with the same condition who receive no therapy. Additional studies have also sought to investigate the relative efficacy of the various treatments for PTSD. Such studies have determined which treatments for PTSD are best under which conditions and for which groups of people.

Meta-Analytic Findings. Shalev, Bonne, and Eth (1996) were among the first researchers to summarize this increasingly large body of research. In a review of over 80 studies they investigated the efficacy of biological, behavioral, cognitive, and psychodynamic therapy for the treatment of PTSD. The authors found that antidepressants were generally effective, although there were some conflicting results in the literature. Behavioral and cognitive interventions such as flooding, desensitization, and cognitive processing therapy (CPT) for PTSD were shown to be effective. However, following the interventions in many studies, many participants still had significant PTSD symptoms.

Sherman (1998) was one of the first researchers to use meta-analytic techniques to determine the overall efficacy of PTSD treatments. Meta-analysis allows for a quantitative comparison of effect sizes across dependent measures and across multiple studies. Sherman's 
(1998) meta-analysis included 11 studies of combat-related PTSD and 7 studies of traumarelated PTSD. These studies represented 690 total participants including over 350 combat veterans. Psychotherapies investigated included cognitive-behavioral therapy (CBT), exposure, and CPT. He found that the overall effect size (Cohen's $d$ ) immediately after treatment was .52 and .64 up six month follow-up. Sherman (1998) concluded that the magnitude of improvement was moderate and that PTSD treatments were effective at reducing PTSD symptoms, depression, and anxiety.

Bradley et al. (2005) examined 26 studies that looked at the efficacy of CBT, exposure, and eye movement desensitization and reprocessing (EMDR). They found that when all therapies were considered together, the pre- and post-treatment effect size (Cohen's D) was 1.44. When active treatments were compared with control treatments, termed "supportive counseling" by Bradley and colleagues, the effect size was smaller (Cohen's $D=0.83$ ). Thus, the absolute efficacy of PTSD treatments was supported and the overall effect size of treatment is large. Due to the relatively low number of studies included, Bradley and colleagues did not examine the relative efficacy of the different PTSD treatments. However, they did have some notable findings, which were all consistent with prior literature. Of perhaps most concern to veterans is that participants whose traumatic events occurred during combat experienced smaller treatment gains when compared with sexual/physical assault survivors as well as those who experienced mixed/other trauma. Furthermore, studies with more exclusionary criteria and studies where fewer patients complete the treatment have higher effect sizes.

Benish, Imel, and Wampold (2008) criticized the practice of comparing active psychotherapies with treatments that are not intended to be therapeutic, a limitation of many of the prior meta-analyses studies (e.g. Bradley et al. 2005). As a result, some authors concluded 
that some PTSD treatments were most effective while others argued there were no significant differences between treatments. Benish et al.'s (2008) meta-analysis included studies with comparison groups that met criteria described by (Wampold, Mondin, Moody, Stich, \& et al., 1997). Thus, only those studies with comparison groups that provided psychotherapies intended to be therapeutic were included. There were 15 studies that met the criteria with 958 total participants. The authors found there were no significant differences between any of the treatments investigated (e.g. CBT, CPT, PE, EMDR, \& stress inoculation). However, a main limitation of this meta-analysis is the relatively small number of studies that met criteria for inclusion.

Thus, the overall findings of the research on PTSD treatments overwhelmingly support absolute efficacy of many PTSD treatments. Furthermore, while there are many effective treatments for PTSD, some patient populations, such as combat veterans, seem to experience fewer benefits from participating in treatment. Given the large number of veterans in the VA system and the large prevalence of PTSD, the VA has prioritized the dissemination of empirically supported treatments (EST) for PTSD. Two of the most widely promoted treatments for PTSD are CPT and PE.

Cognitive Processing Therapy. Cognitive processing therapy (CPT) was originally developed by Resick and Schnicke (1992) for rape survivors. However, it was soon adapted for combat survivors. Its theoretical basis stems from social cognitive theory, as well as Foa and Kozak's (1986) Emotional Processing Theory. In addition to conceptualizing PTSD as the result of a maladaptive fear network, CPT also focuses on intense affective responses, such as guilt and anger. According to this view, there are two kinds of emotions. Primary emotions stem directly from the trauma, while natural emotions are the result of the person's interpretations of the 
event. For example, a person may experience a fight-or-flight response to a traumatic memory (primary emotion) and shame related to his or her inability to act in that moment (manufactured emotion). Social cognitive theory focuses on the content of cognitions and how people reconcile new information with existing schemas. People may assimilate information by altering it to fit their preexisting beliefs. People may also accommodate their beliefs to fit with new incoming information. Finally, people may over-accommodate by altering their beliefs in an extreme way in order to maintain a sense of safety or self-control. CPT focuses on stuck points, aspects of a trauma that result in over-accommodation and very strong primary and secondary emotions. Once activated, the affective elements associated with a trauma may be altered so that they are more adaptive. In addition to focusing on the cognitive components associated with the trauma, patients undergo exposure to these traumatic memories via a written account. CPT is typically conducted in 12 to 13 weekly sessions that are 60 minutes in length. CPT is commonly implemented in both individual and group psychotherapy.

A large number of clinical trials have demonstrated CPT's absolute efficacy and shown that it is equivalent to many other active psychological treatments for PTSD. Resick, Nishith, Weaver, Astin, and Feuer (2002) directly compared the efficacy of CPT to PE and a waitlist control group. They found that both active treatments were superior to the waitlist control and that there were no significant differences between the two active treatments. Two clinical trials (Chard, 2005; Monson et al., 2006) confirmed that CPT was superior to waitlist control and also showed that the improvements were sustained up to one year post-treatment. Resick et al. (2008) conducted a dismantling study that compared the efficacy of traditional CPT, as well as two components of CPT: (1) written account only and (2) CPT-Cognitive. All treatments were effective. However, the cognitive-only component of CPT was as effective as the traditional CPT 
protocol, which indicates the written account may not be necessary for some patients to benefit from the treatment.

Additional studies have examined the effectiveness of CPT in settings that were less restrictive than is typical of randomized clinical trials. Galovski, Blain, Mott, Elwood, and Houle (2012) investigated the effectiveness of an altered CPT protocol in a community sample. The researchers altered termination criteria to be more flexible to patient needs. They found that modified CPT was still effective. Nixon (2012) compared the effectiveness of CPT and supportive counseling in a community sample that had experienced trauma. Results indicated no statistically significant differences between the two treatments, although patients in the CPT group had larger effect sizes on some outcome measures.

Multiple studies that evaluated the effectiveness of CPT have been carried out in VA settings with veterans (e.g., Alvarez et al., 2011; Chard, Schumm, Owens, \& Cottingham, 2010; Chard et al., 2012; Forbes et al., 2012). All found that CPT was effective at reducing PTSD symptoms in veterans. Chard et al. (2010) found that OEF-OIF veterans experienced significantly greater improvements from CPT and Vietnam veterans. However, limitations of these studies are that they either had pre-post designs (Alvarez et al., 2011; Chard et al., 2010; Chard et al., 2012) or contained a small number of participants (Forbes et al., 2012; number of participants in CPT $=29$ ). Although pre-treatment data for patients who eventually dropped out from treatment was available, post-treatment data for non-completers was unavailable. No studies to date have tracked the progress of treatment non-completers through the therapy sessions they did attend.

Prolonged Exposure. PE was originally developed by Foa, Rothbaum, Riggs, and Murdock (1991) for the treatment of rape victims. It was soon adapted for the treatment of 
combat veterans. Its theoretical underpinnings can be traced to classical and operant conditioning. PE is based on the idea that fear is acquired via classical conditioning by the association of stimuli that were present at the time of the trauma with a person's intense emotional response to that trauma. This association is maintained through operant conditioning, specifically negative reinforcement. Once the association has been made, individuals avoid unwanted stimuli (e.g. triggers, negative emotions), which maintains the anxiety response. Thus, by avoiding these stimuli, a person with PTSD never becomes habituated to the anxiety response and will continue to experience trauma symptoms.

PE is based on Foa and Kozak's (1986) Emotional Processing Theory (EPT). EPT states that people form fear structures to help them avoid or escape danger. Fear structures are a person's representation of feared stimuli, their responses to those stimuli, and the meaning they give to those stimuli and responses. Fear structures are activated by input that matches information stored in the fear structure. EPT states that extinction of a pathological fear structure occurs when we activate that fear structure and incorporate new information into that fear structure. This information involves the removal of erroneous associations and the formation of more accurate attributions. While the old, pathological connections are thought to still exist, these new and more adaptive connections are thought to compete with the old connections. The primary mechanism of change in PE is emotional processing, which occurs through in vivo exposure to traumatic memories and the processing of those memories. PE is typically conducted in 8 to 15 sessions that are between 60 and 90 minutes in length (Foa, 2011). In practice, PE often focuses on the most devastating traumatic memory.

PE has been one of the most heavily studied ESTs for PTSD. In a recent meta-analysis, (Powers, Halpern, Ferenschak, Gillihan, \& Foa, 2010) summarized the results of 13 studies that 
represented 658 participants. There was strong evidence for PE's absolute efficacy. When compared with a waitlist control group, PE had an effect size (Hedge's $g$ ) of 1.51. PE's treatment effects were sustained through follow up. PE was found to be equivalent to CPT, EMDR, and stress inoculation training. All active treatments were found to be equally effective (Hedge's $g=$ -0.07 .

Studies of the effectiveness of PE in settings more reflective of clinical practice have largely matched the results from more controlled studies. Multiple studies have been conducted with combat veterans in the VA system (Eftekhari et al., 2013; Goodson et al., 2013; Rauch et al., 2009; Tuerk et al., 2011; Yoder et al., 2012). All of the effectiveness studies carried out with vetrans have shown that PE is an effective treatment for PTSD. Eftekhari et al.'s (2013) study is noteworthy because it evaluated the national implementation of PE throughout the VA system and included a sample of over 1900 veterans. An important finding from Yoder et al.'s (2012) study was that Persian Gulf War veterans responded significantly less well to treatment than both Vietnam and OEF-OIF veterans. However, the above effectiveness studies tend to have similar limitations. Studies either tend to have a relatively small sample size ( $n=115$ or lower) (e.g., Goodson et al.,2013; Rauch et al., 2009; Tuerk et al., 2011) and/or a pre-post design that does not enable the tracking of treatment non-completers over time (e.g., Eftekhari et al., 2013).

Direct Comparisons of CPT and PE. Direct comparisons of CPT and PE have been limited. A literature review revealed only two studies that directly compared the relative efficacy of these psychotherapies. Resick et al. (2002) randomly assigned a sample of 171 female rape victims to receive either $\mathrm{CPT}$, PE, or minimal attention. They found that both treatments were highly effective and superior to the minimal attention condition. Regarding relative efficacy, Resick et al. (2002) also found that treatment outcomes between CPT and PE were largely the 
same. Resick and colleague's results did suggest that CPT was superior at reducing guilt-related cognitions. Jeffreys et al. (2014) compared the effectiveness of CPT and PE in a sample of veterans. Jeffreys and colleagues found that both treatments significantly reduced self-reported PTSD symptoms, but that PE $(d=2.0)$ was superior to CPT $(d=0.96)$.

Promotion of CPT and PE in the VA System. In light of the empirical support in the literature for $\mathrm{CPT}$ and $\mathrm{PE}$, the VA implemented a widespread training program to teach mental health providers CPT and PE in 2007(U.S. Medicine, 2009). The training program involves experiential and didactic training for mental health providers and delivers individual consultation for therapists on at least two cases so that they can gain further experience delivering the treatments. As of 2009, nearly 3,000 staff had been trained in at least one of the two treatments (U.S. Medicine, 2009).

\section{Factors Associated With Patient Outcomes}

Time and Session Attendance of Treatment. For individuals receiving CPT or PE, their PTSD symptoms decrease over time as individuals attend more therapy. The meta-analytic findings of PTSD treatments confirm that while there is room for improvement, people who participate in treatment do get better. Therefore, when evaluating the PTSD symptoms of individuals who are receiving mental health treatment, time and session attendance are two of the most important predictors of PTSD.

Initial PTSD Symptom Severity. Recent research has shown that the severity of PTSD symptoms can also influence how much patients benefit from treatment. Jeffreys et al. (2014) suggested that patients who begin treatment with more severe symptoms experience greater gains in therapy than patients who begin treatment with lower symptoms. Schumm, Walter, and Chard (2013) showed that patients with different initial severities of PTSD symptoms report different 
trajectories of improvement. It is possible that the different levels of improvement may be real effects. However, measurement issues such as ceiling effects may also explain why patients who begin treatment with fewer PTSD symptoms report fewer gains as a result of therapy.

Race/Ethnicity. Despite the high amount of research into the efficacy and effectiveness of PE and CPT, there has been limited research on the relationship of race/ethnicity with therapy outcomes. Findings regarding the potential influence of race/ethnicity on treatment outcomes have been conflicted. Some researchers have found members of racial/ethnic minority groups have less favorable outcomes while others have found no relationship between race/ethnicity and outcomes in therapy.

The American Psychological Association (2003) defines race as "the category to which others assign individuals on the basis of physical characteristics, such as skin color or hair type, and the generalizations and stereotypes made as a result." Ethnicity is "acceptance of the group mores and practices of one's culture of origin and the concomitant sense of belonging...individuals may have multiple ethnic identities that operate with different salience at different times."

Helms (1984) proposed a theoretical model that described the various stages of racial awareness for African Americans and European Americans and hypothesized how the interaction of race and racial awareness might influence psychotherapy outcomes. She described four potential kinds of therapeutic relationships. Parallel relationships occur when the counselor and client share the same attitudes about race. Crossed relationships occur when the counselor and client are in opposite stages of racial awareness. Progressive relationships occur when the counselor's racial awareness is at least one stage more advanced than the client's. Finally, regressive relationships occur when the client's stage of development is at least one stage more 
advanced than the counselor's. Helms (1984) hypothesized that in counselor-client dyads with mixed races some interactions (e.g., parallel and progressive) would be more likely to result in long-lasting therapeutic relationships with more beneficial outcomes. Meanwhile, other interactions (e.g., crossed and regressive) would be more likely to lead to premature termination and the absence of positive changes for the client.

Carkhuff and Pierce (1967) investigated the effect of counselor socioeconomic status (SES) and race on the amount of self-exploration engaged in by patients. In one of the first quasiexperiments to investigate the influence of race and SES on the counseling process, they found that both counselor race and counselor SES had an effect on the amount of client selfexploration. Those clients of minority status who were from low SES backgrounds had the lowest amount of self-exploration.

Wierzbicki and Pekarik (1993) conducted a meta-analysis of 125 studies of psychotherapy dropout. They found that membership in a racial/ethnic minority group, low income, and low education were all positively associated with higher rates of dropout from therapy. Walling et al. (2012) investigated differences between racial/ethnic majority and minority clients in the trajectory of the working alliance and linked that to therapy outcomes. Majority clients' working alliance trajectories tended to show growth over time, which was related to more beneficial outcomes. Minority client ratings of the working alliance were more likely to be stagnant, which was related to worse outcomes six months after therapy. Thus, client race is a significant variable that can influence both the duration and process of therapy.

Of the studies that investigated the efficacy or effectiveness of CPT and/or PE, only three studies directly tested the influence of race/ethnicity on treatment outcomes. Tuerk et al. (2011) found that race/ethnicity was related to significantly higher self-reported PTSD symptoms. Tuerk 
et al. (2011) did not find that race/ethnicity predicted any clinical or statistical differences after the patients completed PE. Consistent with the prior literature, Resick et al. (2008) found that minority status was significantly associated with a lower rate of treatment completion. However, Alvarez et al. (2011) did not find any significant treatment effects of CPT due to race/ethnicity. Unfortunately, studies that test for treatment effects associated with race/ethnicity are the exception, rather than the rule. When race/ethnicity is tested, it is often collapsed all minority racial groups into one category that is very broad. Then, the therapy outcomes for majority and minority clients are directly compared despite the fact that the within-group variance for minority clients is quite high. Ten studies reported the race/ethnicity of their participants, but did not test for the effect of race/ethnicity on treatment outcomes. The most common reason for this omission was low representation of racial/ethnic minority patients in these studies or low overall sample sizes, both common limitations in clinical research (Chambless \& Hollon, 1998).

Studies of combat veterans receiving treatment for PTSD have shown that individuals who participate fully in treatment have equivalent treatment outcomes, regardless of race and ethnicity (Rosenheck \& Fontana, 1996; Rosenheck, Fontana, \& Cottrol, 1995). However, the research on the utilization of mental health treatment by veterans has revealed some important racial and ethnic differences. African American veterans receiving PTSD treatment attended fewer sessions (Rosenheck, Fontana, \& Cotrol, 1995; Elhai, Reeves, \& Frueh, 2004; Lester, Resick, Young-Xu, \& Artz; 2010).

Therapist Effects. In most psychotherapy outcome research, therapist effects are treated as confounding variables - if they are mentioned at all. Most researchers investigating the efficacy or effectiveness of CPT and PE have not taken into account the potential influence of therapist effects. Wampold (2001) showed that ignoring nested data common in psychotherapy 
outcome research can lead to inflated Type I error and an overestimation of treatment effects. Observations within nested data are linked, so ignoring nested data violates an assumption that both tests make regarding the independence of observations, which can result in biased statistical tests (Peugh, 2010). The present study will account for therapist effects in a post-hoc analysis to help control for this potential source of bias.

\section{Limitations of CPT and PE}

Both CPT and PE are not without their limitations. Therapists who provide CPT or PE must engage in a balancing act that communicates the rationale for these treatments and the importance of confronting trauma-related thoughts and memories despite the tendency of people with PTSD to engage in avoidance. Many patients with PTSD have a difficult time confronting avoidance, so dropout from these treatments is a common problem. Furthermore, the severity of PTSD experienced by veterans results in many patients continuing to meet diagnostic criteria for PTSD after treatment. Many others will continue to experience symptoms that are clinically significant, though below the diagnostic threshold for PTSD. This section reviews issues related to dropout from treatment and common barriers that prevent veterans from receiving treatment.

Dropout. Estimates of dropout from CPT and PE vary across studies, due in part to differences in how dropout is defined. Dropout has been defined as failure to complete a full psychotherapy protocol (e.g., Monson et al., 2006), attending less than two-thirds of the required number of sessions (Jeffreys et al., 2014), and failure to return to a previously scheduled appointment (Chard et al., 2010). Acomparison of dropout rates between treatments should also take into account the 8 -session requirement for PE and 12-session requirement for CPT. When dropout is defined as failure to complete the full therapy protocol, estimates of dropout for CPT among veterans is $20 \%$ (Monson et al., 2006). Under the same definition, dropout rates for PE 
are about 28\% (Eftekhari et al., 2013). When dropout is defined as failure to complete two-thirds of the protocol, Jeffreys et al. (2014) found that dropout from PE (44\%) was greater than for CPT (32\%). Furthermore, there is evidence that dropout is related to additional factors such as age. Chard et al. (2010) found that $35 \%$ of OEF/OIF/OND and $26 \%$ of Vietnam veterans dropped out from CPT. In Chard et al.'s study (2010) dropout was defined as failure to return to any session that had been scheduled. Jeffreys et al. (2014) also found that younger age was associated with significantly higher dropout from therapy.

Barriers to Treatment. The stigma associated with mental illness - especially within the military—has prevented many individuals from seeking treatment (Stecker, Shiner, Watts, Jones, \& Conner, 2013). Within a military sample, Hoge et al. (2007) found that less than half of those diagnosed with a mental health problem were interested in receiving help. Common concerns raised by members of the military included perceptions that they would be seen as weak, be treated differently by commanders and fellow soldiers, and that seeking treatment would harm their future career.

Negative attitudes regarding mental health disorders and mental health treatment often persist after a person has discharged from the military. In a study of OEF-OIF veterans with PTSD who decided not to receive treatment, many veterans cited stigma as a main barrier to treatment (Stecker et al., 2013). However, Stecker et al. (2013) found that concerns regarding treatment and emotional readiness for treatment were even more common. Concerns about treatment often involved the concern that PTSD treatment would require medication. Veterans often reported wanting individual rather than group treatment and often held the belief that their providers would not understand their problems. Concerns regarding emotional readiness for treatment were expressed by about one third of the veterans who did not seek PTSD treatment. 
Veterans often believed that despite their suffering from PTSD, they did not need treatment or that it would be too difficult to talk about their PTSD. Logistical concerns such as not having enough time for treatment or living too far away from a treatment center were also issues that veterans commonly raised. Logistical concerns continue to disproportionally impact veterans who live in rural areas (APA, 2013), although the recent increase in telemedicine has helped to improve the situation.

\section{Summary and Limitations of the Literature}

The literature on PTSD and PTSD treatments suggests a number of important findings. PTSD is a very common disorder among veterans of the armed forces. PTSD has been associated with a number of harmful health outcomes. The cost that PTSD incurs on individuals and to society is very high. There are a number of important predictors of PTSD including peritraumatic dissociation, social support, level of combat exposure, and cognitive and emotional reactions to trauma. Individual characteristics of veterans such as age is also a predictor of PTSD. Although the influence of race and ethnicity is subject to debate, it does appear that minority veterans are significantly less likely to utilize mental health services from the VA system. Such clients appear to be more likely to drop out from PTSD treatments.

Encouragingly, it does appear that we have effective treatments for PTSD. CPT and PE are two of the most common treatments in the VA system and are among the most effective PTSD treatments. However, research on these treatments has some important limitations. With the exception of Chard et al. (2012), Eftekhari et al. (2013), and Jeffreys et al., (2014), the majority of studies on CPT and PE with veterans have involved fewer than 120 participants. The representativeness of racial/ethnic minority groups compared to the general population of veterans in this body of literature tends to be lacking. Although effectiveness research of CPT 
and PE is growing, additional research could help to verify the effectiveness of CPT and PE outside of a controlled clinical environment.

Most of the outcome research to date has involved a pre-post design and a relatively few number of studies have modeled the trajectory of PTSD symptoms over time. Although one study to date (Jeffreys et al., 2014) has used a retrospective chart review to compare the effectiveness of CPT and PE in the VA, no studies to date have compared the trajectories of PTSD symptom reduction using multilevel modeling. Furthermore, no studies to date have investigated the average trajectory of patients who drop out from treatment to rule out potential iatrogenic effects of these psychotherapies.

Relatively few researchers of trauma-focused therapy have investigated the relationship between race/ethnicity and treatment outcomes. When researchers have investigated race/ethnicity, they often collapse minority groups together. This approach is common among those researching ESTs, although the importance of race/ethnicity has become more appreciated in recent years. However, racial/ethnic minority patients have historically been underrepresented in EST research (APA, 2003). Many have argued we must make our research of ESTs more inclusive of all patients, especially those who are from racial/ethnic minority groups (see for example, Sue \& Zane, 2006).

The present study compared the effectiveness of CPT and PE delivered in individual therapy for the treatment of PTSD. The study used a retrospective, quasi-experimental design. Assignment into treatment groups wass nonrandom because it was influenced by the decisions of mental health providers and the veterans receiving the treatment. Given the large amount of evidence supporting the absolute efficacy of PE and CPT, no placebo group or waitlist control was included in the study. Participants included veterans who received CPT or PE in VA settings 
in ten states across the country. Data were obtained from the VA Informatics and Computing Infrastructure (VINCI). The primary outcome measure was self-reported PTSD symptoms on the Posttraumatic Stress Disorder Checklist (PCL).

The current study used multilevel modeling to determine the absolute and relative effectiveness of both treatments. A two-level, hierarchical model was tested, which included repeated observations over time (level 1) nested within patients (level 2). The study also tested multiple patient-level factors that, based on the prior literature, have the potential to be significantly related the effectiveness of both treatments. The present study tested the relationship between the following patient-level factors and the changes in self-reported PTSD symptoms over time (i.e., the trajectory): completion status, initial PTSD symptom severity, race/ethnicity, and age. A post-hoc analysis was used to determine the size of therapist effects in the current study's sample to evaluate this potential source of bias in estimating the effects of the two treatments.

\section{Research Questions \& Hypotheses}

The following research questions and hypotheses guided this study.

Research Question 1. What is the absolute and relative effectiveness of PE and CPT?

- Hypothesis 1: Both treatments will result in a significant reduction in PTSD symptoms over time.

- Hypothesis 2: Both treatments will be equally effective. That is, there will be no difference between treatment groups with respect to the rate of symptom improvement, irrespective of pre-treatment PTSD symptom severity.

Research Question 2. Which patient-level variables are significantly related to the average growth trajectory of each treatment? 
- Hypothesis 3: The following patient-level variables are predicted to be significantly correlated with the growth trajectory of PTSD symptoms: session attendance, initial PTSD symptom severity, and age at beginning of treatment.

- Hypothesis 4: The trajectory of PTSD symptoms for Caucasian, African American, and Hispanic veterans is predicted to be identical, irrespective of pre-treatment differences between racial/ethnic groups with respect to PTSD symptom severity. 


\section{Chapter III}

\section{Methods}

This study was designed to determine the absolute and relative effectiveness of CPT and PE within VA settings across the country. An additional goal of the study was to determine the relationship between patient-level variables such as completion status, initial PTSD symptom severity, age, and minority status with the trajectory of PTSD symptom reduction. This chapter describes the study's participant selection and data cleanup procedures, independent variables, measures, procedure, data analysis, research questions, and hypotheses.

\section{Procedure}

The study used retrospective data and required the use of protected health information (PHI) such as social security numbers and dates of birth. All data, including personallyidentifiable records, was stored on the secure VINCI drive to which only the principal and coinvestigator (Dr. Mary Oehert) had access. The data were accessed via a secure VINCI server. Chart reviews were conducted using the Compensation and Pension Records Interchange. The investigator performed all data clean up and statistical analyses on the secure VINCI workspace using SPSS and Microsoft Excel. Raw data from this study were not removed from the VAprotected environment. The researcher did access the data via remote access.

No raw data, including PHI, were removed from the secure research drive or made available to individuals not involved in the study. The results of aggregated statistical tests were transferred from the VINCI workspace to facilitate the reporting of overall study results. To protect confidentiality, all individually identifiable information was be transformed or deleted as soon as it was no longer needed. 
Patient Selection. Patients who completed the Posttraumatic Stress Disorder Checklist (PCL) three or more times within a six month period from January 1st, 2006 to July 31st, 2013 were randomly selected for a chart review. Both the PE and CPT protocols recommend regular administration of the PCL on a weekly basis during treatment. However, these measures are administered quite frequently in the VA system in contexts other than psychotherapy. The threshold of three or more completed PCLs was chosen in an attempt to filter out individuals who did not receive mental health treatment while increasing the likelihood of retaining people who did not complete the treatments.

The data selection procedure targeted specific Veterans Integrated Service Networks known to be high in diversity according to the 2010 U.S. Census. VA Medical Centers and Community-Based Outpatient Clinics in Alabama, Arizona, California, Georgia, Illinois, Kansas, Missouri, New York, Texas, and Wisconsin were included in this study. Since all data were retrospective, decisions regarding the assignment of participants to treatment groups were based on the clinical judgment of mental health providers, treatment teams, as well as the consent of the individual patients. The main inclusion criterion was whether patients had received CPT or PE in individual therapy.

In keeping with the principles of effectiveness research, exclusionary criteria for the study were limited. The goal was to reflect, as accurately as possible, the overall population of veterans in VA settings that have received PE and CPT in individual therapy. However, data from clinical work in real world settings also tends to be highly unbalanced. The number of measurements each patient received and the interval between measurements was highly varied. Data that are structured in this way may bias parameter estimates in multilevel models. Additional data preparation steps were taken to limit this source of bias. 
Two thousand thirty patients' charts were reviewed. One thousand thirty patients were identified as having received either CPT or PE. Eight hundred forty patients received one of those treatments in individual therapy. Ninety patients were excluded from statistical analyses for the following reasons: (1) the patient's initial measurement did not take place until after the third session ( $n=20)$, (2) the treatment consisted of a combination of individual and group psychotherapy $(n=23),(3)$ there was a gap of six or more sessions between measurements $(n=$ $29)$, or (4) patients who received more than thirty weeks of individual therapy $(n=18)$. PCL scores for thirty eight patients who received more than fourteen sessions of psychotherapy were also removed from the dataset. These scores were removed because the trajectory of symptoms reported by these patients were not reflective of the rest of the group and held the potential to bias the multilevel model. However, the data for the first fourteen sessions these patients completed was included in the statistical models. Twenty nine of these patients received PE while eight had received CPT. Thus, the final sample was seven hundred fifty patients.

Power analysis. Given the conflicting findings in research on the effect size of race/ethnicity on treatment outcomes, it was difficult to estimate the effect size of race/ethnicity on treatment outcomes. For the present study, it was assumed that the targeted effect size for race/ethnicity would be small $(d=0.15)$. In a power analysis conducted using G-Power version 3.1.3 (Faul, Erdfelder, Lang, \& Buchner, 2007), a hypothetical study with six treatment groups and six measurements over time would have a $95 \%$ chance of finding a true effect size of .15 if the sample contains 300 participants. A low effect size of .15 was intentionally chosen. However, this power analysis did not take into account that the study would use multilevel modeling. To increase power, a minimum sample size of 600 was used. The selection of a larger number of participants will result in more accurate parameter estimates for the multilevel model. 


\section{Participants}

Demographics. Demographic information and descriptive statistics for the final sample are located below in Table 1.83 percent $(n=625)$ of the patients were male. The mean age $(S D)$ was 46.0 (15.6). However, there was a bimodal distribution of age due to two cohorts of veterans: Vietnam veterans $($ mode $=65.2)$ and $\mathrm{OEF} / \mathrm{OIF} / \mathrm{OND}$ veterans $($ mode $=32.6)$. The sample was 58.7 percent White $(n=440), 16.3$ percent African American $(n=122)$, and 17.0 percent Hispanic $(n=126)$. The most common mental health diagnoses were PTSD $(92.9 \%)$, a depressive disorder (61.7\%), a substance use disorder (29.0\%), and an anxiety disorder (20.7\%). Nearly three out of four patients in the sample had at least two mental health diagnoses. The mean $(S D)$ number of mental health diagnoses was $2.5(1.3)$. 87.1 percent of the veterans had a service connection $(n=653)$. The mean $(S D)$ percentage of service connection was $66.2(24.8)$ percent for those veterans who had service connections.

The type of therapy provided to the patients was evenly split between CPT (50.1\%) and PE (49.9\%). The mean (SD) number of sessions attended for CPT was 8.5 (3.8). The mean $(S D)$ number of sessions attended for PE was 8.0 (3.5). 47.6 percent $(n=357)$ of patients were defined as treatment completers (12 or more sessions for CPT; 8 or more sessions for PE). PE had more treatment completers $(55.9 \%)$ than CPT $(39.4 \%)$ due to the different requirements for treatment completion between the two treatments. One hundred sixty two therapists provided psychotherapy to the patients in this study. Providers most often consisted of psychologists (44.4\%), social workers (19.1\%), and psychology interns (13.6\%). For patients who received CPT, 59.8 percent were White, $12.5 \%$ were African American, and 18.9 percent were Hispanic. For patients who received PE, 57.5\% were White, 20.1 percent were African American, and 14.7 percent were Hispanic. 
Table 1. Demographics of sample and descriptive statistics. $(N=750)$

\begin{tabular}{|c|c|c|c|c|}
\hline Variable & $N$ & $\%$ & $M$ & $S D$ \\
\hline \multicolumn{5}{|l|}{ Sex } \\
\hline Men & 625 & 83.3 & & \\
\hline Women & 125 & 16.7 & & \\
\hline Age (years) & & & 46.01 & 15.55 \\
\hline \multicolumn{5}{|l|}{ Race/ethnicity } \\
\hline White & 440 & 58.7 & & \\
\hline African American & 122 & 16.3 & & \\
\hline Hispanic & 126 & 16.8 & & \\
\hline Asian, Pacific Islander, or Alaska Native & 24 & 3.2 & & \\
\hline American Indian or Alaska Native & 7 & 0.9 & & \\
\hline Unknown or Declined & 31 & 4.1 & & \\
\hline \multicolumn{5}{|l|}{ Service connection status } \\
\hline Connected (Mean Service Connection) & 653 & 87.1 & & \\
\hline Not Service Connected & 97 & 12.9 & & \\
\hline Service Connection Percentage & & & 66.17 & 24.84 \\
\hline \multicolumn{5}{|l|}{ Service era } \\
\hline WWII-post-Vietnam & 291 & 38.8 & & \\
\hline Persian Gulf-Present & 459 & 61.2 & & \\
\hline Initial Total PCL Score & & & 61.73 & 12.15 \\
\hline \multicolumn{5}{|l|}{ Type of Therapy Provided } \\
\hline Cognitive Processing Therapy & 376 & 50.1 & & \\
\hline Prolonged Exposure & 374 & 49.9 & & \\
\hline \multicolumn{5}{|l|}{ Number of Sessions } \\
\hline Cognitive Processing Therapy & & & 8.54 & 3.78 \\
\hline Prolonged Exposure & & & 7.98 & 3.52 \\
\hline \multicolumn{5}{|l|}{ Completed Full Therapy Protocol? } \\
\hline Yes & 357 & 47.6 & & \\
\hline No & 393 & 52.4 & & \\
\hline \multicolumn{5}{|l|}{ Provider Type } \\
\hline Psychologist & 72 & 44.4 & & \\
\hline Psychology Resident & 12 & 7.4 & & \\
\hline Psychology Intern & 22 & 13.6 & & \\
\hline Psychology Practicum Student & 12 & 7.4 & & \\
\hline Social Worker & 31 & 19.1 & & \\
\hline Social Work Practicum Student & 6 & 3.7 & & \\
\hline Licensed Master's-level Counselor & 1 & 0.6 & & \\
\hline Nurse & 4 & 2.5 & & \\
\hline Psychiatrist & 2 & 1.2 & & \\
\hline Total Number of Providers & 162 & & & \\
\hline Number of Patients per Provider & & & 4.60 & 6.61 \\
\hline
\end{tabular}




\begin{tabular}{|c|c|c|c|c|}
\hline \multicolumn{5}{|l|}{ Treatment Location (number of sites) } \\
\hline Arizona $(N=1)$ & 306 & \multicolumn{3}{|l|}{40.8} \\
\hline California $(N=3)$ & 178 & \multicolumn{3}{|l|}{23.7} \\
\hline Georgia $(N=1)$ & 67 & \multicolumn{3}{|l|}{8.9} \\
\hline Missouri/Kansas $(N=3)$ & 54 & \multicolumn{3}{|l|}{7.2} \\
\hline Wisconsin $(N=1)$ & 42 & \multicolumn{3}{|l|}{5.6} \\
\hline Illinois $(N=1)$ & 39 & \multicolumn{3}{|l|}{5.2} \\
\hline Texas $(N=1)$ & 29 & \multicolumn{3}{|l|}{3.9} \\
\hline New York $(N=1)$ & 23 & \multicolumn{3}{|l|}{3.1} \\
\hline Alabama $(N=1)$ & 12 & \multicolumn{3}{|l|}{1.6} \\
\hline \multirow[b]{2}{*}{ Variable } & \multicolumn{2}{|c|}{ CPT } & \multicolumn{2}{|c|}{ PE } \\
\hline & $N$ & $\%$ & $N$ & $\%$ \\
\hline \multicolumn{5}{|l|}{ Race/ethnicity } \\
\hline White & 225 & 30.0 & 215 & 28.7 \\
\hline African American & 47 & 6.3 & 75 & 10.0 \\
\hline Hispanic & 71 & 9.5 & 55 & 7.3 \\
\hline Asian, Pacific Islander, or Alaska Native & 12 & 1.6 & 12 & 1.6 \\
\hline American Indian or Alaska Native & 3 & 0.4 & 4 & 0.5 \\
\hline Unknown or Declined & 18 & 2.4 & 13 & 1.7 \\
\hline \multicolumn{5}{|l|}{ Completed Full Therapy Protocol? } \\
\hline Yes & 148 & 39.4 & 209 & 55.9 \\
\hline No & 228 & 60.6 & 165 & 44.1 \\
\hline
\end{tabular}

\section{Independent Variables}

Time. Time was operationalized as number of weeks and was included as a level 1 predictor. Time was centered so that Time $=0$ was session 1 of the treatment received by each patient.

Sessions Attendance. Session attendance was operationalized as a binary variable. This variable was included in analyses as a level 2 predictor. Patients who completed a full protocol of CPT ( $n$ of sessions $\geq 12$ ) and PE ( $n$ of sessions $\geq 8$ ) were considered treatment completers (completer $=1$ ). Patients who received fewer than the above number of sessions in each treatment were considered treatment non-completers (non-completer $=0$ ).

Initial PTSD symptom severity. Initial PTSD symptom severity was defined as the first PCL measurement available for each patient. It was included in the analyses as a level 2 
predictor. Session 1 PCL scores were available for 83.5 percent $(n=626)$ of the participants. Session 2 PCL scores (13.2 percent of sample; $n=99)$ and session 3 PCL scores (3.3 percent of sample; $n=25$ ) were used when session 1 PCL scores were unavailable. Initial PCL scores were included in all models as a mean-centered variable.

Age. Age was operationalized as the number of years old the patient was at the beginning of treatment. It was included as a mean-centered variable.

Treatment. The treatment modality for all patients was individual psychotherapy. Treatment was operationalized as participation in CPT $($ Treatment $=1)$ or PE $($ Treatment $=0)$ and was included as a level-2 predictor. For patients who received multiple treatments, the data for the first psychotherapy they participated in was included. All subsequent data for those patients was removed from the analysis.

Race/Ethnicity. The race/ethnicity of each client was included as an additional independent variable. Due to their prevalence within the VA system, three racial/ethnic groups were included in the study: Caucasians, African Americans, and Hispanics. These variables were reported by veterans upon their entry into the VA system. African American and Hispanic status was included as two separate binary variables in the model. African American status was coded as $1=$ yes, $0=$ no. Hispanic status was coded as $1=$ yes, $0=$ no. Both variables were included as level 2 predictors.

\section{Dependent Variable}

The primary dependent variable for this study was self-reported PTSD symptoms on the PCL (Weathers, Litz, Herman, Juska, \& Keane, 1993). The PCL has 17 items based on the DSM$I V$-TR criteria for PTSD. Respondents rate the frequency of each PTSD symptom on a 5-point Likert scale ( 1 = not at all; $2=$ a little bit; $3=$ moderately; $4=$ quite a bit; and $5=$ extremely $)$. 
The total score was computed by summing all of the individual items. Lower total scores indicate fewer and less severe PTSD symptoms while higher scores indicate more frequent and more severe PTSD symptoms. Subscales on the PCL include re-experiencing, avoidance/numbing, and hyperarousal. A confirmatory factor analysis with the current study's data was not possible because individual item responses were not collected. It should be noted that because the PCL is a measure of PTSD symptoms, decreases on the PCL over time represent therapeutic gains. Weathers et al. (1993) recommended a cut-score of 50 for the diagnosis of PTSD. The National Center for PTSD (2014) recommends a decline of 5 points as a minimum threshold for determining whether a person has responded to treatment. A 10 point decline is the minimum threshold for clinically meaningful change. Internal consistency for the PCLC is very high ( $\alpha$ $=.97)$. Test-retest reliability over a one week time period is also very high $(r=.96)$. Since its adoption in the 1990s, the PCL has become one of the most commonly used self-report measures of PTSD symptoms.

\section{Ethical Issues}

Ethical issues need to be managed in all forms of research, including the present study. Primary ethical issues that need to be managed in the present study include client confidentiality and autonomy. It is hoped that the security measures outlined above minimized risks to participants by ensuring that confidentiality is maintained. Specifically, the location of all data on a secure VINCI server and the removal of individual identifiers from study-related files as soon as they were no longer needed helped reduce the risk of any breaches of confidentiality.

The current study also received approval for an exemption of informed consent.

Obtaining informed consent from each participant would have been impractical for a number of reasons. The amount of time it would take to directly contact participants would have made the 
study unfeasible to carry out. Furthermore, directly contacting clients who have received mental health treatment could have resulted in further breaches of confidentiality since it would have identified them as former recipients of mental health treatment. All clients who enter the VA system grant consent for their data to be used in research. To protect the research participants in the current study, all procedures and ethical issues were approved by the internal review board of the VA Eastern Kansas Healthcare system. The study was also approved by the University of Kansas Human Subjects Committee of Lawrence.

Social security numbers were required in order to access the patients' charts. Once the chart review was completed, all patients were assigned a study-related identification number and their social security numbers were deleted from the study dataset. The actual dates that treatment was initiated and terminated were required for the data analysis. Having these dates enabled centering of the Time variable. All dates were transformed using Microsoft Excel. Then, time was centered at the beginning of treatment. All original dates were then deleted from the study dataset so that it would be impossible to trace the date back to the individual patients in the study.

Dates of birth were used to calculate the age of each patient during treatment. It was transformed in the same way as outlined above. Once transformed, the original date of birth was deleted from the study dataset. In order to account for therapist effects, it was necessary to keep track of the individual therapist(s) providing treatment to each patient. The therapist names were recorded and each therapist was assigned a unique number. The unique number was used for all of the subsequent analyses. The current study sought only to account for variance in patient outcomes due to therapists, not to identify or single out above average or below average therapists. 


\section{Data Analysis}

This study used multilevel modeling (MLM) to analyze all data. MLM is uniquely suited for the current study for a number of reasons. It is a primary means of analyzing nested, longitudinal data. In more commonly used statistical techniques in clinical research such as $t$ tests and analysis of variance, data are assumed to have been obtained in independent observations. However, data obtained from psychotherapy outcome research violate this assumption and are said to be nested. When data are nested, observations are related to one another in some way. The most common form of nested data in psychotherapy outcome research is when multiple observations over time belong to the same patient. MLM is able to account for this fact. The dataset was also unbalanced. MLM handles both of these situations well (Bickel, 2007). The average number of measurements per patient was 6.7 while the interval between measures was about two weeks. The preliminary analyses verified that MLM is an appropriate statistical procedure to analyze the data from this study.

The data analysis procedure followed methods described by Singer and Willett (2003) and Peugh (2010). Peugh (2010) outlines a number of steps for conducting a multilevel model including picking a parameter estimator, determining whether multilevel modeling is needed, building each level of the model, reporting multilevel effect sizes, and testing competing models using the likelihoods ratio test.

Preliminary Analyses. The preliminary statistical analyses for this study involved three main steps. First, the researcher created an unconditional model (i.e., a one-way random effects ANOVA), which included no predictors at either level. This enabled the researcher to calculate the intraclass correlation coefficient and design effect for each level to determine whether MLM was an appropriate statistical procedure. Please see Appendix B for a more detailed description 
of this procedure as well as the results from the preliminary analyses. Second, the shape of the slope for the MLM model was tested. Three possible slopes were tested: linear, quadratic, and logarithmic. These three potential slopes were chosen based on prior research on the average trajectory of PTSD symptom reduction in CPT (Macdonald, Monson, Doron-Lamarca, Resick, \& Palfai, 2011; Schumm, Walter, \& Chard, 2013). An additional preliminary step involved determining which variables would be fixed across participants (i.e., fixed effects) and which variables would be allowed to vary across participants (i.e., random effects). Once the measurement model was created, the significance of fixed effects addressing the hypotheses of interest in this study was evaluated.

Summary of Models Tested. Model A tested for main and interaction effects for completion status, initial PTSD symptom severity, and age. Model B was a simplified model based on the results of Model A. In Model B, the following non-significant parameters were removed: all quadratic interaction terms, both the main effect and linear interaction term for age. Model $\mathrm{C}$ tested for main and interaction effects for treatment type. Model D tested for main and interaction effects for African American and Hispanic status.

Parameter Estimation and Model Assumptions. Two parameter estimators are available: full information maximum likelihood (FIML) and restricted maximum likelihood (REML). Singer and Willett (2003) describe the advantages and disadvantages of each. For this study, both parameter estimators were used at various stages of the analysis based on recommendations by Hox (2010). REML was used to run the unconditional means models and when the average trajectory of the model was being determined. FIML was used to evaluate the significance of fixed effects, to make comparisons of nested models using the likelihood ratio test, and to arrive at a final multilevel model for the study. Residuals were assumed to be 
uncorrelated in all analyses. When fixed effects were being tested, the linear component of time was included as a random effect while all other effects were fixed.

Evaluation of Models. An analysis of the results of these models including all parameter estimates (for both fixed and random effects) and the significance of each parameter were used to determine which variables should be kept in the model and which variables could be removed. For all parameter estimates, a $p$-value of .05 or below was considered statistically significant. Model fit statistics included the deviance statistic, Akaike's Information Criterion (AIC), and Schwart's Bayesian Information Criterion (BIC). For all of these statistics, lower values indicate better model fit to the data. The likelihood ratio test (Singer and Willett, 2003) uses a chi-squares test to determine whether nested models provide a significantly better fit to the data. This statistic is calculated by finding the difference in the deviance statistic between two nested models, which is assumed to follow a chi-square distribution. The difference in the number of parameters estimated becomes the degrees of freedom. Models that had a deviance that was significantly lower, as indicated by a chi-square significance test, were kept, while models with significantly higher deviance were discarded.

Prior to addressing Hypothesis 2, demographic and treatment variables of interest for the CPT and PE groups were compared using chi-squares tests and independent samples $t$-tests. Independent $t$-tests had a family-wise alpha of .05 (individual $p$-value of .0125). All $p$ values were two-tailed. Post-hoc analyses for additional variables of interest were conducted via Analysis of Variance (ANOVA) using Bonferonni's correction.

Post-hoc analysis. A post-hoc analysis determined the size of therapist effects in the present sample. During the chart review, the individual therapist who provided PE or CPT was recorded and linked to each patient. Each therapist was assigned a unique identification number. 
This variable was included in the post-hoc analyses as a level 3 identifier. During this post-hoc analysis, the researcher created a three-level hierarchical model: repeated measures over time (level 1) nested with individual patients (level 2) who were nested within therapists (level 3). 


\section{Chapter IV}

Results

Two main research questions guided this study. The first research question involved determining the absolute and relative effectiveness of cognitive processing therapy (CPT) and prolonged exposure (PE). The second research question involved determining which patientlevel variables were significantly related to changes in self-reported PTSD symptoms over time (i.e., the trajectory of symptoms). Based on the prior literature on trauma-focused therapy, four hypotheses were developed to answer these questions. This chapter reviews the preliminary analyses including the determination of the hierarchical structure of the model, a justification for the multilevel modeling (MLM) procedure, and a determination of the shape of the slope. The chapter then addresses the research hypotheses of this study.

\section{Preliminary Analyses.}

Multiple preliminary statistical analyses were required prior to addressing the main research questions in this study. Results from the preliminary analyses are presented in Appendix A. The results from the preliminary analyses are presented in Appendix B. Please refer to these appendices for a more detailed description of the preliminary analyses from this study.

The results from the preliminary analyses showed that MLM was an appropriate statistical procedure to analyze the study's data and that the structure of the data was appropriate for a two-level hierarchical model (Level $1=$ time; Level $2=$ patients). About two-thirds of the variance in PTSD symptoms was due to patients (level 2) while one-third of the variance in PTSD symptoms was due to time (level 1). The preliminary analysis also showed that the shape of the average growth trajectory was quadratic. This implied that patients experienced a more rapid reduction in PTSD symptoms early in therapy. The amount of decrease from session to 
session leveled off over time. The model was not able to converge when both the linear and quadratic slope terms were included as random effects. In other words, the MLM was able to account for the fact that different patients experienced a different rate of PTSD symptom reduction. However, the MLM model would not converge unless the amount that PTSD symptoms leveled off (i.e., the quadratic component of the slope) was held constant across all patients.

Table 5 below shows the evaluation of fixed effects, which addressed the main hypotheses of the current study. 


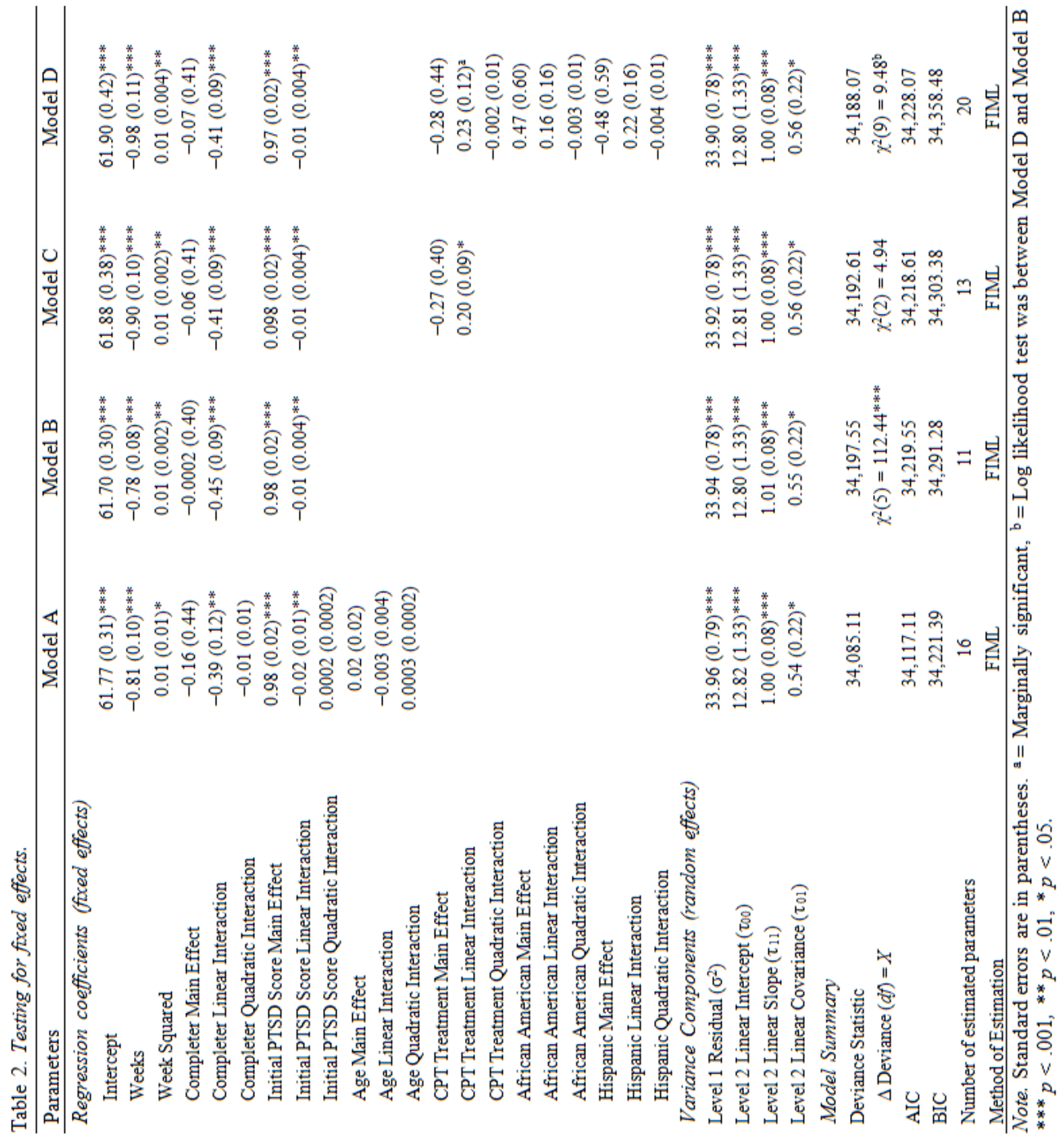




\section{Research Question 1. What is the absolute and relative effectiveness of PE and CPT?}

Hypothesis 1: Both treatments will result in a significant reduction in PTSD symptoms over time.

Models A and B tested whether CPT and PE led to significant reductions in PCL scores over time. Model A tested the significance of main and interaction effects for time, completion status, initial PTSD symptom severity, and age. Based on the results from Model A, nonsignificant fixed effects were removed to create a more parsimonious model with model B (i.e., all quadratic interaction terms, both the main effect and linear interaction term for age). Model B was retained because it was a more parsimonious model.

In Model B, the average self-reported PCL score at Session 1 was 61.70, $(B=61.70, S E$ $=0.30, p<.001)$. Participants in CPT and PE experienced a statistically significant decline of 0.78 points on the PCL each week $(B=-0.78, \mathrm{SE}=0.08, p<.001)$. This parameter represents the average rate of PTSD symptom reduction per week after taking into account additional patient-level variables such as session attendance and initial PTSD symptom severity. The quadratic interaction term for time was small, but statistically significant $(B=0.01, S E=0.002, p$ $=.002$ ). Thus, the rate of PTSD symptom reduction for both therapies is higher in the beginning of treatment and levels off as treatment progresses. The model predicts that PCL scores decline by 0.77 points between session 1 and session 2 . In comparison, the model predicts that PCL scores decline by 0.57 points between session 11 and session 12 . Thus, the leveling off of treatment gains is statistically significant, but small. Furthermore, the multilevel model included the quadratic term as a fixed effect, so it was held constant for all veterans.

The results showed that there was a significant drop in self-reported PTSD symptoms over time. Hypothesis 1 was upheld. 
Research Question 2. Which patient-level variables are significantly related to the average growth trajectory of each treatment?

Hypothesis 2: Both treatments will be equally effective. That is, there will be no difference between treatment groups with respect to the rate of symptom improvement, irrespective of pre-treatment PTSD symptom severity.

Before Hypothesis 2 was tested, participants and CPT and PE were compared with respect to demographic and treatment variables using chi-squares tests and independent sample $t$ tests. There were no significant differences between the two treatment groups with respect to age, Caucasian status, Hispanic status, or percent service connection. Women were significantly more likely to receive CPT, $\chi^{2}(d f=1, N=750)=10.24, p=.002$. African Americans were significantly more likely to receive PE, $\chi^{2}(d f=1, N=750)=7.85, p=.006$. There were no significant differences between the two treatment groups with respect to baseline total PCL score or total number of sessions attended. However, patients in PE were significantly more likely to be defined as treatment completers, $\chi^{2}(d f=1, N=750)=20.52, p<.001$, likely due to the different session requirements $(\mathrm{PE}=8 ; \mathrm{CPT}=12)$ for each treatment.

Models C and D tested the relative effectiveness of CPT and PE. Model C included main and interaction effects for CPT versus PE. Model D went one step further and also tested whether African American and Hispanic veterans had significantly different initial PCL scores and PTSD symptom trajectories when compared with Caucasian veterans. In Model C, there was no significant difference in initial PTSD symptom severity between the two treatments $(B=$ $-0.27, S E=0.40, p=.504)$. There was a statistically significant Treatment $\mathrm{X}$ Time interaction in Model C, $(B=0.20, S E=0.09, p=.027)$. This suggests that participants in CPT had a 
significantly smaller rate of decrease in PCL scores than patients who participated in PE. This interaction is plotted below in Figure 1.

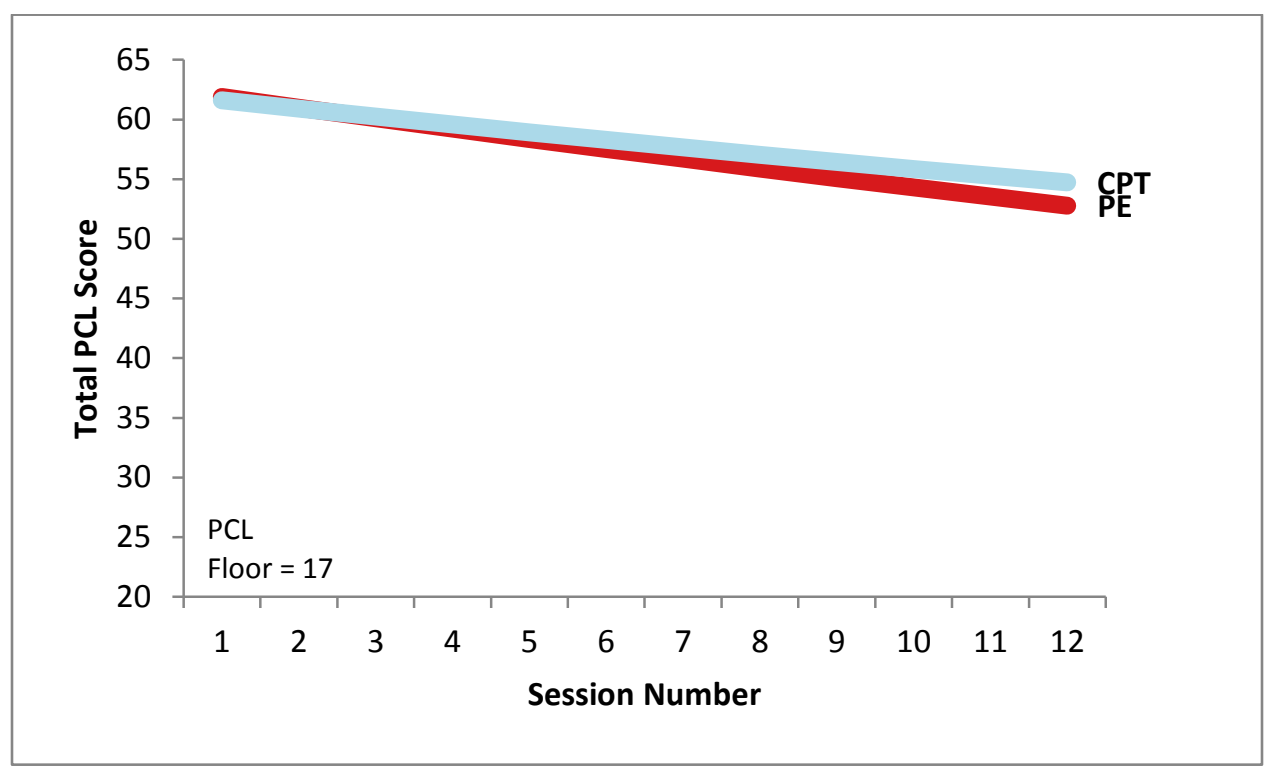

Figure 1. Treatment by time interaction.

Model C predicts that after 12 sessions, patients who participated in CPT had a total PCL score that was 2 points higher than patients who participated in PE. However, the results of the likelihood ratio test comparing Model C to Model B showed that including the Treatment X Time interaction did not result in a significantly lower deviance statistic, $\chi^{2}(d f=2, N=750)=$ $4.94, p>.200$. An examination of the random effects components showed that including the Treatment X Time interaction in Model C did not explain any additional Level 1 or Level 2 variance in the model. The Level 1 residual variance for Model B $\left(\sigma^{2}=33.94\right)$ and Model C $\left(\sigma^{2}=\right.$ 33.92) were nearly identical. The Level 2 slope variance for Model C $\left(\tau_{11}=1.01\right)$ and Model D $\left(\tau_{11}=1.00\right)$ were also nearly identical. Furthermore, the inclusion of minority status in Model D caused the Treatment $X$ Time interaction to no longer be statistically significant $(B=0.23, S E=$ 
$0.12, p=.053)$. These results suggest that the Treatment $\mathrm{X}$ Time interaction is a statistical artifact.

A post-hoc analysis of the pre- and post-treatment PCL scores for treatment completers offered additional support that both treatments were equally effective. The percentage of participants with a total PCL score below 50 before treatment was $19.6 \%$ for CPT and $17.2 \%$ for PE. At the final session, $65.5 \%$ of CPT patients and $60.8 \%$ of PE patients had total PCL scores below the diagnostic threshold for PTSD. Thus, the level of PTSD symptom severity was equivalent across both treatments and a similar percentage of patients in both treatments fell below the diagnostic threshold for PTSD by the final session.

It was concluded that Hypothesis 2 was upheld. Due to this finding, the Treatment X Time interaction was not included in the final model.

\section{Hypothesis 3:}

The following patient-level variables are predicted to be significantly correlated with the growth trajectory of PTSD symptoms: session attendance, initial PTSD symptom severity, and age at beginning of treatment.

This hypothesis was tested by Model A and Model B. Model A tested whether session attendance, initial PTSD symptom severity, and age were significantly related to the trajectory of PCL scores. Fixed effects that were not significant in Model A (including all quadratic interaction terms and all effects for age) were removed for Model B. The researcher concluded that Model B was the final model. Unless otherwise noted, all below results cite parameter estimates from Model B.

Session attendance. There was no significant difference in initial PTSD symptom severity between treatment completers and treatment non-completers $(B=-0.0002, \mathrm{SE}=0.40, p$ 
$=.999$ ). A post-hoc analysis removed the initial PTSD symptom severity variables from Model B and confirmed that this potential main effect was not masked by the presence of initial PTSD symptom severity in the model $(B=0.57, p=.546)$. There was a significant Completion Status $\mathrm{X}$ Time interaction $(B=-0.45, S E=0.09, p<.001)$. This interaction is plotted below in Figure 2 .

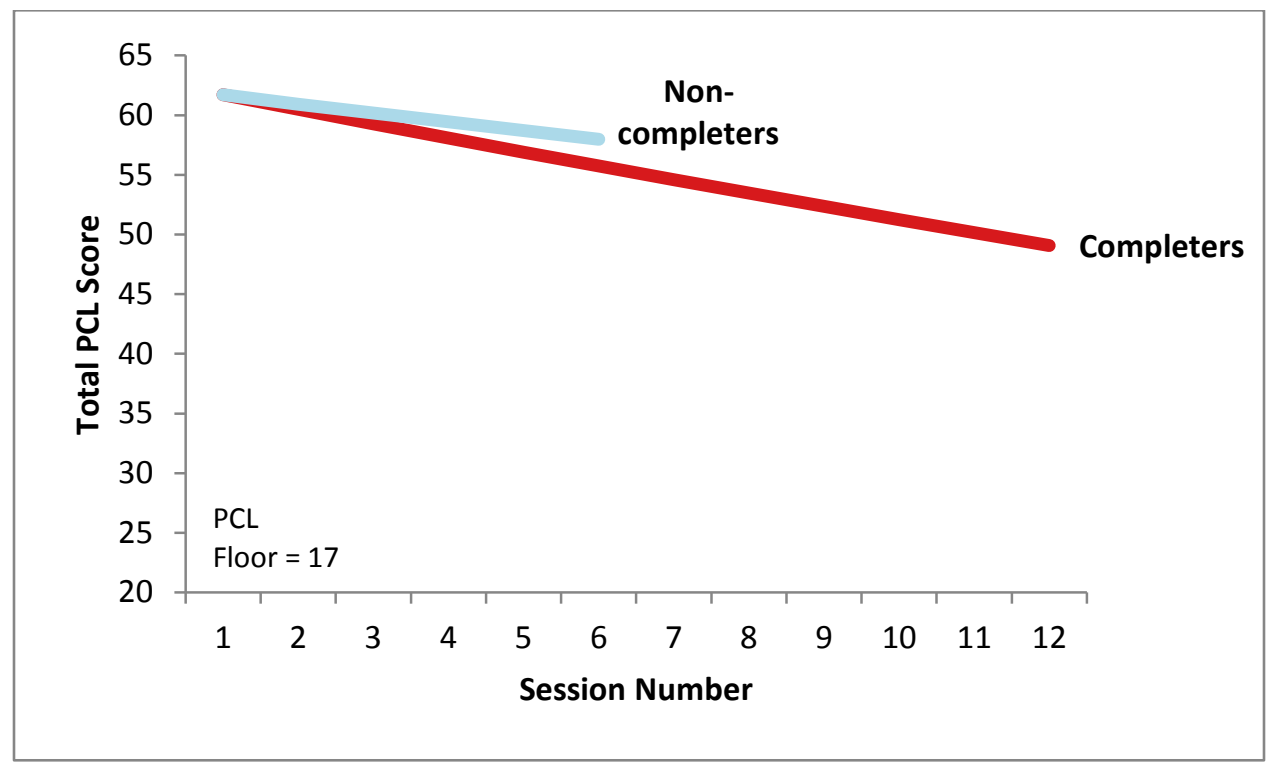

Figure 2. Completion status by time interaction. $(N=750)$ Due to the absence of a significant Treatment X Time interaction, results for CPT patients $(N=376)$ and PE patients $(N=374)$ in the above figure are combined. Treatment completers $(N=357)$ attended a mean of 11.95 sessions $(S D=3.02)$. Treatment non-completers $(N=393)$ attended a mean of 5.59 sessions $(S D$ $=5.53$ ). The above figure rounds mean session attendance for both groups to the nearest session.

The rate of PTSD symptom reduction was significantly higher for patients who completed treatment. Treatment completers experienced an average reduction of 12.63 points on the PCL over 12 sessions. This represents an average drop of $20.5 \%$ from pre-treatment PTSD symptom levels. Treatment non-completers experienced an average reduction of 3.71 points on 
the PCL after 6 sessions (the mean number of sessions attended by treatment non-completers). By session 6, patients who would later go on to complete treatment had experienced a decrease of 5.95 points, nearly twice the amount of symptom reduction as treatment non-completers. This represents an average drop of $7.15 \%$ from pre-treatment PTSD symptom levels. Notably, the self-reported PTSD symptoms for non-completers did not increase. It was concluded that session attendance as operationalized by completion status was a significant predictor of self-reported PTSD symptoms.

Initial PTSD Symptom Severity. There was a significant main effect for initial PTSD symptom severity $(B=0.98, S E=0.016, p<.001)$. This variable was mean-centered, so a main effect close to 1.000 was expected. There was also a significant Initial PTSD Symptom Severity $\mathrm{X}$ Time interaction $(B=-0.01, \mathrm{SE}=0.004, p<.001)$. This interaction is plotted in Figure 3 below.

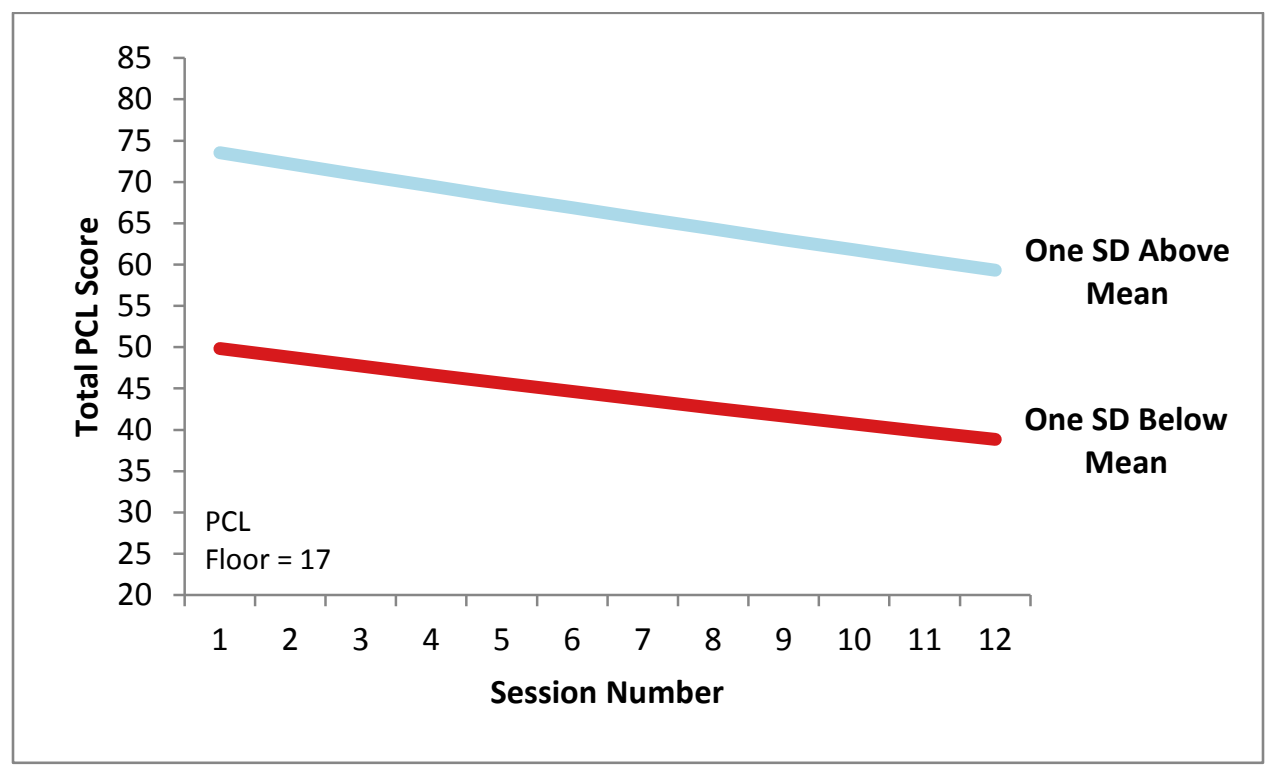

Figure 3. Initial PTSD symptom severity by time interaction. Figure uses parameter estimates from Model B. This interaction is plotted for treatment completers $(N=357)$. Due to the absence 
of a Treatment $\mathrm{x}$ Time interaction, results for CPT patients $(N=148)$ and PE patients $(N=209)$ in the above figure are combined.

On average, patients with higher initial self-reported PTSD symptoms tended to experience a larger decrease in symptoms over the course of the therapy. Despite this significant interaction, the results showed that veterans at varying levels of PTSD symptom severity all benefited from trauma-focused psychotherapy. It was concluded that although the Initial PTSD Symptom Severity x Time interaction was statistically significant, all veterans regardless of initial PTSD symptom severity benefited from trauma-focused therapy.

Age. Preliminary analyses showed that there was a significant, positive correlation between Age and Initial PTSD Symptom Severity, $r(748)=0.13, p<.001$. However, the results from Model A showed that there was no significant main effect for age $(B=0.02, S E=0.01, p=$ .221). The Age $\mathrm{x}$ Time interaction was also not statistically significant $(B=-0.004, S E=0.004$, $p=.329)$. Thus, including the main effect and interaction for initial PTSD symptom severity resulted in non-significant parameter estimates for age. It was concluded that age differences in initial PTSD symptom severity were masked by the inclusion of additional covariates in the model. Encouragingly, the absence of an Age x Time interaction suggests that all veterans regardless of age benefit from $\mathrm{CPT}$ and PE.

In summary, Hypothesis 3 was partially upheld. There was a large interaction due to session attendance. Initial PTSD symptom severity led to a small but statistically significant interaction. Age was not significantly related to initial PTSD symptoms or to the average growth trajectory of PTSD symptoms. However, the main effect of age was masked by including initial PTSD symptom severity in the model. 


\section{Hypothesis 4:}

The trajectory of PTSD symptoms for Caucasian, African American, and Hispanic veterans is predicted to be identical, irrespective of pre-treatment differences between racial/ethnic groups with respect to PTSD symptom severity.

This hypothesis was tested in Model D by including binary variables to test for main effects and interactions for African American and Hispanic status.

In Model D, there was no significant main effect for African American veterans $(B=$ $0.47, S E=0.60, p=.438)$ or Hispanic veterans $(B=-0.48, S E=0.59, p=.413)$. In Model D, there was no significant difference between Caucasian, African American, and Hispanic patients with respect to initial PTSD symptom severity. The African American X Time interaction was not statistically significant $(B=0.16, S E=0.16, p=.344)$. The Hispanic X Time interaction was also not statistically significant $(B=0.22, S E=0.16, p=.179)$. None of the quadratic interaction terms were statistically significant. Furthermore, the likelihood ratio test comparing Model D with the final model showed that including the variables for African American status and Hispanic status did not improve the accuracy of the model, $\chi^{2}(d f=9, N=750)=9.479, p>.200$ Thus, the average trajectory of PTSD symptom reduction was equivalent across Caucasian, African American, and Hispanic veterans. A main effect for race/ethnicity has been found in prior research (e.g., Tuerk et al., 2011). To account for the fact that including initial PTSD symptom severity may have masked a main effect for racial/ethnic minority veterans, a post-hoc One-Way ANOVA with Bonferonni's correction compared the initial PTSD symptom severity among Caucasian $(M=60.46, S D=12.35)$, African American $(M=62.68, S D=$ 12.154), and Hispanic veterans $(M=63.78, S D=11.50)$. There was a significant difference between the groups, $F(2,685)=4.40, p=0.013$. Post-hoc analyses revealed that Hispanic 
veterans had significantly higher baseline PCL scores than Caucasian veterans, $p=.021$. There was no significant difference in initial PCL scores between African American veterans and Caucasian veterans $(p=.223)$ or between Hispanic veterans and African American veterans ( $p=$ .999). Thus, including initial PTSD symptom severity in the model masked the finding that Hispanic veterans tend to begin therapy with significantly higher PCL scores. However, the nonsignificant interactions between minority status and time in Model D indicated that veterans of all racial/ethnic groups recover at similar rates during treatment. Hypothesis 4 was partially upheld.

\section{Post-hoc Analyses.}

Therapist Effects. Details regarding the post-hoc analysis of therapist effects are included in Appendix C. Therapists accounted for a significant amount of the variance in PCL scores, Intraclass Correlation Coefficient $=0.065, p=0.024$. The design effect for therapists was 1.70. Some researchers believe that a design effect estimate of greater than 2.00 is needed for MLM (Muthen, 1991, 1994; Muthen \& Satorra, 1989, 1995). These results indicated that although the amount of variance in PCL scores due to therapists was statistically significant, the structure of the data did not allow for the creation of a three-level hierarchical model. 


\section{Chapter V}

\section{Discussion}

This chapter summarizes and offers an interpretation of the main findings of this study by reviewing the results from the preliminary analyses and main research hypotheses. The chapter then discusses how the results from the current study fit within the prior literature on traumafocused therapy in the general discussion section. The limitations of this study, future directions for new research, and implications for practitioners of CPT and PE are then discussed.

\section{Summary of the Findings}

Preliminary Analyses. One notable finding of the preliminary analysis was that there was a high amount of variance in self-reported PTSD symptoms due to patient-level factors. In other words, the outcome experienced in therapy was most related to patient-level factors rather than due to the treatment itself. There are many potential patient-level factors that may influence treatment outcomes. Patient-level factors that may be significantly related to treatment outcomes include actual differences in the severity of PTSD among patients, different levels of participation in treatment, and different rates of homework completion. Various extra-therapeutic factors such as different personal histories, trauma histories, and ongoing life circumstances are also likely important components. Variations in self-reported PTSD symptoms may also be the result of errors of measurement, which includes different response styles that patients may have while filling out self-report measures. However, it was encouraging that the results showed that MLM is an appropriate procedure to model the change in self-reported PTSD symptoms over time.

The finding that the shape of the growth trajectory is quadratic is notable because it indicates a more rapid reduction in PTSD symptoms during the initial sessions. However, this 
finding was exploratory, was not hypothesized beforehand, and thus emerged from the data. This finding means that patients generally experience more rapid declines in self-reported PTSD symptoms in the first few sessions while declines over the course of therapy level off in subsequent sessions. This finding hints that multiple mechanisms of change may be at work in trauma-focused therapy. In CPT, the full trauma account (i.e., the part of the treatment during which the patient writes a detailed account of his or her trauma including all sensory details as well as his or her thoughts and feelings related to the event) is not assigned until after the third session. In PE, patients begin their participation in imaginal exposures during the third session and participate in in vivo exposures after the second session. However, in practice, many therapists conduct the second session of PE over two weeks due to the high amount of material to be covered. Thus, the initial reduction in self-reported PTSD symptoms in both treatments occurs before the active ingredients (cognitive restructuring in CPT and exposure in PE) have had time to work. The topics covered in both treatments during the first two to three weeks are largely psychoeducational. Many of the tasks therapists engage in involve establishing a strong therapeutic relationship with the patients, communicating the rationale for the treatment, and instilling hope that the patient can recover. Thus, the more rapid initial gains in both treatments may be due to the influence of the common factors. However, the current study was not designed to empirically address that question.

Hypothesis 1. It was predicted that both $\mathrm{CPT}$ and PE would result a significant reduction of PTSD symptoms over time. As predicted, Hypothesis 1 was confirmed. The results showed that participants in both treatments experienced a significant reduction in PTSD symptoms.

Percent change is one way of quantifying response to treatment in psychotherapy outcome research. Due to the use of multilevel modeling, the present study did not involve the 
calculation of traditional effect sizes (i.e., Cohen's D). Recall that on the PCL, veterans rate the frequency they experience 17 PTSD symptoms on a likert scale ranging from 1 (not at all) to 5 (extremely). Responses on the 17 items are summed to derive a total PCL score. This score is an indicator of PTSD symptom severity. A decline in total PCL scores indicates an improvement in PTSD symptoms, that veterans experience PTSD symptoms less frequently. This is a desirable outcome for both CPT and PE. Percent change is calculated by finding the difference between the initial PCL score at session 1 and the final PCL score at the end of therapy. Then, that value is divided by the initial total PCL score to find the percent change in total the PCL score due to participation in therapy.

After taking into account completion status and initial PTSD symptom severity, participants experienced a drop of about 0.8 points on the PCL per session. Patients who attended the average number of sessions (about 8) had a decline in self-reported PTSD symptoms of between 5 and 6 points. This represents a 10 percent decline in total PCL scores when compared to baseline. This decline falls above the threshold of 5 points needed to indicate a favorable response to treatment (National Center for PTSD, 2014), but below the 10-point threshold that indicates clinically significant change. Thus, patients who participated in CPT and PE responded favorably to treatment. However, treatment gains were predicted to approach clinically meaningful levels only when a full therapy protocol was completed.

Hypothesis 2. It was predicted that both treatments would be equally effective. As predicted, Hypothesis 2 was confirmed. There was no significant difference in baseline total PCL scores between the CPT and PE groups. Furthermore, the rate of the decline in PTSD symptoms was equivalent between the two treatments. Both treatments led to a significant increase in the percentage of veterans whose total PCL score were below the diagnostic cutoff for PTSD. These 
results indicated that both CPT and PE were equally effective treatments. This result lends support to the VA's promotion of both CPT and PE as evidence-based treatments for PTSD.

Hypothesis 3. It was predicted that session attendance, initial PTSD symptom severity, and age at beginning of treatment would be significantly correlated with the trajectory of PTSD symptoms throughout treatment. The results for Hypothesis 3 were mixed. There was no significant difference in initial PTSD symptom severity between patients who did and who did not complete the treatments. As predicted, treatment completion was associated with a significantly higher rate of PTSD symptom reduction. Patients who completed 12 sessions were predicted to experience a decline of 13.7 points on the PCL, which falls above the 10 point threshold needed for clinically significant change (National Center for PTSD, 2014). This represents an average decline of 22 percent from the average baseline total PCL score. Patients who completed the average number of sessions $(N=8)$ were predicted to have a 9.3-point decline in their total PCL score, which represents a 15 percent reduction in symptoms. This value is well above the 5-point threshold indicative of favorable response to treatment. The results of the model showed that patients who participated in 9 or more sessions were likely to experience a clinically significant reduction in PTSD symptoms.

Completion status was one of the most important predictors of PTSD symptom reduction. Patients who completed the full treatment protocols experienced significantly better outcomes (i.e., a greater reduction in their total PCL score) than patients who dropped out of treatment. Patients who completed 6 sessions (the average number of sessions for patients who dropped out of therapy) experienced a decline of 4.4 points on the PCL. This value is below the 5-point cutoff that is used to indicate a favorable response to treatment (National Center for PTSD, 2014). 
The influence of initial PTSD symptom severity was statistically significant. According to this finding, patients who had more severe initial PTSD symptoms were predicted to have a larger decrease in PTSD symptoms. Take, for example, a hypothetical group of patients who completed 12 sessions of trauma-focused psychotherapy. Patients who began treatment with a total PCL score of 74 (one standard deviation above the mean) were predicted to have a 30 percent decline in their total PCL score by the end of therapy. Patients who began treatment with a total PCL score of 50 points (one standard deviation below the mean) were predicted to have a 22 percent decline in in their total PCL score by the end of treatment. The decline in PCL scores across all both groups is clinically significant. Despite the interaction, patients in all three groups benefited from treatment. Indeed, patients who began treatment with a total PCL of about 50 points who completed treatment were predicted to have total PCL scores well below the 50-point diagnostic threshold for PTSD. Thus, although this interaction is statistically significant, it is unlikely to be clinically significant.

The non-significant finding regarding age was unexpected, especially in light of the preliminary finding that age and initial PTSD symptom severity were positively correlated. It is likely that this is due to the inclusion of initial PTSD symptom severity in the model. After accounting for initial PTSD symptom severity, increased age was not associated with significantly higher PTSD symptoms.

Hypothesis 4. It was predicted that the trajectory of PCL scores reported by African American and Hispanic veterans would not be significantly different than the trajectory of PCL scores by Caucasian veterans. The results confirmed hypothesis 4 . These results suggest that both CPT and PE are equally effective for Caucasian, African American, and Hispanic veterans. 


\section{General Discussion}

When the results of the present study are compared to the prior research on traumafocused therapy, an encouraging picture emerges regarding the effectiveness of CPT and PE.

Quadratic slope. The present study's finding that the shape of the average trajectory of PTSD symptoms was quadratic is consistent with prior research on both CPT and PE. Schumm, Walter, and Chard (2013) also found that the self-reported PTSD symptoms of patients in CPT followed a quadratic trajectory. Tuerk et al. (2011) had similar findings with a sample of veterans who received PE. The present study extends this finding to a larger, more diverse sample. According to the final multilevel model, it appears that patients in both treatments experience rapid initial declines in self-reported PTSD symptoms, which then level off over time. It should be noted that prior studies have shown that approximately 10 percent of patients may experience an exacerbation following the first imaginal exposure in PE, when patients are asked to describe in detail what happened during their traumatic events (Foa, Zoellner, Feeny, Hembree, \& Alvarez-Conrad, 2007). This exacerbation in symptoms was not observed in the present study. However, the present study was not designed to address that question. Patients who have ignored or avoided their PTSD symptoms as a form a symptom management may experience an initial increase in PTSD symptoms, especially when they first begin confronting trauma reminders. Encouragingly, Foa et al. (2007) found that patients who experienced an exacerbation in their PTSD symptoms experienced similar benefits from PE when compared to patients who did not report an exacerbation in symptoms. Their findings have not been confirmed for CPT patients. Furthermore, this finding was observed in non-combat, female victims of sexual assault. While additional research could be done in this area, it is encouraging 
that most veterans appear to report rapid initial gains while participating in trauma-focused therapy.

Comparing present findings to prior research. The average initial total score on the PCL found in the present study was 61.7. This finding is equivalent to the total PCL score at pretreatment found in prior studies. Previous estimates of the initial total PCL score in studies with veterans have ranged from 56.7 (Jeffreys et al., 2014) to 65.5 (Gilman, Schumm, \& Chard, 2012). Thus, patients in the present study tended to begin treatment with a similar level of PTSD symptoms as in prior studies. The significant decrease in PTSD symptoms over time reported by patients who participated in CPT and PE was highly consistent with prior research. All prior randomized clinical trials of CPT (e.g., Monson et al., 2006;) and PE (e.g., Foa et al., 2005) have found that patients who receive either treatment tend to to experience a reduction in self-reported PTSD symptoms. The current study's results also align with prior research that included veterans receiving care in conditions more reflective of actual clinical practice (e.g., Chard et al., 2012; Jeffreys et al., 2014, Tuerk et al., 2011;).

When one compares the results in the present study to past research on CPT and PE, it becomes clear that the observed response to treatment in the present study is less than what has been observed in prior studies. Percent reduction is operationalized as the percent drop of total PCL scores between baseline and the final session. After controlling for the number of sessions attended and initial PTSD symptom severity, treatment completers in the present study experienced an average reduction of 20 percent in CPT and 22 percent in PE. The percentage of PTSD symptom reduction reported by treatment completers in prior studies has been higher. For example, Monson et al. (2006) found that patients who received CPT experienced an average reduction on the PCL of over 26\%, although they received therapy under conditions with a 
higher amount of experimental controls. Interestingly, prior studies where patients received treatment under conditions that were more reflective of clinical practice have also found larger treatment effects. Chard et al. (2012) found that patients who completed CPT experienced an average reduction of $29.5 \%$ in total PCL scores. Jeffreys et al. (2014) found that patients who completed PE experienced an average reduction of over $40 \%$ in total PCL scores. Tuerk et al. (2011) also found that veterans who completed PE experienced an average reduction of $40 \%$ on the PCL.

Potential explanations for reduced treatment effects. There are multiple potential reasons why the amount of symptom reduction reported by recipients of CPT and PE was diluted in the present study. One potential explanation is that the patients in the present study received a lower dosage of both therapies. Patients in the present study attended an average of 8.5 sessions for CPT and 8 sessions for PE. In contrast, patients in past studies of CPT and PE have tended to receive closer to 11 or 12 sessions of treatment.

Treatment effects in psychotherapy outcome research also tend to be diluted in conditions where there are fewer experimental controls (Kazdin, 1998). Estimates of treatment effects tend to be higher when samples are more homogenous. Conditions in the present study were more reflective of conditions encountered in the realm of current clinical practice. As a result, the sample was highly heterogeneous, which tends to reduce observed treatment effects. Any patients who mental health providers felt would benefit from CPT or PE were included in the study. Patients treated by providers of varying levels of training ranging from psychology and social work trainees to highly experienced doctoral-level therapists were included in the present study. Data also were collected from multiple VA medical centers as well as their associated community-based outpatient clinics. In contrast, prior studies on the effectiveness of CPT and 
PE, even those that analyzed data from patients in conditions reflective of actual clinical practice, tended to have higher levels of control than the present study. In general, mental health providers in past studies were either restricted to doctoral level clinicians (e.g., Monson et al., 2006), were experienced members of clinical teams (e.g. Tuerk et al., 2011), or were receiving clinical training in CPT or PE concurrently to their clinical work (e.g., Chard et al., 2012). Much of the past research on CPT and PE has also taken place in single treatment sites rather than multiple locations.

There is an additional methodological difference between the present study and past studies that may be an important factor. Chard et al. (2012) and Jeffreys et al. (2014) both utilized a pre-post design in which they used multiple imputation to estimate missing posttreatment scores. Multiple imputation is a commonly used practice for missing data which has a high degree of support in the literature (e.g., Wayman, 2003). The estimate of treatment effects in the present study was calculated by observing the longitudinal reduction in symptoms reported by treatment completers using multilevel modeling. It appears that both methods may lead to different estimates of treatment effects. However treatment effects are calculated, it is encouraging that both CPT and PE are highly effective. Considering all of these factors, it is not surprising that the treatment effects were diluted in the present study. However, it is clear that despite losing some of their potency, both CPT and PE remained effective treatments for PTSD when practiced under conditions highly reflective of clinical practice.

Relative effectiveness of CPT and PE. With respect to the relative effectiveness of PE over CPT, there have been some conflicting findings in the literature. In the only randomized clinical trial that compared both treatments, Resick et al., (2002) found that CPT and PE led to equivalent outcomes. However, Jeffreys et al. (2014) concluded that PE was significantly more 
effective than CPT in a large sample of over 500 veterans after controlling for age, service era, and race/ethnicity. The current study failed to replicate Jeffreys et al.'s (2014) finding. There was a small, but non-clinically significant difference in effectiveness between the two treatments in the present study. Both Jefferys et al. (2014) and the present study utilized a retrospective chart review with a large sample of veterans, so the present study may help to shed some light on this contradiction.

In the present study, there was a statistically significant difference between CPT and PE after controlling for initial PTSD symptom severity and completion status. However, this interaction was no longer statistically significant after controlling for race/ethnicity. Furthermore, for treatment completers the statistically significant interaction is predicted to result in a difference of about two points in the total PCL scores between treatments. This difference between treatments, though statistically significant, is unlikely to be clinically meaningful. Inferring a significant difference between treatments when there was a lack of randomization of patients between treatments also seems unwarranted given the potential for selection bias between treatments. The fact that female veterans were more likely to receive CPT and that African American veterans were more likely to receive PE in the present study suggests that there are additional, unseen selection biases in the present sample. Assignment to treatment groups in the present study was not random. Patient-level factors such as differences in trauma type (e.g., combat versus MST) and trauma severity may have also resulted in the observed differences between treatments. In light of the potential selection bias in the present study and the relatively small difference observed between treatments, it seems prudent to retain the null hypothesis of there being no significant differences between treatments until additional results obtained from more controlled settings are available. 
The role of completion status. The most clinically meaningful interaction in the present study was related to whether patients completed treatment. Patients who completed treatment tended to have clinically significant declines in PTSD symptoms while patients who did not complete treatment experienced little to no change in their symptoms. This aligns with prior research that session attendance appears to be related to symptom change (Bradley et al., 2005). However, dropout rates in the present study appear to be higher than in past studies. When dropout is defined as failure to complete a full therapy protocol, $44 \%$ of patients dropped out from PE while $60.6 \%$ of patients dropped out from CPT. Recall that PE can be completed in 8 or more sessions while CPT is typically completed following 12 sessions. In a sample of over 1900 veterans who received PE, Eftekhari et al. (2013) found that $28 \%$ of patients dropped out from treatment. At least for PE, the current study's dropout rate was similar to the dropout rate of 46.8\% found by Wierzbicki and Pekarik (1993) in a meta-analysis of 123 studies. Regardless of the prevalence of dropout, it is clear that dropout remains a key area of concern for traumafocused therapy, especially given the common tendency for people with PTSD to engage in avoidance, which may directly impede progress in trauma-focused therapy.

The role of initial PTSD symptom severity. Due to the relative paucity of research on the relationship between initial PTSD symptom severity and the trajectory of PTSD symptom reduction during treatment, the finding that there is a significant interaction between the initial PCL score and time has relatively few precedents in the literature. It should be noted that the interaction was small and unlikely to be clinically meaningful in the present study. Jeffreys et al.'s (2014) results hinted that this interaction would be present. However, Schumm, Walter, and Chard's (2013) results suggest that this interaction is likely to be more complex. They found that there are three latent classes of veterans with different trajectories of PTSD symptom reduction. 
It appears that one group of veterans begins treatment with high PCL scores and reports more rapid reductions in symptoms, while a another group of veterans begins treatment with high PCL scores and experiences a slower rate of decline in their symptoms. Across studies, it is encouraging that the evidence shows the effectiveness of both treatments regardless of initial PTSD symptom severity.

The role of age. The present study's finding regarding the relationship between the age of the participants and the reduction in PTSD symptoms is less clear than other findings in the study. Older veterans did have significantly higher self-reported PTSD symptoms, but that main effect was not present in the final multilevel model due to the inclusion of initial PTSD symptom severity as a separate variable. Prior studies (Chard et al., 2012; Yoder et al., 2012) have found that Vietnam and Persian Gulf War veterans have higher initial scores on the PCL than OEF/OIF/OND veterans. However, Yoder et al.'s (2012) finding that Persian Gulf War veterans who participated in CPT benefited significantly less than both Vietnam and OEF/OIF/OND veterans suggests that age is likely a proxy for other variables of interest (e.g., type and severity of trauma exposure). The current study was not designed to address this question. It does appear that when age is included as a linear variable, there is no difference in the rate reduction in PTSD symptoms across ages. However, if the relationship between age and PTSD symptom reduction is curvilinear, with Persian Gulf Veterans less responsive to treatment than Vietnam or OEF/OIF/OND veterans, then the present study would be unable to address that question without specifically comparing the outcomes of veterans of different eras. Unfortunately, the data in the present study prevented addressing that question directly because the VA combined veterans of Operation Desert Storm and OEF/OIF/OND into one category (i.e., Persian Gulf). 
Race/Ethnicity and treatment outcomes. Regarding the association between

race/ethnicity and treatment outcomes, there have been some conflicting results in the literature. Tuerk et al. (2011) found that African American veterans who participated in PE had significantly higher baseline scores on the PCL. However, they found that when compared to Caucasian veterans, African American veterans did not have significantly different outcomes post-treatment and that race was not a significant predictor of the slope of PTSD symptoms over time. Jeffreys et al. (2014) found that African American veterans who participated in PE had significantly better improvements on the PCL than other ethnicities. However, given the relatively small number of African Americans in their sample, they cautioned that their results should be interpreted tentatively. Jeffreys et al. (2014) found no significant difference in outcomes between Caucasian and Hispanic veterans who participated in CPT or PE.

The results from the present study confirm Tuerk et al.'s (2011) finding that significant differences between racial/ethnic groups in pre-treatment PTSD symptom severity are present. However, in contrast to Tuerk et al. (2011), the present study found that Hispanic veterans had significantly higher baseline PCL scores, while there was no significant difference in baseline PCL scores between Caucasian and African American veterans. There are multiple reasons why Hispanic veterans may have different initial PTSD symptoms. It should be noted that the average initial PCL score for African American veterans was higher than for Caucasians veterans, but the difference did not approach statistical significance. It is likely the case that the failure of the present study to replicate Tuerk et al.'s (2011) finding is due to differences in the samples between the studies. However, it does appear that racial/ethnic minority veterans may tend to enter therapy with significantly higher baseline PCL scores than Caucasian veterans. 
One possible explanation for this finding is that racial/ethnic minority veterans are more likely to encounter traumatic experiences as civilians (Frueh et al., 1997). Another potential explanation is higher exposure to combat conditions experienced by racial/ethnic minority service members in the Vietnam War (Kulka et al., 1990). It is also possible that racial/ethnic minority veterans with subclinical PTSD are less likely to seek out treatment due to the increased barriers they encounter while seeking mental health treatment—both institutional (e.g., relatively few providers within the VA are of minority status) and societal (e.g., stigma regarding mental health treatment). However, the results of the present study did snow that trauma-focused therapy is equally effective across racial/ethnic groups. This finding is a confirmation of Tuerk et al.'s (2011) results. The present study had one of the largest samples of African American and Hispanic veterans in the literature, so this finding is encouraging.

Unexpected findings. There were some surprising findings in the present study that deserve additional attention. The findings that female veterans were significantly more likely to receive CPT and that African American veterans were significantly more likely to receive PE were unexpected. Neither finding was hypothesized a priori, so these results should be interpreted with caution.

It is unclear why female veterans were more likely to receive CPT while male veterans were more likely to receive PE. It is abundantly clear that female veterans are significantly more likely to have experienced military sexual trauma than male veterans (Institute of Medicine, 2013). However, both CPT and PE were originally developed to treat the victims of civilian sexual assault. It is possible that perceived gender differences might result in providers being more likely to assign female veterans to CPT and male veterans to PE. Perhaps providers feel that male veterans would be less responsive to CPT? Or, if the cognitive reactions experienced 
by veterans following military sexual trauma are perceived by their mental health providers to be an especially prominent component of their presenting issues, providers may be more likely to encourage such veterans to participate in CPT.

The finding that African American veterans were more likely to receive PE is more puzzling. Subsequent research should confirm whether this finding is real or due to sample error. In other words, it is possible that the present sample is somehow not generalizable to the population of African American veterans who are receiving trauma-focused therapy across the VA system as a whole. However, if the finding does hold up, it raises some important questions as to why African American veterans are more likely to be assigned to receive PE. Does this difference reflect a preference expressed by African American patients after they are presented with an option of either CPT or PE, or are mental health practitioners more likely to recommend that African Americans participate in PE? If the latter is the case, is that due to an implicit bias held by mental health practitioners that African American patients are less able to be successful in a cognitively demanding, insight-oriented therapy such as CPT? No conclusions should be drawn at this point, but this finding does warrant further attention.

Therapist effects. Some researchers (e.g., Wampold, 2001) have argued that failing to account for that therapist effects may bias estimates of treatment effects. In other words, if therapist factors such as differences in the effectiveness of therapists, different levels of experience between therapists, and varying levels of adherence to the psychotherapy manual are highly related to treatment outcomes, ignoring therapist effects could cause researchers to overestimate the effectiveness of treatments. A post-hoc analysis estimated the size of therapist effects in the present sample to account for this potentially important variable. The researcher achieved this by creating a three-level hierarchical model with repeated measures over time 
(level 1) nested within patients (level 2) nested within therapists. To run the three-level hierarchical model, it was important to have a high number of patients per therapist. Thus, therapists who saw three or fewer patients were excluded from the three-level hierarchical model. The final sample for the three-level model consisted of 624 participants.

A main finding of the post-hoc analysis revealed that therapist effects were statistically significant. In other words, the results showed that therapist factors were responsible for a significant proportion of patient outcomes in therapy. However, the study was not designed to evaluate which therapist effects (e.g., level of experience, adherence to protocol, accuracy of empathic statements, etc.) were most highly related to patient outcomes in therapy.

The current study's estimate of therapist effects is similar to prior research, which as estimated that therapists account for between 5 percent (Wampold \& Brown, 2005) and 12 percent (Laska, Smith, Wislocki, Minami, \& Wampold, 2013) of the variance in patient outcomes. However, the present study's findings are in contrast to Elkin et al. (2006) who did not find significant therapist effects. In the present study, the results indicated that although therapist effects were statistically significant, they were not large enough to analyze using multilevel modeling. It was concluded that ignoring therapist effects did not lead to a significant level of bias in the present study.

One interpretation of this finding is that therapist effects are minimal and are not significantly related to treatment outcomes. Indeed, much of the outcome research on traumafocused therapy has been conducted under this assumption. A chief limitation in accounting for therapist effects is that one must include a high number of patients per therapist. Having a sample that meets this requirement is quite difficult. In the present study, there was a high amount of variability in the number of patients per therapist, with some therapists having as 
many as 50 patients while many other therapists had only 4 patients. Thus, the data in the present study were not well suited for an analysis that could have accounted for therapist effects. Future research of PTSD treatments would need to include even larger samples than the present study in order to provide a more accurate estimate of therapist effects.

For most researchers, such an analysis seems unfeasible. Dropping the requirements of a three-level hierarchical model enabled the inclusion of nearly 125 more participants and provided a more robust test of many of the research questions in this study. Accounting for therapist effects would have reduced the statistical power to answer additional questions of interest.

\section{Limitations}

Internal validity. Due to the fact that this is an effectiveness study, a main limitation of this study is the lack of an experimental design. Therefore, the present study lacked the types of controls are used to maximize internal validity. Patients were not randomly assigned into treatment groups. Random assignment is necessary to limit the influence of selection bias and to control for treatment gains due to maturation. The fact that there were significant differences between the treatment groups with respect to demographic variables does suggest that selection biases were present in the current study's sample. The current study also did not have a control group. Control groups enable any observed gains in therapy to be attributed to the treatments rather than due to maturation. However, multiple past studies that contained control groups have verified the absolute efficacy of both CPT and PE.

External validity. Although external validity is a strength of this study, there are limitations to the study's external validity that warrant caution when generalizing the findings to the population of veterans as a whole. 65 percent of the participants in the present study resided 
in two states (Arizona and California) and received treatment at one of four treatment locations. 35 percent of the participants received treatment in one of 8 states at one of 9 treatment sites. Veterans from some regions of the country (e.g., Appalachia, the Pacific Northwest) were not represented in the study's sample while veterans from other regions (e.g., the Northeast) were underrepresented. Thus, one should take caution when generalizing the results from the present study to veterans living in regions that were underrepresented in the present study's sample.

Unaccounted for variables. There were also multiple unaccounted for variables in the present study that may have influenced the progress that patients made. Patient-level variables that were not accounted for include pharmacotherapy data, trauma type, trauma severity, homework adherence, patient comprehension of topics covered in therapy, and motivational factors. The prevalence and severity of cognitive factors related to trauma were also not accounted for. Patient-level variables that influence one's ability to participate in psychotherapy such as SES, current income, employment status, and level of education were also not accounted for. Therapist-level variables that may have influenced progress in treatment that were not accounted for include treatment adherence, therapist allegiance to treatment, and years of experience.

Also, the current study only included one dependent variable. Having only one dependent variable provided more limited evidence for the effectiveness of the treatments. Additional constructs of interest that could have provided evidence for the effectiveness of both treatments may include depressive symptoms as measured by the BDI-II, diagnostic status as measured by a structured clinical interview, functional capacity as measured by indicators such as sustained employment, and the impressions of the patients' progress according to family members. Multiple outcomes that are measured via different methods of assessment (i.e., self-report, direct 
observation, etc.) would have provided stronger evidence for the effectiveness of CPT and PE. The conclusions from this study should be limited given that the outcome measure chosen for this study provides a limited picture of the effectiveness of both treatments. In other words, there are outcomes from participating in CPT or PE that may be just as important as PCL scores that were not included in this study.

Testing effects and limitations of self-report measures. An additional limitation of the present study involved issues related to testing and self-report measures. Taking the same test repeatedly can influence the reliability of that measurement. I am unaware of any published studies that have verified whether the PCL is measuring the same construct at session 1 and session 12. If the PCL is not measuring the same construct after repeated administrations, then the total PCL score at session 1 would have a different meaning than a total PCL score obtained at session 12. Both would need to be interpreted with greater caution. Also, the use of total PCL scores in the present study masks the fact that scores are more accurately analyzed as separate factor scores for re-experiencing symptoms, avoidance symptoms, negative alterations in cognitions and mood, and hyper-arousal symptoms. Furthermore, all data on patient progress in therapy were obtained from self-report measures. Limitations of self-report measures include biases due to social desirability and different response styles while completing the assessments, which increase measurement error.

\section{Future Directions}

There are a number of potential future issues regarding trauma-focused therapy that researchers could address in light of the findings from the present study. The finding that completion status was the most clinically relevant factor that was related to outcomes in therapy raises an important question: What factors lead to favorable versus non-favorable responses to 
treatment? Future researchers could alter both interventions to help reduce dropout rates. Potential alterations to the interventions include paying additional attention to the patients' motivational factors prior to their participation in psychotherapy, changing interventions in veterans identified as being at an increased risk for dropout to help keep these veterans in therapy, or modifying procedures in clinical trials to better keep track of patients who drop out.

In light of the contrasting results in the current research regarding the relative effectiveness of CPT and PE, additional research under more controlled conditions could confirm whether both treatments are equally effective. It would be beneficial for such research to include multiple geographic areas to help maximize the external validity of the findings. Additional research could also investigate how each cluster of PTSD symptoms responds to treatment over time. Research on the potential influence of repeated administrations of the PCL could confirm whether the instrument is measuring the same construct over the course of therapy. Alternative uses of multilevel modeling, such as the creation of spline multilevel models, may more accurately evaluate whether some patients experience an exacerbation in their PTSD symptoms during the first third of treatment.

\section{Implications for Practice}

Given the high amount of external validity in the present study, the results from this analysis have many implications for mental health practitioners who provide trauma-focused therapy. Perhaps the most encouraging finding for mental health practitioners is that the effectiveness of both CPT and PE was confirmed in conditions that are highly reflective of clinical practice. When patients are assigned to one of these treatments based on their preferences and based on the ability of their therapist to provide the treatment, patients tend to have highly beneficial outcomes. The best available data that we have suggests both treatments 
are equally effective, but that participation in one or the other treatment should be determined in light of a patient's trauma history and after consultation with each individual patient.

Furthermore, when promoting CPT and PE to potential patients, mental health providers can be confident in communicating that although participation in both treatments may cause some distress, neither treatment has been found to be harmful.

Providers can also take comfort in the finding that both CPT and PE work equally well across many subgroups of veterans. Although initial levels of PTSD symptom severity were different across racial/ethnic groups, Caucasian, African American, and Hispanic veterans showed similar rates of improvement. Veterans of varying ages also showed similar rates of improvement as they participated in these therapies. Furthermore, veterans of varying levels of initial PTSD symptom severity all benefited from treatment. These findings suggest that CPT and PE can be applied broadly to the wide population of people in need of treatment for trauma.

The results from the present study also suggest that the VA's national campaign to disseminate CPT and PE has been successful. Mental health practitioners in diverse settings across the United States have been able to learn one or both of these treatments and implement them with the patients under their care. The majority of patients who have received CPT and PE experienced benefits from these treatments. The results suggest that these treatments should continue to be disseminated at the national level.

This study also speaks to the challenges that patients, mental health practitioners, and trainees in the mental health professions encounter in trauma-focused therapy. Dropout from both treatments remains a significant issue. About half of the participants in the present study did not complete a full therapy protocol. It does appear that people who dropout from CPT or PE do report a different trajectory of PTSD symptom reduction than treatment completers. Providers 
may use this information to track outcomes, identify patients who have not reported decreases in their PTSD symptoms, and alter their interventions to reduce the risk of dropout. Many patients who complete CPT or PE may still have clinically significant PTSD symptoms. More could be done to help this group of veterans, who begin therapy with more severe PTSD, achieve a higher level of relief from their symptoms. Finally, trainees who are learning CPT or PE may take heart in the fact that even highly experienced, doctoral-level providers experience challenges related to patient dropout and disengagement from trauma-focused therapy.

\section{Conclusion}

The results from the present study speak to what the research on CPT and PE has repeatedly told us. CPT and PE are both effective treatments that result in a significant reduction in PTSD symptoms over time. Both treatments are equally effective. Furthermore, all veterans who participate in these treatments have the potential to experience significant reductions in PTSD symptoms. Attendance of therapy sessions was the factor that was most related to treatment outcomes. Outcomes across multiple ages were equivalent. Although veterans with higher PCL scores at baseline experienced more significant declines in their PTSD symptoms, veterans of all levels of PTSD symptomology experienced gains during treatment.

Encouragingly, outcomes for Caucasian, African American, and Hispanic veterans were equivalent to one another. Challenges do remain. Dropout remains a significant problem. Furthermore, the final predicted PCL scores for both treatments indicated that a large proportion of veterans who completed either treatment still had clinically significant PTSD symptoms. However, but continued research can help to make both treatments even more effective than they already are. The VA should continue to disseminate CPT and PE across the country so that both treatments are available to the veterans who need them. 
-92- 


\section{References}

Alcántara, C., Casement, M. D., \& Lewis-Fernández, R. (2013). Conditional risk for PTSD among Latinos: a systematic review of racial/ethnic differences and sociocultural explanations. Clin Psychol Rev, 33(1), 107-119. doi: http://dx.doi.org/10.1016/j.cpr.2012.10.005

Alvarez, J., McClean, C., Harris, A., Rosen, C., Ruzek, J., \& Kimerling, R. (2011). The comparitive effectiveness of cognitive processing therapy for male veterans treated in a VHA posttraumatic stress disorder residential rehabilitation program. Journal of Consulting and Clinical Psychology, 79(5), 590-599. doi: http://dx.doi.org/10.1037/a0024466

American Psychological Association (2013). The critical need for mental health professionals trained to treat post-traumatic stress disorder and traumatic brain injury. Retrieved from http://www.apa.org/about/gr/issues/military/critical-need.aspx

Armour, C., Elhai, J. D., Richardson, D., Ractliffe, K., Wang, L., \& Elklit, A. (2012). Assessing a five factor model of PTSD: is dysphoric arousal a unique PTSD construct showing differential relationships with anxiety and depression? J Anxiety Disord, 26(2), 368-376. doi: 10.1016/j.janxdis.2011.12.002

Association, A. P. (2003). Guidelines on Multicultural Education, Training, Research, Practice, and Organizational Change for Psychologists. American Psychologist, 58(5), 377-402. doi: http://dx.doi.org/10.1037/0003-066X.58.5.377

Atkinson, Rick. (2002). An army at dawn: The war in North Africa, 1942-1943. New York: Henry Holt \& Co. 
Babington, A. (1993). A stain on Army medicine. Lancet, 342(8882), 1253-1254. doi: http://dx.doi.org/10.1016/0140-6736(93)92356-X

Benish, S. G., Imel, Z. E., \& Wampold, B. E. (2008). Corrigendum to "The relative efficacy of bona fide psychotherapies for treating post-traumatic stress disorder: A meta-analysis of direct comparisons" [Clinical Psychology Review 28 (2008) 766-75]. Clin Psychol Rev, 28(7), 1281. doi: http://dx.doi.org/10.1016/j.cpr.2008.06.001

Benish, S. G., Imel, Z. E., \& Wampold, B. E. (2008). The relative efficacy of bona fide psychotherapies for treating post-traumatic stress disorder: a meta-analysis of direct comparisons. Clin Psychol Rev, 28(5), 746-758. doi: 10.1016/j.cpr.2007.10.005

Birmes, P., Hatton, L., Brunet, A., \& Schmitt, L. (2003). Early historical literature for posttraumatic symptomatology. Stress and Health: Journal of the International Society for the Investigation of Stress, 19(1), 17-26. doi: http://dx.doi.org/10.1002/smi.952

Boscarino, J. A. (1997). Diseases among men 20 years after exposure to severe stress: implications for clinical research and medical care. Psychosom Med, 59(6), 605-614.

Bradley, R. G., Greene, J., Russ, E. U., Dutra, L., \& Westen, D. (2005). A multidimensional meta-analysis of psychotherapy for PTSD. American Journal of Psychiatry, 162(2), 214227. doi: http://dx.doi.org/10.1176/appi.ajp.162.2.214

Brewin, C. R., Andrews, B., \& Valentine, J. D. (2000). Meta-analysis of risk factors for posttraumatic stress disorder in trauma-exposed adults. Journal of Consulting and Clinical Psychology, 68(5), 748-766. doi: http://dx.doi.org/10.1037//0022-006X.68.5.748

Carkhuff, R. R., \& Pierce, R. (1967). Differential effects of therapist race and social class upon patient depth of self-exploration in the initial clinical interview. Journal of Consulting Psychology, 31(6), 632-634. doi: http://dx.doi.org/10.1037/h0025163 
Caska, C. M., \& Renshaw, K. D. (2013). Personality traits as moderators of the associations between deployment experiences and PTSD symptoms in OEF/OIF service members. Anxiety, Stress \& Coping: An International Journal, 26(1), 36-51. doi: http://dx.doi.org/10.1080/10615806.2011.638053

Chambless, D. L., \& Hollon, S. D. (1998). Defining empirically supported therapies. J Consult Clin Psychol, 66(1), 7-18. doi: http://dx.doi.org/10.1037/0022-006X.66.1.7

Chard, K. M. (2005). An evaluation of cognitive processing therapy for the treatment of posttraumatic stress disorder related to childhood sexual abuse. Journal of Consulting and Clinical Psychology, 73(5), 965-971. doi: http://dx.doi.org/10.1037/0022006X.73.5.965

Chard, K. M., Schumm, J. A., Owens, G. P., \& Cottingham, S. M. (2010). A comparison of OEF and OIF veterans and Vietnam veterans receiving cognitive processing therapy. J Trauma Stress, 23(1), 25-32. doi: 10.1002/jts.20500

Chard, K. M., Ricksecker, E. G., Healy, E. T., Karlin, B. E., \& Resick, P. A. (2012). Dissemination and experience with cognitive processing therapy. Journal of Rehabilitation Research \& Development, 9(5), 667-678. DOI: http://dx.doi.org/10.1682/JRRD.2011.10.0198.

Da Costa, J. M. (1871). On irritable heart; a clinical study of a form of functional cardiac disorder and its consequences. American Journal of the Medical Sciences, 61(121), 1752.

Dao, J. \& Lehren, A.W. (2013, May $15^{\text {th }}$ ). Baffling rise in suicides plagues the U.S. military. New York Times. Retrieved from http://www.nytimes.com/2013/05/16/us/baffling-rise-in$\underline{\text { suicides-plagues-us-military.html?pagewanted=all\&_r=0 }}$ 
De Quervain, D. J. F., Kolassa, I.-T., Ackermann, S., Aerni, A., Boesiger, P., Demougin, P., .. . Papassotiropoulos, A. (2012). PKC alpha is genetically linked to memory capacity in healthy subjects and to risk for posttraumatic stress disorder in genocide survivors. Proceedings of the National Academy of Sciences of the United States of America, 109(22), 8746-8751. doi: http://dx.doi.org/10.1073/pnas.1200857109

Dell'Osso, L., \& Carmassi, C. (2011). PTSD 30 years after DSM-III: Current controversies and future challenges. Giornale Italiano di Psicopatologia / Italian Journal of Psychopathology, 17(1), 1-4.

Eftekhari, A., Ruzek, J. I., Crowley, J. J., Rosen, C. S., Greenbaum, M. A., \& Karlin, B. E. (2013). Effectiveness of national implementation of prolonged exposure therapy in veterans affairs care. JAMA Psychiatry, 70(9), 949-955. DOI: http://dx.doi.org/10.1001/jamapsychiatry.2013.36.

Elhai, J. D., Reeves, A. N., \& Frueh, B. C. (2004). Predictors of Mental Health and Medical Service Use in Veterans Presenting With Combat-Related Posttraumatic Stress Disorder. Psychological Services, 1(2), 111-119. doi: http://dx.doi.org/10.1037/1541-1559.1.2.111

Elkin, I., Falconnier, L., Martinovich, Z., \& Mahoney, C. (2006). Therapist effects in the National Institute of Mental Health Treatment of Depression Collaborative Research Program. Psychotherapy Research, 16(2), 144-160. doi: http://dx.doi.org/10.1080/10503300500268540

Faul, F., Erdfelder, E., Lang, A.-G., \& Buchner, A. (2007). G*Power 3: A flexible statistical power analysis program for the social, behavioral, and biomedical sciences. Behavior Research Methods, 39(2), 175-191. doi: http://dx.doi.org/10.3758/BF03193146 
Feeny, N. C., Zoellner, L. A., Mavissakalian, M. R., \& Roy-Byrne, P. P. (2009). What would you choose? Sertraline or prolonged exposure in community and PTSD treatment seeking women. Depression and Anxiety, 26(8), 724-731. doi: http://dx.doi.org/10.1002/da.20588

Foa, E. B. (2011). Prolonged exposure therapy: past, present, and future. Depress Anxiety, 28(12), 1043-1047. doi: 10.1002/da.20907

Foa, E. B., \& Kozak, M. J. (1986). Emotional processing of fear: exposure to corrective information. Psychol Bull, 99(1), 20-35. doi: http://dx.doi.org/10.1037//00332909.99.1.20

Foa, E. B., Rothbaum, B. O., Riggs, D. S., \& Murdock, T. B. (1991). Treatment of posttraumatic stress disorder in rape victims: a comparison between cognitive-behavioral procedures and counseling. J Consult Clin Psychol, 59(5), 715-723.

Foa, E. B., Hembree, E. A., Cahill, S. P., Rauch, S. A., \& Riggs, D. S. (2005). Randomized trial of prolonged exposure for posttraumatic stress disorder with and without cognitive restructuring: outcome at academic and community clinics. Journal of Consulting and Clinical Psychology, 73(5), 953-964, DOI: http://dx.doi.org/10.1037/0022$\underline{006 X .73 .5 .953}$

Foa, E. B., Zoellner, L. A., Feeny, N. C., Hembree, E. A., \& Alvarez-Conrad, J. (2007)(. Does imaginal exposure exacerbate PTSD symptoms? Journal of Consulting and Clinical Psychology, 70(4), 1022-1028, DOI: http://dx.doi.org.www2.lib.ku.edu:2048/10.1037/0022-006X.70.4.1022.

Forbes, D., Lloyd, D., Nixon, R. D., Elliott, P., Varker, T., Perry, D., . . Creamer, M. (2012). A multisite randomized controlled effectiveness trial of cognitive processing therapy for 
military-related posttraumatic stress disorder. J Anxiety Disord, 26(3), 442-452. doi: 10.1016/j.janxdis.2012.01.006

Frueh, B. C., Elhai, J. D., Monnier, J., Hamner, M. B., \& Knapp, R. G. (2004). Symptom Patterns and Service Use Among African American and Caucasian Veterans With Combat-Related PTSD. Psychological Services, 1(1), 22-30. doi: http://dx.doi.org/10.1037/1541-1559.1.1.22

Frueh, B. C., Gold, P. B., de Arellano, M. A., \& Brady, K. L. (1997). A racial comparison of combat veterans evaluated for PTSD. Journal of Personality Assessment, 68(3), 692-702. doi: http://dx.doi.org/10.1207/s15327752jpa6803_14

Galovski, T. E., Blain, L. M., Mott, J. M., Elwood, L., \& Houle, T. (2012). Manualized therapy for PTSD: Flexing the structure of cognitive processing therapy. Journal of Consulting and Clinical Psychology, 80(6), 968-981.

Gilman, R., Schumm, J. A., \& Chard, K. M. (2012). Hope as a change mechanism in the treatment of posttraumatic stress disorder. Psychological Trauma: Theory, Research, Practice, and Policy, 4(3), 270-277. http://dx.doi.org.www2.lib.ku.edu:2048/10.1037/a0024252.

Goenjian, A. K., Bailey, J. N., Walling, D. P., Steinberg, A. M., Schmidt, D., Dandekar, U., \& Noble, E. P. (2012). Association of TPH1, TPH2, and 5HTTLPR with PTSD and depressive symptoms. Journal of Affective Disorders, 140(3), 244-252. doi: http://dx.doi.org/10.1016/j.jad.2012.02.015

Goodson, J. T., Leftkowitz, C. M., Helstrom, A. W., \& Gawrysiak, M. J., (2013). Outcomes of prolonged exposure therapy for veterans with posttraumatic stress disorder. Journal of Traumatic Stress, 26(4), 419-425. DOI: http://dx.doi.org/10.1002/jts.21830. 
Grieger, T. A., Cozza, S. J., Ursano, R. J., Hoge, C., Martinez, P. E., Engel, C. C., \& Wain, H. J. (2006). Posttraumatic stress disorder and depression in battle-injured soldiers. Am J Psychiatry, 163(10), 1777-1783. doi: http://dx.doi.org/10.1176/appi.ajp.163.10.1777

Grubaugh, A. L., Frueh, B. C., Elhai, J. D., Monnier, J., Knapp, R. G., \& Magruder, K. M. (2006). Racial Differences in Psychiatric Symptom Patterns and Service Use in VA Primary Care Clinics. Psychiatric Services, 57(3), 410-413. doi: http://dx.doi.org/10.1176/appi.ps.57.3.410

Guerra, V. S., \& Calhoun, P. S. (2011). Examining the relation between posttraumatic stress disorder and suicidal ideation in an OEF/OIF veteran sample. J Anxiety Disord, 25(1), 12-18. doi: http://dx.doi.org/10.1016/j.janxdis.2010.06.025

Helms, J. E. (1984). Toward a Theoretical Explanation of the Effects of Race on Counseling: A Black and White Model. Counseling Psychologist, 12(4), 153-165. doi: http://dx.doi.org/10.1177/0011000084124013

Hoexter, M. Q., Fadel, G., Felício, A. C., Calzavara, M. B., Batista, I. R., Reis, M. A., .. . Bressan, R. A. (2012). Higher striatal dopamine transporter density in PTSD: an in vivo SPECT study with TRODAT-1. Psychopharmacology. doi: http://dx.doi.org/10.1007/s00213-012-2755-4

Hoge, C. W., McGurk, D. M., Thomas, J. L., Cox, A. L., Engel, C. C., \& Castro, C. A. (2008). Mild traumatic brain injury in U.S. soldiers returning from Iraq. New England Journal of Medicine, 358(5), 453-463. doi: http://dx.doi.org/10.1056/NEJMoa072972

Hoge, C. W., Terhakopian, A., Castro, C. A., Messer, S. C., \& Engel, C. C. (2007). Association of posttraumatic stress disorder with somatic symptoms, health care visits, and 
absenteeism among Iraq War veterans. American Journal of Psychiatry, 164(1), 150-153. doi: http://dx.doi.org/10.1176/appi.ajp.164.1.150

Hox, J. (2010). Multilevel analysis: Techniques and applications (2 ${ }^{\text {nd }}$ ed.). Mahwah, NJ: Erlbaum.

Institute of Medicine (2013). Returning home from Iraq and Afghanistan: Assessment of readjustment needs of veterans, service members, and their families. National Academy of Sciences. Accessed via http://www.iom.edu/Reports/2013/Returning-Home-from-Iraqand-Afghanistan.aspx.

Jeffreys, M. D., Reinfeld, C., Nair, P. V., Garcia, H. A., Mata-Galan, E., \& Rentz, T. O. (2014). Evaluating treatment of posttraumatic stress disorder with cognitive processing therapy and prolonged exposure in a VHA specialty clinic. Journal of Anxiety Disorders, 28, 108114. http://dx.doi.org/10.1016/j.janxdis.2013.04.010.

Kazdin, A. E. (1998). Research design in clinical psychology (3rd ed.): Allyn \& Bacon, Needham Heights, MA.

Kennedy, J. E., Leal, F. O., Lewis, J. D., Cullen, M. A., \& Amador, R. R. (2010). Posttraumatic stress symptoms in OIF/OEF service members with blast-related and non-blast-related mild TBI. NeuroRehabilitation, 26(3), 223-231.

Kessler, R. C., Sonnega, A., Bromet, E. J., Hughes, M., \& Nelson, C. B. (1995). Posttraumatic stress disorder in the National Comorbidity Survey. Arch Gen Psychiatry, 52(12), 10481060.

Kim, D. M., Wampold, B. E., \& Bolt, D. M. (2006). Therapist effects in psychotherapy: A random-effects modeling of the National Institute of Mental Health treatment of 
depression collaborative research program data. Psychotherapy Research, 16(2), 161172. DOI: http://dx.doi.org/10.1080/10503300500264911.

King, D. W., King, L. A., Gudanowski, D. M., \& Vreven, D. L. (1995). Alternative representations of war zone stressors: relationships to posttraumatic stress disorder in male and female Vietnam veterans. J Abnorm Psychol, 104(1), 184-196. doi: http://dx.doi.org/10.1037//0021-843X.104.1.184

King, L. A., King, D. W., Vogt, D. S., Knight, J., \& Samper, R. E. (2006). Deployment Risk and Resilience Inventory: A Collection of Measures for Studying Deployment-Related Experiences of Military Personnel and Veterans. Military Psychology, 18(2), 89-120. doi: http://dx.doi.org/10.1207/s15327876mp1802_1

Kubany, E. S., Abueg, F. R., Owens, J. A., Brennan, J. M., Kaplan, A. S., \& Watson, S. B. (1995). Initial examination of a multidimensional model of trauma-related guilt: Applications to combat veterans and battered women. Journal of Psychopathology and Behavioral Assessment, 17(4), 353-376. doi: http://dx.doi.org/10.1007/BF02229056

Kulka, R. A., Schlenger, W. E., Fairbank, J. A., Hough, R. L., Jordan, B. K., Marmar, C. R., \& Weiss, D. S. (1990). Trauma and the Vietnam War generation: report of findings from the National Vietnam Veterans Readustment Study: Brunner/Mazel.

Lasiuk, G. C., \& Hegadoren, K. M. (2006a). Posttraumatic stress disorder part I: historical development of the concept. Perspectives in Psychiatric Care, 42(1), 13-20. doi: http://dx.doi.org/10.1111/j.1744-6163.2006.00045.x

Lasiuk, G. C., \& Hegadoren, K. M. (2006b). Posttraumatic stress disorder part II: development of the construct within the North American psychiatric taxonomy. Perspectives in Psychiatric Care, 42(2), 72-81. 
Laska, K. M., Smith, T. L., Wislocki, A. P., Minami, T., \& Wampold, B. E. (2013). Uniformity of evidence-based treatments in practice? Therapist effects in the delivery of cognitive processing therapy for PTSD. Journal of Counseling Psychology, 60(1), 31-41. doi: http://dx.doi.org/10.1037/a0031294

Lee, K. A., Vaillant, G. E., Torrey, W. C., \& Elder, G. H. (1995). A 50-year prospective study of the psychological sequelae of World War II combat. American Journal of Psychiatry, 152(4), 516-522.

Lester, K. M., Resick, P. A., Young-Xu, Y., \& Artz, C. E. (2010). Impact of race on early treatment termination and outcomes in posttraumatic stress disorder treatment. Journal of Consulting and Clinical Psychology, 78(4), 480-489. doi: http://dx.doi.org/10.1037/a0019551

Macdonald, A., Monson, C. M., Doron-Lamarca, S., Resick, P. A., \& Palfai, T. P. (2011). Identifying patterns of symptom change during a randomized controlled trial of cognitive processing therapy for military-related posttraumatic stress disorder. J Trauma Stress, 24(3), 268-276. doi: 10.1002/jts.20642

Magruder, K. M., Frueh, B. C., Knapp, R. G., Davis, L., Hamner, M. B., Martin, R. H., .. . Arana, G. W. (2005). Prevalence of posttraumatic stress disorder in Veterans Affairs primary care clinics. Gen Hosp Psychiatry, 27(3), 169-179. doi: 10.1016/j.genhosppsych.2004.11.001

Magruder, K. M., \& Yeager, D. E. (2009). The prevalence of PTSD across war eras and the effect of deployment on PTSD: a systematic review and meta-analysis. Psychiatric Annals, 39(8), 778-788. doi: http://dx.doi.org/10.3928/00485713-20090728-04 
Marmar, C. R., Weiss, D. S., Schlenger, W. E., Fairbank, J. A., \& et al. (1994). Peritraumatic Dissociation and Posttraumatic Stress in Male Vietnam Theater Veterans. Am J Psychiatry, 151(6), 902-907.

Marshall, G. N., Schell, T. L., \& Miles, J. N. V. (2009). Ethnic differences in posttraumatic distress: Hispanics' symptoms differ in kind and degree. Journal of Consulting and Clinical Psychology, 77(6), 1169-1178. doi: http://dx.doi.org/10.1037/a0017721

Mehta, D., \& Binder, E. B. (2012). Gene x environment vulnerability factors for PTSD: the HPA-axis. Neuropharmacology, 62(2), 654-662. doi: http://dx.doi.org/10.1016/j.neuropharm.2011.03.009

Mental Health Advisory Team V (2008). Accessed via http://www.armymedicine.army.mil/reports/mhat/mhat_v/mhat-v.cfm

Meyers, A.B.R. (1870). On the etiology and prevalence of diseases of the heart among soldiers. London: J. Churchill.

Meyers, C.S. (1915). A contribution to the study of shell shock. Lancet, 188, 316-320.

Milliken, C. S., Auchterlonie, J. L., \& Hoge, C. W. (2007). Longitudinal assessment of mental health problems among active and reserve component soldiers returning from the Iraq War. Journal of the American Medical Association, 298(18), 2141-2148. doi: http://dx.doi.org/10.1001/jama.298.18.2141

Miranda, J., Bernal, G., Lau, A., Kohn, L., Hwang, W.-C., \& LaFromboise, T. (2005). State Of The Science On Psychosocial Interventions For Ethnic Minorities. Annual Review of Clinical Psychology, 1(1), 113-142. doi: http://dx.doi.org/10.1146/annurev.clinpsy.1.102803.143822 
Monson, C. M., Schnurr, P. P., Resick, P. A., Friedman, M. J., Young-Xu, Y., \& Stevens, S. P. (2006). Cognitive processing therapy for veterans with military-related posttraumatic stress disorder. J Consult Clin Psychol, 74(5), 898-907. doi: 10.1037/0022006X.74.5.898

Moos, R. (1990). Coping Responses Inventory Manual. Palo Alto, CA: Stanford University and Department of Veterans Affairs Medical Centers.

Muthén, B. O., \& Satorra, A. (1989). Multilevel aspects of varying parameters in structural models. In R. D. Bock (Ed.), Multilevel analysis of educational data (pp. 87-99). San Diego: Academic Press.

Muthén, B. O. (1991). Multilevel factor analysis of class and student achievement components. Journal of Educational Measurement, 28, 338-354. DOI. http://dx.doi.org/10.1111/j.1745-3984.1991.tb00363.x.

Muthén, B. O. (1994). Multilevel covariance structure analysis. Sociological Methods and Research, 22, 376-398.

Muthén, B. O., \& Satorra, A. (1995). Complex sample data in structural equation modeling. In P. V. Marsden (Ed.), Sociological methodology, 1995 (pp. 267-316). Oxford: Blackwell. DOI: http://dx.doi.org/10.2307/1164513.

Nixon, R. D. (2012). Cognitive processing therapy versus supportive counseling for acute stress disorder following assault: a randomized pilot trial. Behav Ther, 43(4), 825-836. doi: 10.1016/j.beth.2012.05.001

National Center for Posttraumatic Stress Disorder (2014). Using the PTSD Checklist for DSMIV (PCL). U.S. Department of Veterans Affairs. Accessed via: http://www.ptsd.va.gov/professional/pages/assessments/assessment-pdf/PCL-handout.pdf 
Norman, S. B., Means-Christensen, A. J., Craske, M. G., Sherbourne, C. D., Roy-Byrne, P. P., \& Stein, M. B. (2006). Associations between psychological trauma and physical illness in primary care. J Trauma Stress, 19(4), 461-470.

Owens, B. D., Kragh, J. F., Jr., Wenke, J. C., Macaitis, J., Wade, C. E., \& Holcomb, J. B. (2008). Combat wounds in operation Iraqi Freedom and operation Enduring Freedom. J Trauma, 64(2), 295-299. doi: 10.1097/TA.0b013e318163b875

Ozer, E. J., Best, S. R., Lipsey, T. L., \& Weiss, D. S. (2003). Predictors of posttraumatic stress disorder and symptoms in adults: a meta-analysis. Psychol Bull, 129(1), 52-73. doi: http://dx.doi.org/10.1037//0033-2909.129.1.52

Peugh, J. L. (2010). A practical guide to multilevel modeling. Journal of School Psychology, 48(1), 85-112. doi: http://dx.doi.org/10.1016/j.jsp.2009.09.002

Possemato, K., Wade, M., Andersen, J., \& Ouimette, P. (2010). The impact of PTSD, depression, and substance use disorders on disease burden and health care utilization among OEF/OIF veterans. Psychological Trauma: Theory, Research, Practice, and Policy, 2(3), 218-223. doi: http://dx.doi.org/10.1037/a0019236

Powers, M. B., Halpern, J. M., Ferenschak, M. P., Gillihan, S. J., \& Foa, E. B. (2010). A metaanalytic review of prolonged exposure for posttraumatic stress disorder. Clin Psychol Rev, 30(6), 635-641. doi: http://dx.doi.org/10.1016/j.cpr.2010.04.007

Rauch, S. A. M., Defever, E., Favorite, T., Duroe, A., Garrity, C., Martis, B., \& Liberzon, I. (2009). Prolonged Exposure for PTSD in a Veterans Health Administration PTSD Clinic. J Trauma Stress, 22(1), 60-64. doi: http://dx.doi.org/10.1002/jts.20380 
Renshaw, K. D. (2011). An integrated model of risk and protective factors for post-deployment PTSD symptoms in OEF/OIF era combat veterans. Journal of Affective Disorders, 128(3), 321-326. doi: http://dx.doi.org/10.1016/j.jad.2010.07.022

Resick, P. A., Galovski, T. E., Uhlmansiek, M. O. B., Scher, C. D., Clum, G. A., \& Young-Xu, Y. (2008). A randomized clinical trial to dismantle components of cognitive processing therapy for posttraumatic stress disorder in female victims of interpersonal violence. Journal of Consulting and Clinical Psychology, 76(2), 243-258. doi: http://dx.doi.org/10.1037/0022-006X.76.2.243

Resick, P. A., Nishith, P., Weaver, T. L., Astin, M. C., \& Feuer, C. A. (2002). A comparison of cognitive-processing therapy with prolonged exposure and a waiting condition for the treatment of chronic posttraumatic stress disorder in female rape victims. Journal of Consulting and Clinical Psychology, 70(4), 867-879. doi:

http://dx.doi.org/10.1037//0022-006X.70.4.867

Resick, P. A., \& Schnicke, M. K. (1992). Cognitive processing therapy for sexual assault victims. Journal of Consulting and Clinical Psychology, 60(5), 748-756. doi: http://dx.doi.org/10.1037//0022-006X.60.5.748

Rosenheck, R., \& Fontana, A. (1996). Race and outcome of treatment for veterans suffering from PTSD. J Trauma Stress, 9(2), 343-351. doi: http://dx.doi.org/10.1002/jts.2490090215

Rosenheck, R., Fontana, A., \& Cottrol, C. (1995). Effect of clinician-veteran racial pairing in the treatment of posttraumatic stress disorder. Am J Psychiatry, 152(4), 555-563.

Schnurr, P. P., Friedman, M. J., \& Rosenberg, S. D. (1993). Premilitary MMPI scores as predictors of combat-related PTSD symptoms. Am J Psychiatry, 150(3), 479-483. 
Schnurr, P. P., Lunney, C. A., Bovin, M. J., \& Marx, B. P. (2009). Posttraumatic stress disorder and quality of life: extension of findings to veterans of the wars in Iraq and Afghanistan. Clin Psychol Rev, 29(8), 727-735. doi: http://dx.doi.org/10.1016/j.cpr.2009.08.006

Schnurr, P. P., Lunney, C. A., \& Sengupta, A. (2004). Risk factors for the development versus maintenance of posttraumatic stress disorder. J Trauma Stress, 17(2), 85-95. doi: http://dx.doi.org/10.1023/B:JOTS.0000022614.21794.f4

Schnurr, P. P., Lunney, C. A., Sengupta, A., \& Waelde, L. C. (2003). A descriptive analysis of PTSD chronicity in Vietnam veterans. J Trauma Stress, 16(6), 545-553. doi: http://dx.doi.org/10.1023/B:JOTS.0000004077.22408.cf

Schumm, J. A., Walter, K. H., \& Chard, K. M. (2013). Latent class differences explain variability in PTSD symptom changes during cognitive processing therapy for veterans. Psychological Trauma: Theory, Research, Practice, and Policy, 5(6), 536-544. doi: http://dx.doi.org/10.1037/a0030359

Scott, J. C., Pietrzak, R. H., Mattocks, K., Southwick, S. M., Brandt, C., \& Haskell, S. (2013). Gender differences in the correlates of hazardous drinking among Iraq and Afghanistan veterans. Drug and Alcohol Dependence, 127(1-3), 15-22. doi: http://dx.doi.org/10.1016/j.drugalcdep.2012.06.003

Seal, K. H., Metzler, T. J., Gima, K. S., Bertenthal, D., Maguen, S., \& Marmar, C. R. (2009). Trends and risk factors for mental health diagnoses among Iraq and Afghanistan veterans using Department of Veterans Affairs health care, 2002-2008. American Journal of Public Health, 99(9), 1651-1658. doi: http://dx.doi.org/10.2105/AJPH.2008.150284

Shalev, A. Y., Bonne, O. B., \& Eth, S. (1996). Treatment of posttraumatic stress disorder: a review. Psychosom Med, 58(2), 165-182. 
Sharkansky, E. J., King, D. W., King, L. A., Wolfe, J., Erickson, D. J., \& Stokes, L. R. (2000). Coping with Gulf War combat stress: Mediating and moderating effects. J Abnorm Psychol, 109(2), 188-197. doi: http://dx.doi.org/10.1037//0021-843X.109.2.188

Shay, J. (1991). Learning about combat stress from Homer's Iliad. J Trauma Stress, 4(4), 561579. doi: http://dx.doi.org/10.1002/jts.2490040409

Shea, M. T., Vujanovic, A. A., Mansfield, A. K., Sevin, E., \& Liu, F. (2010). Posttraumatic stress disorder symptoms and functional impairment among OEF and OIF National Guard and Reserve veterans. J Trauma Stress, 23(1), 100-107.

Sherman, J. J. (1998). Effects of psychotherapeutic treatments for PTSD: a meta-analysis of controlled clinical trials. J Trauma Stress, 11(3), 413-435. doi: 10.1023/A:1024444410595

Simms, L. J., Watson, D., \& Doebbeling, B. N. (2002). Confirmatory factor analyses of posttraumatic stress symptoms in deployed and nondeployed veterans of the Gulf War. $J$ Abnorm Psychol, 111(4), 637-647. doi: http://dx.doi.org/10.1037/0021-843X.111.4.637

Singer, J. D. \& Willett, J. B. (2003). Applied Longitudinal Data Analysis: Modeling Change and Event Occurrence. Oxford University Press, New York.

Spiro, A., Schnurr, P. P., \& Aldwin, C. M. (1994). Combat-related posttraumatic stress disorder symptoms in older men. Psychology and Aging, 9(1), 17-26. doi: http://dx.doi.org/10.1037//0882-7974.9.1.17

Stecker, T., Shiner, B., Watts, B. V., Jones, M., \& Conner, K. R. (2013). Treatment-Seeking Barriers for Veterans of the Iraq and Afghanistan Conflicts Who Screen Positive for PTSD. Psychiatric services (Washington, D.C.), 64(3), 280-283. 
Sue, S. \& Zane, N. (2006). Ethnic Minority Populations Have Been Neglected by EvidenceBased Practices. In Norcross, J., Beutler, L., \& Levant, R. (Eds.), Evidence-Based Practices In Mental Health (329-337). Washington D.C.: American Psychological Association.

Sundin, J., Fear, N. T., Iversen, A., Rona, R. J., \& Wessely, S. (2010). PTSD after deployment to Iraq: conflicting rates, conflicting claims. Psychol Med, 40(3), 367-382. doi: $10.1017 / \mathrm{S} 0033291709990791$

Tuerk, P. W., Yoder, M., Grubaugh, A. L., Myrick, H., Hamner, M. B., \& Acierno, R. E. (2011). Prolonged exposure therapy for combat-related posttraumatic stress disorder: an examination of treatment effectiveness for veterans of the wars in Afghanistan and Iraq. $J$ Anxiety Disord, 25(3), 397-403. doi: http://dx.doi.org/10.1016/j.janxdis.2010.11.002

U.S. Medicine (2009). VA research proves cognitive behavioral therapy helpful in treating PTSD. U.S. Medicine (November, 2009). Retrieved from http://www.usmedicine.com/articles/va-research-proves-cognitive-behavioral-therapyhelpful-in-treating-ptsd.html\#.UegQvbEo6P8

Van Zuiden, M., Heijnen, C. J., Maas, M., Amarouchi, K., Vermetten, E., Geuze, E., \& Kavelaars, A. (2012). Glucocorticoid sensitivity of leukocytes predicts PTSD, depressive and fatigue symptoms after military deployment: a prospective study. Psychoneuroendocrinology. doi: http://dx.doi.org/10.1016/j.psyneuen.2012.03.018

Vogt, D., Smith, B., Elwy, R., Martin, J., Schultz, M., Drainoni, M.-L., \& Eisen, S. (2011). Predeployment, deployment, and postdeployment risk factors for posttraumatic stress symptomatology in female and male OEF/OIF veterans. J Abnorm Psychol, 120(4), 819831. doi: http://dx.doi.org/10.1037/a0024457 
Walling, S. M., Suvak, M. K., Howard, J. M., Taft, C. T., \& Murphy, C. M. (2012).

Race/ethnicity as a predictor of change in working alliance during cognitive behavioral therapy for intimate partner violence perpetrators. Psychotherapy, 49(2), 180-189. doi: 10.1037/1040-3590.1.3.207.

Wampold, B. E. (2001). The great psychotherapy debate: Models, methods, and findings: Lawrence Erlbaum Associates Publishers, Mahwah, NJ.

Wampold, B. E., Mondin, G. W., Moody, M., Stich, F., \& et al. (1997). A meta-analysis of outcome studies comparing bona fide psychotherapies: Empirically, "all must have prizes". Psychol Bull, 122(3), 203-215.

Wampold, B. E. \& Brown, G. S. (2005). Estimating variability in outcomes attributable to therapists: A naturalistic study of outcomes in managed care. Journal of Consulting and Clinical Psychology, 73(5), 914-923. DOI: $\underline{\text { http://dx.doi.org/10.1037/0022- }}$ $\underline{006 X .73 .5 .914 .}$

Wayman, J. C. (2003). Multiple imputation for missing data: What is it and how can I use it? Paper presented at the 2003 Annual Meeting of the American Educational Research Association, Chicago, IL.

Weathers, F. W., Litz, B., Herman, D., Juska, J., \& Keane, T. (1993). PTSD Checklist-Civilian Version.

Wierzbicki, M., \& Pekarik, G. (1993). A meta-analysis of psychotherapy dropout. Professional Psychology: Research and Practice, 24(2), 190-195. doi: http://dx.doi.org/10.1037/07357028.24.2.190

Yoder, M., Tuerk, P. W., Price, M., Grubaugh, A. L., Strachan, M., Myrick, H., \& Acierno, R. (2012). Prolonged exposure therapy for combat-related posttraumatic stress disorder: 
Comparing outcomes for veterans of different wars. Psychological Services, 9(1), 16-25. doi: http://dx.doi.org/10.1037/a0026279

Zatzick, D. F., Marmar, C. R., Weiss, D. S., Browner, W. S., Metzler, T. J., Golding, J. M., . . . Wells, K. B. (1997). Posttraumatic Stress Disorder and Functioning and Quality of Life Outcomes in a Nationally Representative Sample of Male Vietnam Veterans. Am J Psychiatry, 154(12), 1690-1695.

Zovkic, I. B., \& Sweatt, J. D. (2012). Epigenetic mechanisms in learned fear: implications for PTSD. Neuropsychopharmacology. doi: http://dx.doi.org/10.1038/npp.2012.79 


\section{Appendix A}

\section{Preliminary Analysis Tables}

Table 3. One-way random effect ANOVAs for the three-level and two-level hierarchical models.

\begin{tabular}{lcc}
\hline Parameters & Three-level Model & Two-level Model \\
\hline \multicolumn{3}{c}{ Regression coefficients (fixed effects) } \\
Intercept & $55.43(0.81)^{* * *}$ & $55.90(0.49)^{* * * *}$ \\
& Variance Components (random effects) \\
Level 1 Residual $\left(\sigma_{\text {time }}^{2}\right)$ & $83.55(1.99)^{* * *}$ & $80.80(1.75)^{* * *}$ \\
Level 2 Residual $\left(\sigma_{\text {patient }}^{2}\right)$ & $158.09(10.36)^{* * *}$ & $166.75(9.45)^{* * *}$ \\
Level 3 Residual $\left(\sigma_{\text {therapist }}^{2}\right)$ & $16.68(7.31)^{*}$ & \\
& & \\
& Model Summary & $38,197.88$ \\
Deviance Statistic & $31,590.95$ & $38,203.88$ \\
AIC & $31,596.95$ & $38,223.45$ \\
BIC & $31,615.93$ & 3 \\
Number of estimated parameters & 4 & REML \\
Method of Estimation & REML & \\
\hline
\end{tabular}

Note. $* * * \mathrm{p}<.001 ; * * \mathrm{p}<.01 ; * \mathrm{p}<.05$. 


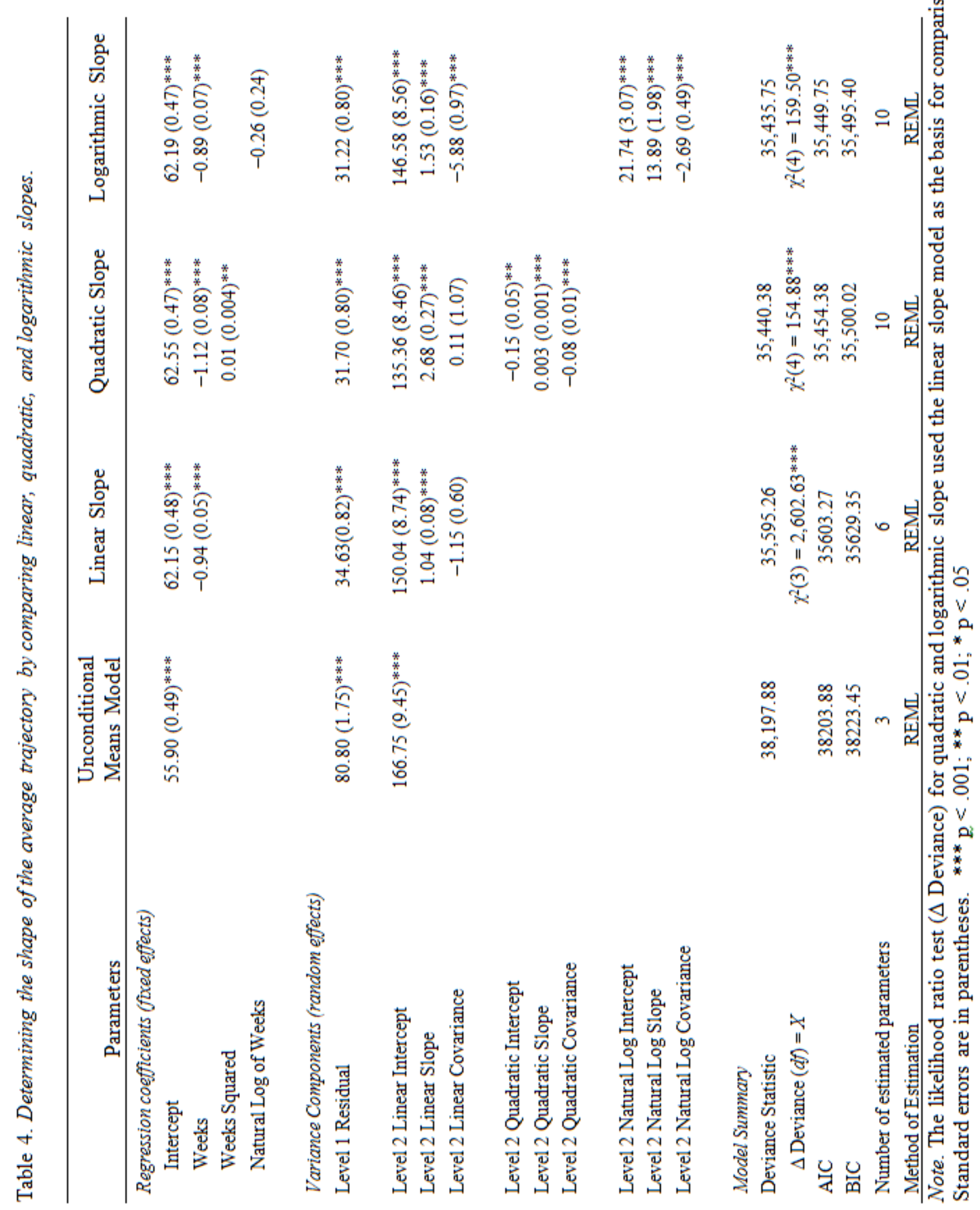


Table 5. Testing for random effects with the linear and quadratic slope terms.

\begin{tabular}{lcc}
\hline Parameters & Quadratic Model 1 & Quadratic Model 2 \\
\hline Regression coefficients (fixed effects) & & \\
$\quad$ Intercept & $61.93(0.48)^{* * *}$ & $61.85(0.49)^{* * *}$ \\
$\quad$ Weeks & $-1.05(0.06)^{* * *}$ & $-0.98(0.06)^{* * *}$ \\
$\quad$ Week Squared & $0.01(0.00)^{* *}$ & $-0.001(0.01)$ \\
\hline Variance Components (random effects) & & \\
Level 1 Residual & $34.66(0.82)^{* * *}$ & $37.670(0.901)^{* * *}$ \\
& & \\
Level 2 Linear Intercept & $149.36(8.60)^{* * *}$ & \\
Level 2 Linear Slope & $1.02(0.08)^{* * *}$ & \\
Level 2 Linear Covariance & $-0.61(0.58)$ & \\
& & $154.26(8.60)^{* * *}$ \\
Level 2 Quadratic Intercept & & $0.004(0.0004)^{* * *}$ \\
Level 2 Quadratic Slope & & $0.09(0.04)^{*}$ \\
Level 2 Quadratic Covariance & & \\
\hline Model Summary & $35,597.68$ & $35,922.22$ \\
Deviance Statistic & $35,605.68$ & $35,930.22$ \\
AIC & $35,631.76$ & $35,956.30$ \\
BIC & 7 & 7 \\
Number of estimated parameters & REML & REML \\
Method of Estimation & & \\
\hline
\end{tabular}

Note. Standard errors are in parentheses. $* * * p<.001 ; * * p<.01 ; * p<.05$. 


\section{Appendix B}

\section{Results From Preliminary Analyses}

The first step in the preliminary analysis was to determine whether MLM was an appropriate statistical procedure for the data. This was achieved by running an unconditional model (a one-way random effects ANOVA), which included no predictors at either level. This model showed the amount of variance in the treatment outcome variable (PCL scores) that was due to each level in the model. The level 1 unit (time) included repeated measures of the PCL that each patient completed while they participated in trauma-focused psychotherapy. Variance in PCL scores due to time is typically a reflection of a patient's participation in psychotherapy, although it may also be due to maturation. Patients were considered to be level 2 units. Variance in PCL scores due to patients is because of individual differences between patients such as differences in trauma history, level of PTSD symptom severity, or level of engagement in the treatment. Results of these analyses are presented in Table 2 in Appendix A.

The intraclass correlation coefficient (ICC) for a level is the percentage of total variance accounted for by that level in the model. The total variance in a two-level hierarchical model is given by

$$
\sigma^{2}=\sigma_{\text {time }}^{2}+\sigma_{\text {patient. }}^{2}
$$

$\sigma^{2}$ time is the amount of variance in PCL scores due to time. Variance in PCL scores due to time is typically a reflection of a patient's participation in psychotherapy, although it may also be due to maturation. $\sigma_{\text {patient }}^{2}$ is the amount of variance in PCL scores due to patients. Variance at this level may be due to individual differences between patients such as trauma history, level of PTSD symptom severity, or level of engagement in the treatment. 
In a two-level hierarchical model, the total variance in PCL scores due to patients can be defined as

$$
\mathrm{ICC}_{\text {patient }}=\sigma_{\text {patient }}^{2} /\left(\sigma_{\text {patient }}^{2}+\sigma_{\text {time }}^{2}\right)
$$

A one-way random effects ANOVA with two levels (level $1=$ time; level 2 = patients) was run with no predictors at either level. The results from the two-level unconditional model showed that $67.3 \%(166.75 /[166.75+80.80]=.673)$ of the variance in PTSD symptoms was due to patient characteristics. The ICC of .67 for patients indicated that MLM would be an appropriate way to analyze the data in this study.

The design effect is a statistic that corrects the ICC for the average number of nested units. The design effect for ICC for patients can be calculated by

$$
\text { Design Effect }=1+\left(n_{\mathrm{c}}-1\right) I C C_{\text {patients }}
$$

where $n_{\mathrm{c}}$ is the average number of repeated measures per patient. The average number of repeated measures per patient $(5,018 / 750=6.69)$ resulted in a large design effect of $4.84(1+$ $[6.69-1] * .674=4.84)$. Some researchers believe that a design effect estimate of greater than 2.00 is needed for MLM (Muthen, 1991, 1994; Muthen \& Satorra, 1989, 1995). Thus, both the ICC for patients and the design effect for patients indicated that MLM would be an appropriate statistical method to analyze the data from this study.

Shape of the slope. The next step in the analysis involved determining the shape of the slope for the model. The results of this analysis are presented in Table 3 in Appendix A. The initial results showed that including a linear slope dramatically improved the quality of the MLM model. The fixed effect for the linear slope term for time was statistically significant $(B=-0.94$, $S E=0.05 p<.001)$. The likelihood ratio test showed that a linear slope was a significant improvement over the unconditional means model, $\chi^{2}(d f=3, N=750)=2,602.63, p<.001$. 
Furthermore, the fit statistics (AIC, BIC) were improved in the model that included a linear slope. Thus, including a linear slope was an improvement over the unconditional model.

Next, a quadratic slope was tested. Results from the quadratic model showed that the quadratic slope term was statistically significant $(B=0.01, S E=0.004, p=.002)$ and that this model explained significantly more variance than the linear slope model, $\chi^{2}(d f=4, N=750)=$ 154.88, $p<.001$. Fit statistics in the quadratic model ( $\mathrm{AIC}=35,603.27$; $\mathrm{BIC}=35,629.35$ ) were lower than in the linear model ( $\mathrm{AIC}=38,203.88 ; \mathrm{BIC}=38,223.45$ ). These findings all suggested that a MLM with a quadratic slope was superior to a MLM with a linear slope.

Next, a logarithmic slope was tested. The fixed effect for the logarithmic slope term was not statistically significant $(B=-0.26, S E=0.24, p=.265)$. At that point, it was concluded that a quadratic slope was the best fit to the data in this study. Thus, all subsequent models assumed the shape of the average slope was quadratic.

Determining Random Effects. The next step of the analysis involved determining whether to allow the linear or quadratic slope term to vary across patients. Results from this analysis are shown in Table 4 in Appendix A.

Quadratic Model 1 included the linear slope term as a random effect, which enabled this value to vary across patients. Quadratic Model 2 kept the linear slope term fixed and allowed the quadratic slope term to vary across patients. All fit statistics were smaller for Quadratic Model 1, which indicated that including the linear slope term as both a fixed and random effect resulted in a superior model. Thus, in all subsequent analyses the linear slope term was included as both a fixed and random effect while the quadratic slope term was included as a fixed effect only. 


\section{Appendix C}

\section{Post-hoc Analysis of Therapist Effects}

An additional question of interest was whether there was enough variance due to therapists to create a three-level hierarchical model. The total variance in a three-level hierarchical model is given by

$$
\sigma^{2}=\sigma_{\text {time }}^{2}+\sigma_{\text {patient }}^{2}+\sigma_{\text {therapist. }}^{2}
$$

$\sigma_{\text {therapist }}^{2}$ is the amount of variance in PCL scores due to therapists.

The ICC for therapists is defined as

$$
\mathrm{ICC}_{\text {therapist }}=\sigma_{\text {therapist }}^{2} /\left(\sigma_{\text {therapist }}^{2}+\sigma_{\text {patient }}^{2}+\sigma_{\text {time }}^{2}\right) .
$$

In the three-level hierarchical model, the overall variance in PCL scores due to therapists was $6.5 \%(\mathrm{ICC}=16.68 /[83.55+158.09+16.68]=.065)$. Although the amount of variance in PCL scores due to therapists was statistically significant at the 0.05 level, $6.5 \%$ is a relatively small amount of variance to account for in subsequent models.

The design effect is a statistic that corrects the ICC for the average number of nested units. The design effect for ICC for therapists can be calculated by

$$
\text { Design Effect }=1+\left(n_{\mathrm{c}}-1\right) I C C_{\text {therapist }}
$$

where $n_{\mathrm{c}}$ is the average number of patients per therapist. The average number of patients per therapists was $11.77(S D=8.50)$. The design effect for therapists was small $(1+[11.77-1] *$ $0.065=1.70)$. Some researchers believe that a design effect estimate of greater than 2.00 is needed for MLM (Muthen, 1991, 1994; Muthen \& Satorra, 1989, 1995). These results indicated that although the amount of variance in PCL scores due to therapists was statistically significant, the structure of the data did not allow for the creation of a three-level hierarchical model. 


\section{Appendix D}

\section{SPSS Multilevel Modeling Syntax}

\section{Preliminary Analyses.}

*Testing for random effects.

mixed rawscore with week_c weekSQ completer completer_int initPTSD_c initPTSD_INT /criteria $=$ mxiter $(1000)$

/fixed=intercept week_c weekSQ completer completer_int initPTSD_c initPTSD_INT

$/$ method=reml

/print=solution testcov

/random=intercept week_c initPTSD_C | subject(patientSID) covtype(un).

\section{* Unconditional means model.}

mixed rawscore

/fixed=intercept

/method=ml

/print=solution testcov

/random=intercept $\mid$ subject(patientSID) covtype(un).

* Unconditional growth model, testing for linear slope.

mixed rawscore with week_c

/fixed=intercept week_c

$/$ method $=\mathrm{ml}$

/print=solution testcov

/random=intercept week_c | subject(patientSID) covtype(un).

*Testing for nonlinear (quadratic) slope.

mixed rawscore with week_c weekSQ

/fixed=intercept week_c weekSQ

/method=ml

/print=solution testcov

/random=intercept week_c $\mid$ subject(patientSID) covtype(un).

*Testing for natural log slope.

mixed rawscore with weeks_c weeksNL

/fixed=intercept weeks_c weeksNL

$/$ method $=\mathrm{ml}$

/print=solution testcov

/random=intercept weeks_c weeksNL | subject(patientSID) covtype(un). 


\section{Primary Analyses}

*Model A.

mixed rawscore with week_c weekSQ completer completer_int completerINT_Quad initPTSD_c initPTSD_INT initPTSDINT_Quad age_c age_INT age_INT_Quad

/criteria $=$ mxiter $(1000)$

/fixed=intercept week_c weekSQ completer completer_int completerINT_Quad initPTSD_c initPTSD_INT initPTSDINT_Quad age_c age_INT age_INT_Quad

/method=ML

/print=solution testcov

/random=intercept week_c $\mid$ subject(patientSID) covtype(un).

*Model B.

mixed rawscore with week_c weekSQ completer completer_int initPTSD_c initPTSD_INT /criteria $=$ mxiter $(1000)$

/fixed=intercept rawscore with week_c weekSQ completer completer_int initPTSD_c initPTSD_INT

/method=ML

/print=solution testcov

/random=intercept week_c $\mid$ subject(patientSID) covtype(un).

*Model C.

mixed rawscore with week_c weekSQ completer completer_int initPTSD_c initPTSD_INT cpt cpt_INT

/criteria $=$ mxiter $(1000)$

/fixed=intercept week_c weekSQ completer completer_int initPTSD_c initPTSD_INT cpt cpt_INT

/method=ML

/print=solution testcov

/random=intercept week_c $\mid$ subject(patientSID) covtype(un).

*Model D.

mixed rawscore with week_c weekSQ completer completer_int initPTSD_c initPTSD_INT cpt cpt_INT cptinT_quad AfAmer AfAmer_INT AfAmer_INT_QUAD hispanic hispanicINT hispanicINT_QUAD

/criteria $=$ mxiter $(1000)$

/fixed=intercept week_c weekSQ completer completer_int initPTSD_c initPTSD_INT cpt cpt_INT cptinT_quad AfAmer AfAmer_INT AfAmer_INT_QUAD hispanic hispanicINT hispanicINT_QUAD

/method=ML

/print=solution testcov

/random=intercept week_c | subject(patientSID) covtype(un). 


\section{Post-hoc Analyses}

*Three level model: An unconditional means model including therapists. mixed rawscore

/fixed=intercept

/method=reml

/print=solution testcov

/random=intercept $\mid$ subject(patientSID*providerid) covtype(un)

/random=intercept $\mid$ subject(providerid) covtype(un).

*Three level model: An unconditional growth model including therapists. mixed rawscore with week_centered

/fixed=intercept week_centered

$/$ method $=\mathrm{ml}$

/print=solution testcov

/random=intercept week_centered | subject(patientSID*providerid) covtype(un)

/random=intercept week_centered | subject(providerid) covtype(un). 


\section{Appendix E}

\section{Number of Sessions Attended}

Table 6. Frequency distribution of number of sessions attended for cognitive processing therapy $(C P T)$ and prolonged exposure (PE).

\begin{tabular}{|c|c|c|}
\hline $\begin{array}{c}\text { Number of } \\
\text { Sessions }\end{array}$ & CPT & PE \\
\hline 1 & 5 & 6 \\
\hline 2 & 17 & 9 \\
\hline 3 & 32 & 33 \\
\hline 4 & 26 & 29 \\
\hline 5 & 22 & 27 \\
\hline 6 & 26 & 28 \\
\hline 7 & 13 & 31 \\
\hline 8 & 25 & 38 \\
\hline 9 & 14 & 37 \\
\hline 10 & 18 & 33 \\
\hline 11 & 29 & 19 \\
\hline 12 & 118 & 28 \\
\hline 13 & 19 & 13 \\
\hline 14 & 3 & 11 \\
\hline 15 & 0 & 4 \\
\hline 16 & 1 & 7 \\
\hline 17 & 3 & 7 \\
\hline 18 & 2 & 3 \\
\hline 19 & 0 & 4 \\
\hline 20 & 0 & 1 \\
\hline 21 & 1 & 2 \\
\hline 22 & 0 & 1 \\
\hline 23 & 0 & 0 \\
\hline 24 & 0 & 1 \\
\hline 25 & 1 & 2 \\
\hline 26 & 0 & 0 \\
\hline 27 & 0 & 0 \\
\hline 28 & 1 & 0 \\
\hline
\end{tabular}

